\title{
AN ELECTROLYTIC METHOD FOR TARTRATE STABILIZATION IN CHARDONNAY WINE
}

\author{
A Thesis \\ presented to \\ the Faculty of California Polytechnic State University, \\ San Luis Obispo
}

In Partial Fulfillment

of the Requirements for the Degree

Master of Science in Agriculture, Specialization in Food Science and Nutrition

by

Michael Chen

July 2016 
(C) 2016

Michael Chen

ALL RIGHTS RESERVED 
TITLE:

AUTHOR:

DATE SUBMITTED:

COMMITTEE CHAIR:

COMMITTEE MEMBER:

COMMITTEE MEMBER:
An Electrolytic Method for Tartrate Stabilization in Chardonnay Wine

Michael Chen

July 2016

Gour Choudhury, Ph.D.

Professor of Food Science

Chad Immoos, Ph.D.

Professor of Chemistry

Stephanie Jung, Ph.D.

Associate Professor of Food Science 


\section{ABSTRACT \\ An Electrolytic Method for Tartrate Stabilization in Chardonnay Wine \\ Michael Chen}

Tartrate stabilization is the process that removes components that contribute to the crystallization of potassium hydrogen tartrate (KHT) and calcium tartrate (CaT) which is an undesirable outcome for wine quality. There are a variety of current tartrate stabilization techniques such as cold stabilization, chemical additives, ion exchange resins, and electrodialysis that stabilize wine, but the most popular being cold stabilization. Cold stabilization requires high amounts of energy and resources to stabilize wine. With the ever increasing demand for more efficient processing, an alternative tartrate stabilization technology based on an electrolytic method was developed and its viability to stabilize wine was determined. Twelve treatments involving different combinations of time and current were replicated three times each on different batches of Chardonnay wine. Several different variables were analyzed for stability and quality purposes. Tartaric acid, potassium, calcium, and conductivity differences were the most important factors for tartrate stability. Temperature, titratable acidity, $\mathrm{pH}$, color (hue and intensity), and chemical oxygen demand (COD) were indicators of sensory quality characteristics of the wine. The concentrations of potassium, calcium, and tartaric acid were reduced by the electrolytic method at satisfactory process parameters, inherently making the wine more stable. The temperature and hue were significantly affected by the electrolytic method and accelerated the oxidative browning process. Electrolytic treatment of Chardonnay is a viable alternative stabilization technology. The technology can be further developed to become a great option in terms of water and energy consumption, process time, and price.

Keywords: Tartrate stabilization, potassium hydrogen tartrate, electrolytic method, cold stabilization 


\section{ACKNOWLEDGMENTS}

I would first and foremost want to thank my graduate committee, especially my advisor, Dr. Gour Choudhury, for his guidance and knowledge. Thanks to the other committee members, Dr. Chad Immoos and Dr. Stephanie Jung, for their advice and constructive feedback. I also want to thank Craig Stubler for taking the time and effort to run ICP samples for me. Another acknowledgement to Dwayne Jones for providing me with equipment and materials needed. I would also like to thank Dr. John Crandall for his discussions and knowledge of enology. I also appreciate the assistance provided by Pam Montalban. I want to thank Melissa Duggan from Cellar 360 for providing the wine samples and consultation needed for this study. Lastly, I would like to acknowledge California State University Agricultural Research Institute for their funding of the project. 


\section{TABLE OF CONTENTS}

Page

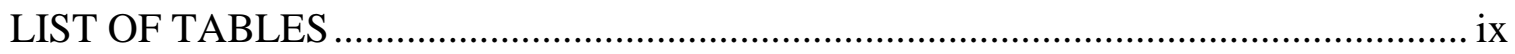

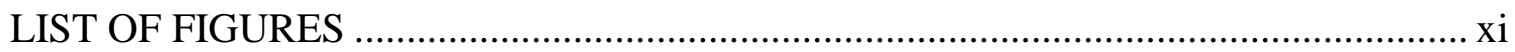

\section{CHAPTER}

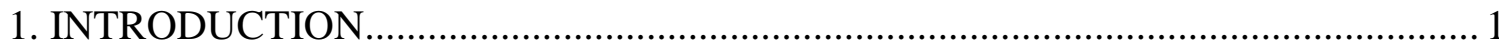

1.1 Goals and Objectives ................................................................................ 2

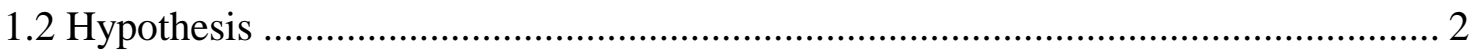

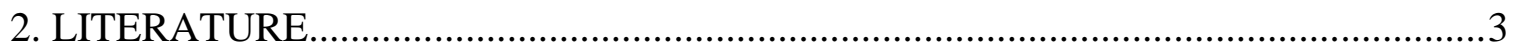

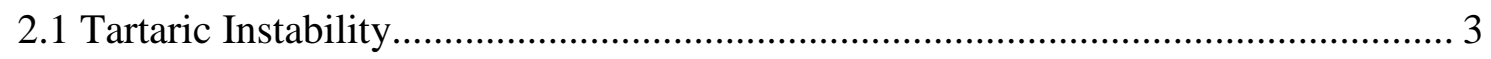

2.1.1 The Effects of Tartaric Acid in Wine ......................................................... 3

2.1.2 Factors Affecting Solubility of Tartrates.............................................. 3

2.2 Determination of Stability of Wine ............................................................. 5

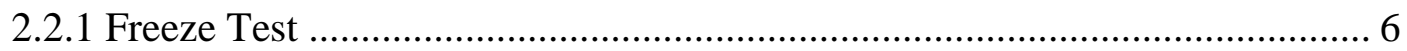

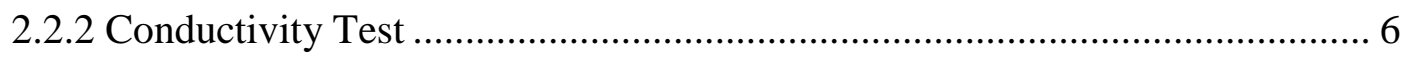

2.2.3 Saturation Temperature Determination ................................................. 7

2.3 Cold Stabilization of Wine ....................................................................... 7

2.3.1 Cold Stabilization Process .................................................................. 7

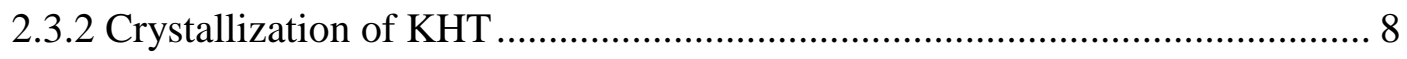

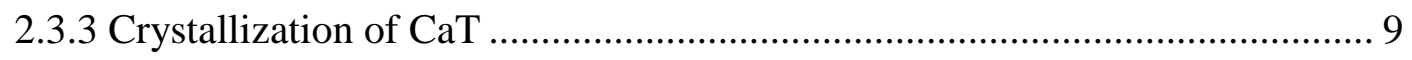

2.3.4 Different Process Variations of Cold Stabilization .................................... 9 
2.3.5 Issues with Cold Stabilization 11

2.4 Alternative Stabilization Technology ........................................................... 12

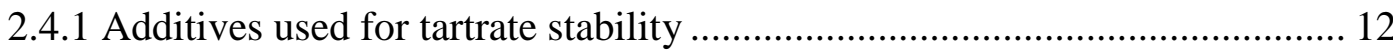

2.4.1.1 Metatartaric acid (MTA) ........................................................ 13

2.4.1.2 Carboxymethycellulose (CMC) ............................................... 13

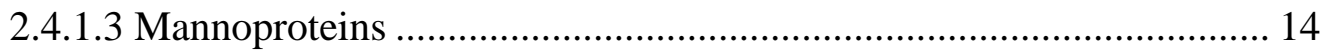

2.4.2 Ion Exchange Resin Process for Wine Stabilization ............................... 15

2.4.3 Electrodialysis Process for Wine Stabilization .................................... 17

2.4.3.1 Energy, Water, and Waste Management...................................... 18

2.4.3.2 Deionization Degree and Stability ............................................. 19

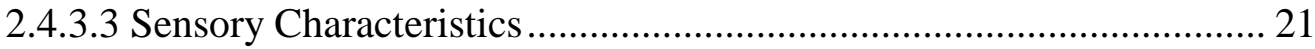

2.5 Economics of Various Wine Stabilization Technologies ................................... 21

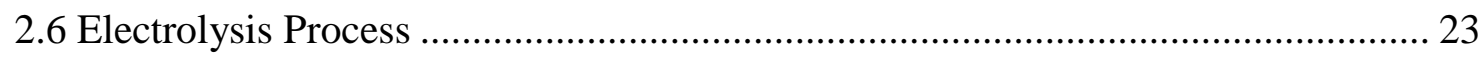

2.6.1 Potential Application of Electrolysis for Wine Stabilization ...................... 24

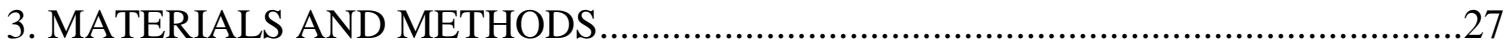

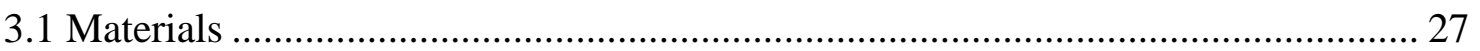

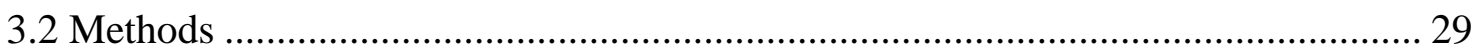

3.2.1 Electrolytic Process ............................................................................. 29

3.2.2 Preliminary Experimental Process ........................................................ 30

3.2.3 Untreated and Treated Wine Analysis................................................ 31 


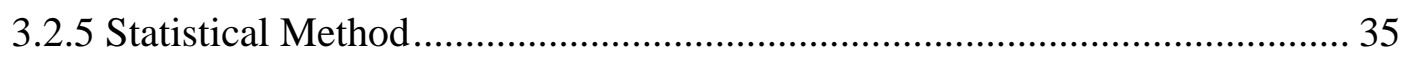

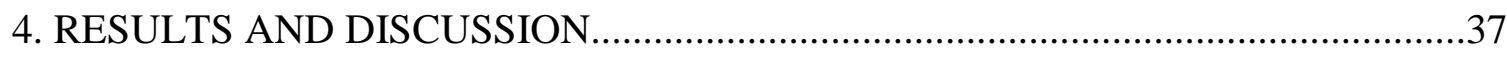

4.1 Determination of Analysis for the Electrolytic Process..................................... 37

4.2 Effect of Electrolytic Process on Temperature Rise of Wine ................................ 39

4.3 Effect of Electrolytic Process on Tartaric Acid Reduction in Wine ...................... 41

4.4 Effect of the Electrolytic Process on Titratable Acidity Concentration in Wine..... 44

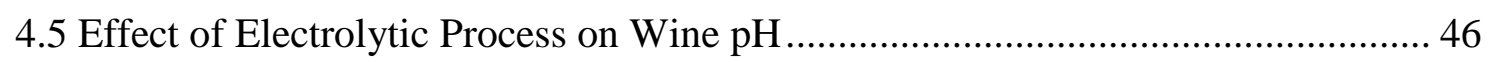

4.6 Effect of Electrolytic Process on the Color of Wine ......................................... 49

4.6.1 Effect of Electrolytic Process on the Hue Value of Wine .................... 49

4.6.2 Effect of Electrolytic Process on the Intensity Value of Wine ............. 51

4.7 Effect of the Electrolytic Process on Mineral (K, Ca, Mg, Na) Reduction in

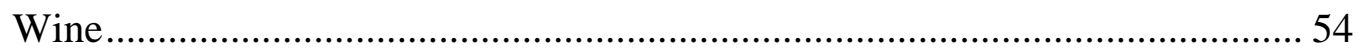

4.8 Comparing Stability Percent Differences of Initial Untreated Wine to Electrolytic Wine 60

4.9 Effect of Electrolytic Process on Chemical Oxygen Demand (COD) Reduction ... 62

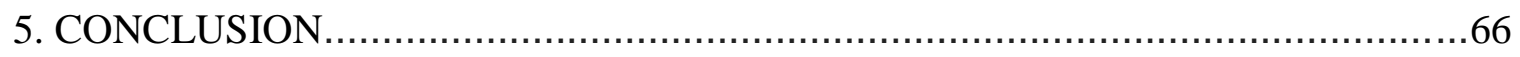

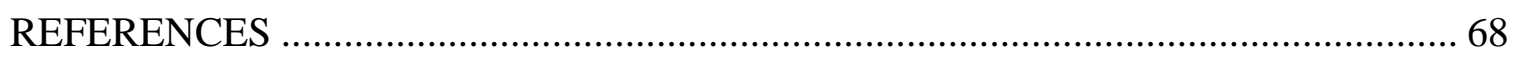

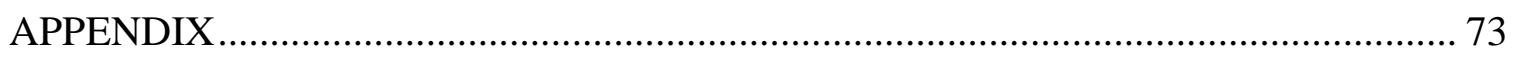




\section{LIST OF TABLES}

Table

Page

Table 1. Comparison of water and energy usage for cold stabilization and electrodialysis 19

Table 2. Cost of stabilizing a liter of wine for various stabilization technologies. 21

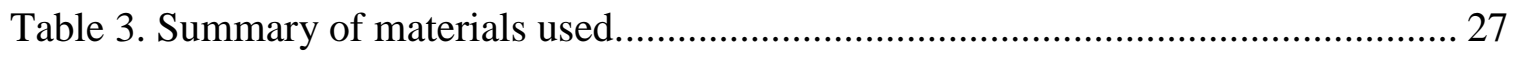

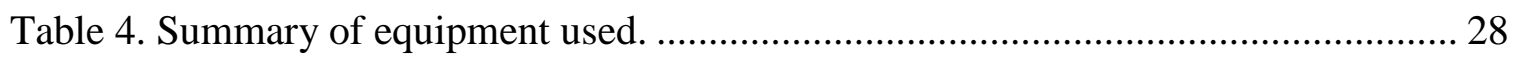

Table 5. Experimental parameters: treatment number, time, current, and charge values..31

Table 6. Tukey comparison of charge treatments for mean differences in temperature... 41

Table 7. Tukey comparison of charge treatments for mean differences in tartaric acid concentration

Table 8. Tukey comparisons of charge treatments for mean differences in titratable acidity. 46

Table 9. Tukey comparison of charge treatments for mean differences in $\mathrm{pH}$................ 48

Table 10. Tukey comparison of charge treatments for mean differences in hue............. 50

Table 11. Tukey comparison of charge treatments for mean differences in intensity...... 52

Table 12. Tukey comparison of charge treatments for mean differences in potassium ... 60

Table 13. Tukey comparison of charge treatments for mean difference in calcium......... 60

Table 14. Tukey comparison of charge treatments for mean differences in magnesium.. 60

Table 15. Tukey comparison of charge treatments for mean differences in sodium ........ 60

Table 16. Tukey comparison of charge treatments for mean differences in COD.......... 63

Table 17. Tukey comparison of treatments for mean differences in temperature............ 73 
Table 18. Tukey comparison of treatments for mean differences in tartaric acid

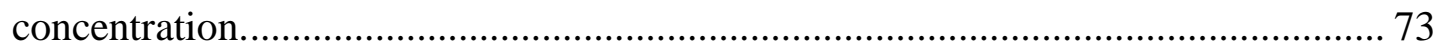

Table 19. Tukey comparison of treatments for least square mean differences in titratable acidity. 73

Table 20. Tukey comparison of treatments for mean differences in $\mathrm{pH}$. ...................... 73

Table 21. Tukey comparison of treatments for mean differences in hue......................... 73

Table 22. Tukey comparison of treatments for mean differences in intensity................ 73

Table 23. Tukey comparison of treatments for mean differences in potassium ............... 74

Table 24. Tukey comparison of treatments for mean difference in calcium .................. 74

Table 25. Tukey comparison of treatments for mean differences in magnesium ............ 74

Table 26. Tukey comparison of treatments for mean differences in sodium................... 74 


\section{LIST OF FIGURES}

Figure $\quad$ Page

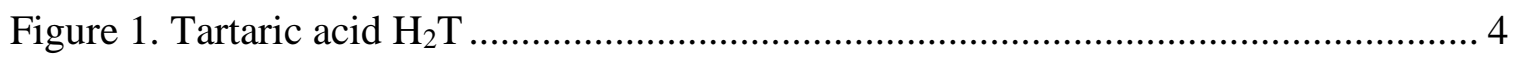

Figure 2. Potassium bitartrate KHT ….............................................................. 4

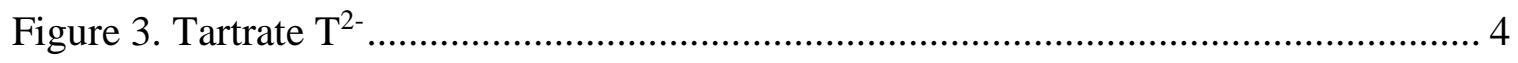

Figure 4. The ratio of tartaric acid, bitartrate, and tartrate concentration with varying $\mathrm{pH}$

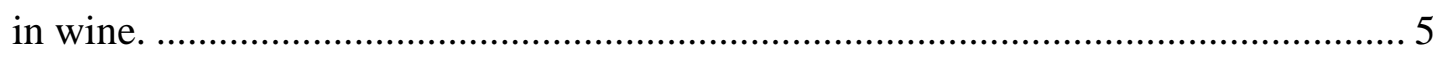

Figure 5. Tartrate crystal formation in wine due to instability. ..................................... 8

Figure 6. Continuous contact system stabilization process. .................................... 10

Figure 7. Metatartaric acid structure, not in polymer form....................................... 13

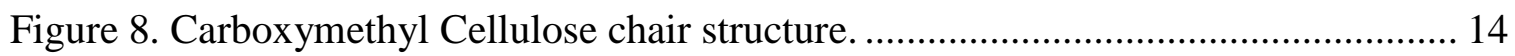

Figure 9. Diagram of a cationic exchanger used to stabilize wine by exchanging

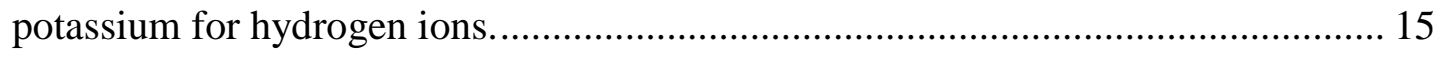

Figure 10. Electrodialysis system diagram for wine stabilization. A is the anionic

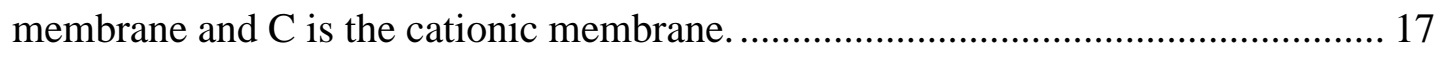

Figure 11. Conventional electrolyzer configuration of water.................................... 23

Figure 12. Attracting of cationic species with a cathode electrode. ............................. 25

Figure 13. Repulsion of cationic species by changing the polarity of the electrodes....... 25

Figure 14. Experimental set-up for electrolysis treatment of wine ............................. 29

Figure 15. Flowchart of the overall experimental process and variables analyzed. ......... 36

Figure 16. Comparison of the effect of electrical charge (coulombs) on the intial and final temperatures for before and after electrolytic treatment at one standard deviation. 
Figure 17. The effect of electrical charge (coulombs) on the temperature differences between the electrolytically treated and initial wines........................................ 40

Figure 18. $\Delta$ Temperature least square means for different charges with $99 \%$ confidence intervals. 41

Figure 19. Comparison of the effect of electrical charge (coulombs) on the intial and final tartaric acid concentration for before and after electrolytic treatment at one standard deviation.

Figure 20. The effect of electrical charge (coulombs) on tartaric acid concentration differences between the initial and electrolytically treated wine.

Figure 21. $\Delta$ Tartaric acid least square means for different charge treatments with $99 \%$ confidence interval. 43

Figure 22. Comparison of the effect of electrical charge (coulombs) on the initial and final titratable acidity concentration for before and after electrolytic treatment at one standard deviation

Figure 23. The effect of electrical charge (coulombs) on titratable acidity concentration differences between the initial and electrolytically treated wine. 45

Figure 24. $\Delta$ Titratable acidity least square means for different charge treatments with $99 \%$ confidence interval. 46

Figure 25. Comparison of the effect of electrical charge (coulombs) on the intial and final $\mathrm{pH}$ for before and after electrolytic treatment at one standard deviation.

Figure 26. The effect of electrical charge (coulombs) on $\mathrm{pH}$ differences between the initial and electrolytically treated wine. 48 
Figure 27. $\Delta \mathrm{pH}$ least square means for different charge treatments with $99 \%$

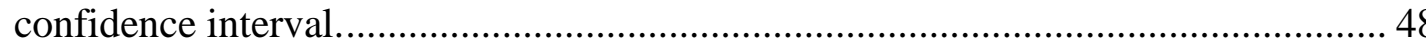

Figure 28. Comparison of the effect of electrical charge (coulombs) on the intial hue for before and after electrolytic treatment at one standard deviation.

Figure 29. The effect of electrical charge (coulombs) on hue differences between the initial and electrolytic treated wine. 50

Figure $30 . \Delta$ Hue means for different charge treatments with $99 \%$ confidence interval... 50

Figure 31. Comparison of the effect of electrical charge (coulombs) on the intial and final Intensity for before and after electrolytic treatment at one standard deviation..51

Figure 32. The effect of electrical charge (coulombs) on intensity differences between the initial and electrolytic treated wine. 52

Figure 33. $\Delta$ Intensity least square means for different charge treatments with $99 \%$ confidence interval. 52

Figure 34. Observational color changes of the wine before (right) and after (left) electrolytic treatment

Figure 35. Comparison of the effect of electrical charge (coulombs) on the initial and final potassium concentration for before and after electrolytic treatment at one standard deviation.

Figure 36. Comparison of the effect of electrical charge (coulombs) on the initial and final calcium concentration for before and after electrolytic treatment at one standard deviation 
Figure 37. Comparison of the effect of electrical charge (coulombs) on the intial and final magnesium concentration for before and after electrolytic treatment at one standard deviation.

Figure 38. Comparison of the effect of electrical charge (coulombs) on the intial and final sodium concentration for before and after electrolytic treatment at one standard deviation. 57

Figure 39. The effect of electrical charge (coulombs) on $\mathrm{K}$ differences between the initial and electrolytically treated wine. 58

Figure 40. The effect of electrical charge (coulombs) on Ca differences between the initial and electrolytically treated wine. 58

Figure 41. The effect of electrical charge (coulombs) on Mg differences between the initial and electrolytically treated wine. 58

Figure 42. The effect of electrical charge (coulombs) on Na differences between the initial and electrolytically treated wine. 58

Figure 43. $\Delta \mathrm{K}$ means for different charge treatments with $99 \%$ confidence interval....... 59

Figure 44. $\Delta$ Ca means for different charge treatments with $99 \%$ confidence interval..... 59

Figure $45 . \Delta \mathrm{Mg}$ means for different charge treatments with $99 \%$ confidence interval.... 59

Figure 46. $\triangle \mathrm{Na}$ means for different charge treatments with $99 \%$ confidence interval..... 59

Figure 47. Average initial conductivity percent differences and the average final conductivity percent differences for all treatments within one standard deviation compared with the $5 \%$ difference stability standard.

Figure 48. The effect of electrical charge (coulombs) on COD differences between the initial and electrolytic treated wine. 63 
Figure 49. $\triangle$ COD means for different charge treatments with $99 \%$ confidence interval.. 63

Figure 50. Scaling on cathode for $0.3 \mathrm{C}$ 65

Figure 51. Scaling on cathode for $0.6 \mathrm{C}$ 65 


\section{CHAPTER 1}

\section{INTRODUCTION}

Water and non-renewable energy are important resources that are becoming more limited due to sustainability issues (MacDonald 2010). In California, a significant drought started in 2015 where the amount of available water is diminishing and raising the price of water. Therefore, it is more important now than ever, to save resources wherever possible. Vineyards and the process of making wine are highly affected by limited water resources. More specifically, many of the technologies used to stabilize wine require energy and water intensive equipment. Water usage can reach 0.2 liter of water per liter of wine and energy usage can reach $17 \mathrm{Wh}$ per liter of wine(Low et al. 2008).

Tartrate stabilization is a process used to remove components that contribute to the crystallization of potassium hydrogen tartrate (KHT) in finished wine. KHT crystals form in finished bottles of wine when there is tartrate instability and this can affect the quality and marketability of the product. The most common way KHT is removed is by the cold stabilization method, where at least $90 \%$ of commercial wineries use this method (Duggan 2015). Cold stabilization is done by lowering the temperature of the wine to freezing temperatures for several days in order to lower the solubility of KHT and induce crystallization (Zoecklein et al. 1990). Another increasingly common method is to use additives like carboxymethyl cellulose (CMC) or mannoproteins. These additives reduce the crystallization formation interactions after mixing with the wine for a couple days at around $17^{\circ} \mathrm{C}$ (Ribereau-Gayon et al. 2006).

More rapid methods of stabilization are ion exchange and electrodialysis. Ion exchange uses a cation specific resin to remove components affecting KHT 
crystallization after passing through a column once (Benítez et al. 2002). Electrodialysis uses an applied electric potential and ion exchange membranes to separate out KHT components from the wine in one pass (Soares et al. 2009).

Even with a multitude of stabilization methods, not one is clearly better than the others in terms of processing time, sensory evaluations, energy usage, water consumption, and price (Low et al. 2008). In times of depleting water and energy resources, it is imperative that a more environmentally conscious and economic stabilization method be developed. In our study, an electrolytic technology is developed to address some of these concerns.

\subsection{Goals and Objectives}

The main goal of this study was to develop an alternative wine stabilization technology for tartaric acid that has low chemical inputs relative to cold stabilization. A secondary goal was to develop an alternative wine stabilization technology that requires less energy and water resources than cold stabilization technology. The objectives included determining how effective an electrolytic method was in stabilizing the wine with current and process time as the two main factors. Differences in $\mathrm{K}, \mathrm{Ca}, \mathrm{Mg}, \mathrm{Na}$, tartaric acid, $\mathrm{pH}$, titratable acidity, conductivity, color, and chemical oxygen demand (COD) were also determined and analyzed for their impacts on tartrate stabilization.

\subsection{Hypothesis}

The electrolytic process developed would provide tartrate stabilization with current and processing time being the most influential. As processing time and current increase, all response variables would decrease accordingly except color. 


\section{CHAPTER 2}

\section{LITERATURE REVIEW}

\subsection{Tartaric Instability}

\subsubsection{The Effects of Tartaric Acid in Wine}

Tartaric acid $\left(\mathrm{H}_{2} \mathrm{~T}\right)$, potassium bitartrate $(\mathrm{KHT})$, and calcium tartrate $(\mathrm{CaT})$ are all natural components in wine and are the main constituents that contribute the most to the total acidity of the wine (Figures 1,2 and 3). The concentration of tartaric acid in grapes varies depending on variety, maturity, and region (Zoecklein et al. 1990). The average KHT and CaT concentrations in wine are typically higher than its solubility in wine, a super saturated solution in wine forms naturally occurring tartrate crystal deposits in bottles, which are then considered to be tartrate unstable (Zoecklein et al. 1990). These crystals formed are considered defects by consumers, even though they are not hazardous and do not affect the flavor (Ribereau-Gayon et al. 2006). Because of a lack of consumer acceptance and marketability, wineries elect to remove components that contribute to the formation of tartrate crystals from the wine before bottling. The formation of tartrate crystals is not only influenced by the concentration of KHT and CaT but by other properties such as the wine's composition, $\mathrm{pH}$, and temperature.

\subsubsection{Factors Affecting Solubility of Tartrates}

The solubility of $\mathrm{H}_{2} \mathrm{~T}$, KHT, and $\mathrm{CaT}$ in water at $20^{\circ} \mathrm{C}$ are $4.9 \mathrm{~g} / 1,5.7 \mathrm{~g} / 1$, and $0.53 \mathrm{~g} / \mathrm{l}$ (Figure 1, 2, 3). They are relatively soluble in water, except calcium tartrate. However, in wine which has at least $10 \% \mathrm{v} / \mathrm{v}$ alcohol, the solubility of all tartaric salts drop. KHT drops to $2.9 \mathrm{~g} / \mathrm{l}$. Also, the $\mathrm{pKa}_{1}$ of tartaric acid is 3.04 and the $\mathrm{pKa}_{2}$ is 4.37 (Zoecklein et al. 1990). Therefore, the $\mathrm{pH}$ of the wine also affects the composition and concentration of tartaric salt. 


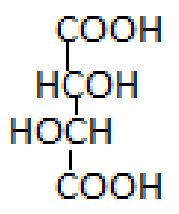

Figure 1. Tartaric acid $\mathrm{H}_{2} \mathrm{~T}$

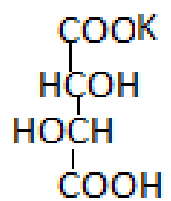

Figure 2. Potassium bitartrate KHT

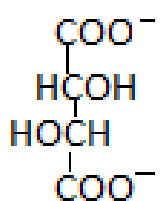

Figure 3. Tartrate $\mathbf{T}^{2-}$

Alcohol content, temperature, and $\mathrm{pH}$ all play a big role in tartrate salt solubility (O'Brien 2014). If the KHT is not soluble or the solution becomes supersaturated, the crystals will form and make the wine unstable. KHT is soluble in water, but relatively insoluble in alcohol (Ribereau-Gayon et al. 2006). For example, in a 10\% v/v alcohol solution at $20{ }^{\circ} \mathrm{C}$, the solubility of KHT is $2.9 \mathrm{~g} / \mathrm{L}$, however, KHT concentrations in wine on average can be around $3.76 \mathrm{~g} / \mathrm{L}$. Therefore, normally the KHT concentration in wine being relatively insoluble in alcohol, causes the wine to become supersaturated.

In addition, when temperatures are lowered, the solubility of KHT decreases (Zoecklein et al. 1990). When wine is supersaturated with KHT and becomes increasingly insoluble due to alcohol content and decreased temperatures, precipitation occurs. First the induction stage happens, where the concentration of KHT nuclei increase spontaneously but slowly (Ribereau-Gayon et al. 2006). Then the crystallization stage, where crystal growth and development occur (Zoecklein et al. 1990). The precipitation rate is fairly rapid in the first 12 days, but decreases due to lower concentration of KHT. Zoecklein et al. (1990) found that the optimal temperature required for KHT stabilization to occur is determined by Temperature $\left(-{ }^{\circ} \mathrm{C}\right)=\frac{\text { alcohol\% }}{2}-1$.

In addition to alcohol percentage and temperature affecting the stabilization of wine, $\mathrm{pH}$ contributes as well. The ratio between tartaric acid, bitartrate, and tartrate are dependent on the $\mathrm{pH}$ (Figure 4). With a $\mathrm{pH}$ of less than 3.0, tartaric acid is of the highest 
concentration because it is below the pKa1 3.04. However, with a $\mathrm{pH}$ between 3.0 and 4.5, KHT is of the highest concentration with a maximum at 3.7. And with a $\mathrm{pH}$ of greater than 4.5, CaT is of the highest concentration because it is above the pKa2 of 4.37 . By knowing what the $\mathrm{pH}$ and $\mathrm{pKa}$ of tartaric acid is, it is possible to ascertain which composition of tartaric acid is the highest. This gives insight into the likelihood of whether or not the wine will undergo tartaric instability for KHT or CaT.

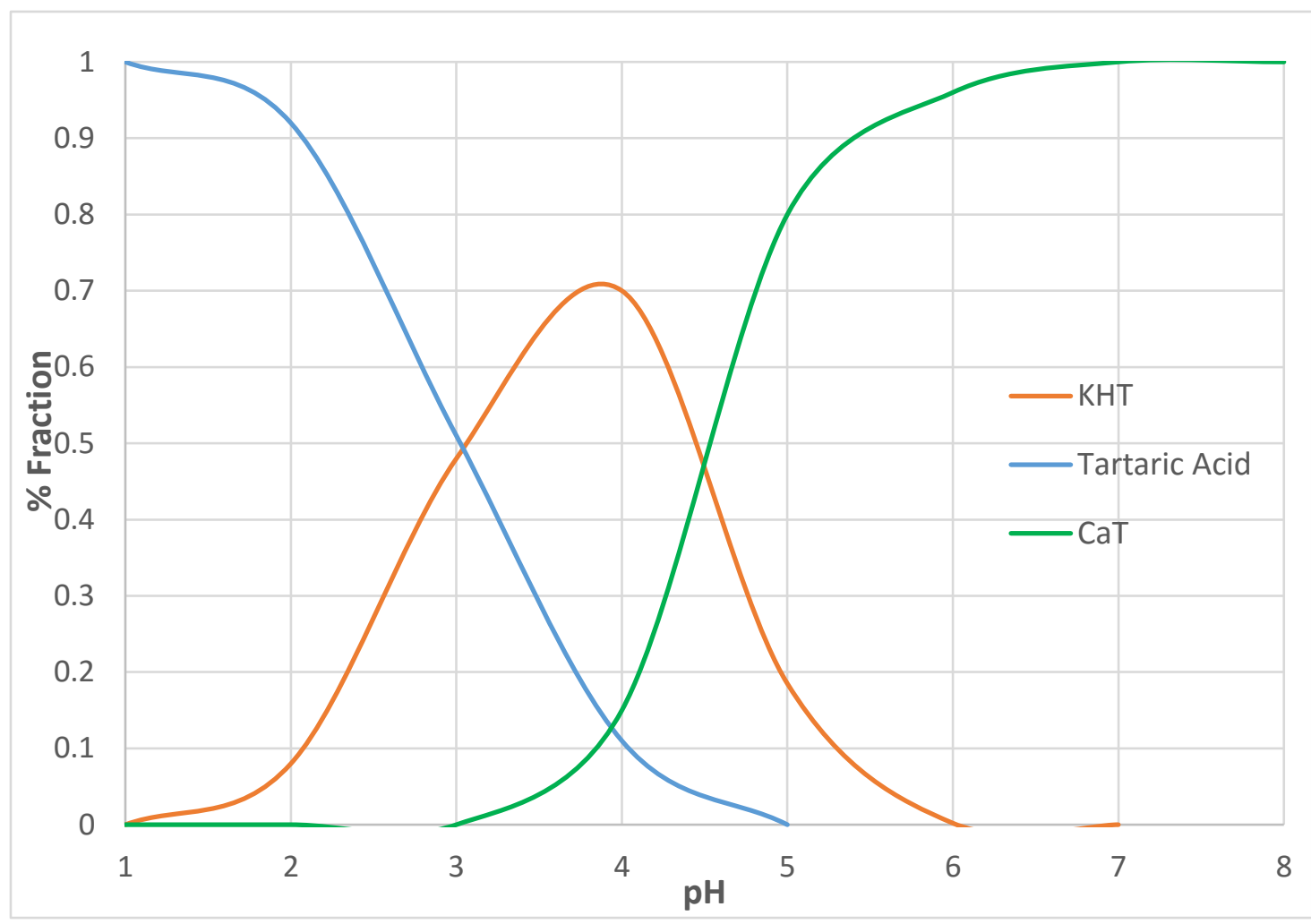

Figure 4. The ratio of tartaric acid, bitartrate, and tartrate concentration with varying pH in wine. Adapted from Zoecklein et al. 1990.

\subsection{Determination of Stability of Wine}

The stability of wine can be determined by several methods; the freezing test, conductivity test, and saturation temperature determination. 


\subsubsection{Freeze Test}

The freeze test takes advantage of the decrease in solubility of KHT at low temperatures. A wine is considered unstable if any KHT crystals form. There is no agreed upon conditions for the freeze test, but generally $100 \mathrm{~mL}$ or less wine sample is placed in a freezer at $0^{\circ} \mathrm{C}$ for 72 hours then thawed and observed for any crystallization. However, some use a membrane filter beforehand or freeze for longer times, and these different treatment conditions have been shown to produce different results. Therefore, the freeze test while the simplest, is the least reliable because it depends on spontaneous noninduced crystallization (Zoecklein et al. 1990; Ribereau-Gayon et al. 2006)

\subsubsection{Conductivity Test}

The conductivity test measures the conductivity changes in the wine to determine if the wine is stable. The main conducting species in wine is potassium $(\mathrm{K})$, which is the major component that contributes to KHT formation. Fine KHT crystals at $10 \mathrm{~g} / \mathrm{L}$ are added to the wine sample to induce crystallization at low temperatures like $0^{\circ} \mathrm{C}$. If the wine is stable, there will be a lack of crystal formation, meaning the $\mathrm{K}$ will not form KHT. A stable wine will have a change of less than $5 \%$ in conductivity value between the original value and the value after KHT crystals are added. If the wine is unstable, the KHT crystals added will induce crystallization of the wine and lower the conductivity, because there is less free $\mathrm{K}$ in the wine solution. An unstable wine will have a change of more than $5 \%$ in its final conductivity compared to the original conductivity. This test is much faster and more reliable than the freeze test. However, it still lacks reliability because the results can change depending on the size and amount of the KHT crystals added (Zoecklein et al. 1990; Ribereau-Gayon et al. 2006) 


\subsubsection{Saturation Temperature Determination}

The saturation temperature of the wine is the lowest temperature at which the wine is capable of dissolving KHT. Knowing the saturation temperature can give insight into what temperatures the wine will be stable up to. The equation $\mathrm{T}_{\text {(stable })}=\mathrm{T}_{\text {sat }}-15^{\circ} \mathrm{C}$ is used to determine what temperature the wine will be stable at. The lower the saturation temperature, the more stable the wine is. The saturation temperature is determined by measuring the conductivity of the wine continuously as the temperature is raised from $0^{\circ} \mathrm{C}$ to $20^{\circ} \mathrm{C}$ at a rate of $0.5^{\circ} \mathrm{C} / \mathrm{min}$. The same procedure is done for the wine containing 4 $\mathrm{g} / \mathrm{L}$ of KHT. The two graphs of conductivity versus temperature are superimposed and the point of intersection of the two lines is the saturation temperature. The determination of saturation temperature of the wine is the most accurate and reliable compared to the other tests, because it measures the solubility of the salt, and not the crystallization rate, which is more unpredictable (Gonçalves et al. 2003; Ribereau-Gayon et al. 2006)

\subsection{Cold Stabilization of Wine}

\subsubsection{Cold Stabilization Process}

Cold Stabilization is a technological method to stabilize wine by removing KHT from wine and is done by cooling the wine in a vessel to about the freezing point for usually a week (Lasanta and Gómez 2012). The vessels are made of stainless steel holding thousands of gallons and by using a combination of heat exchangers, insulators, and cooling units, these vessels are constantly kept at temperatures between -4 and $0{ }^{\circ} \mathrm{C}$. As noted before, the reason for doing this is to lower the solubility of KHT in wine so it crystallizes (Figure 5). Once the wine becomes stable after sufficient crystallization, the wine and crystals are separated by filtering through diatomaceous earth. 


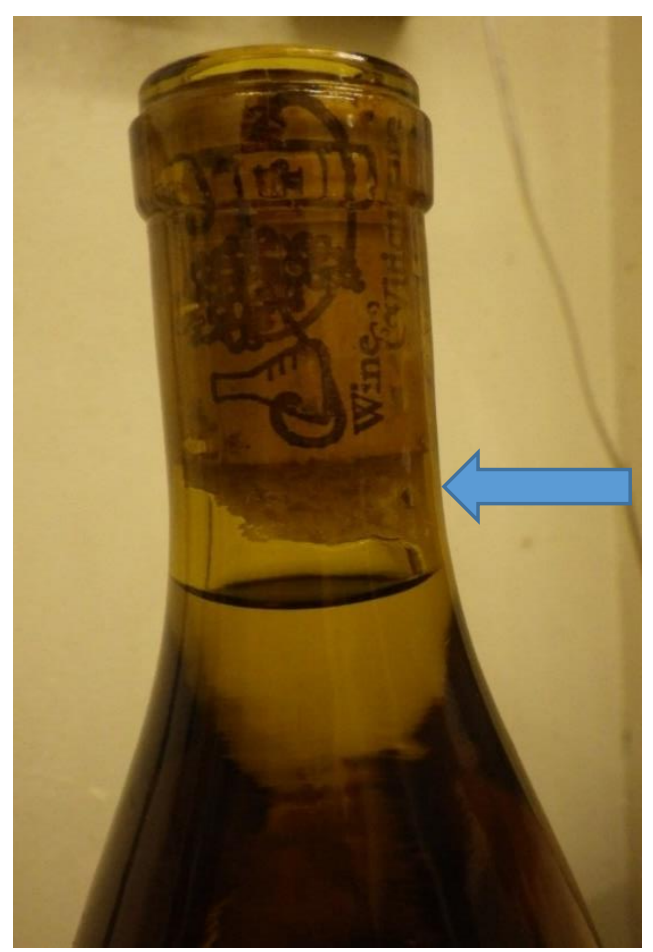

Figure 5. Tartrate crystal formation in wine due to instability.

2.3.2 Crystallization of KHT

The purpose of cold treatment is crystallization of KHT in unstable wine. Many factors affect crystallization as mentioned before, but its effects will be studied in more detail. One way to overcome the nucleation energetic barrier for crystallization to being is to provide sufficient agitation. By both mixing and seeding the wine with KHT crystals, a method referred to as cold stabilization contact process, the crystallization rate is accelerated (Rodriguez-Clemente et al. 1988). The amount of KHT crystals added for seeding is enough to overload the wine solution to make it supersaturated (Zoecklein et al. 1990). The optimal amount and size of the KHT crystals used for seeding was determined to be about $40 \mu \mathrm{m}$ at $4 \mathrm{~g} / \mathrm{L}$ (Garcia-Ruiz et al. 1991; Lasanta and Gómez 2012). According to Dunsford and Boulton (1981), the rate of crystallization happens following these kinetics $\frac{d m}{d t}=k_{d}(A)(C-C i)$. A is the surface area of the nuclei, $\mathrm{C}-\mathrm{Ci}$ is the degree of supersaturation, and $k_{d}$ is the mass transfer coefficient. The moment KHT 
crystals are added, the degree of supersaturation is so large that the crystallization rate depends only on the surface interface area and is at its most rapid rate. To have maximum area of contact, agitation and mixing of the solution to suspend the crystals homogeneously is needed. Once nuclei crystals have grown, the degree of supersaturation decreases and so does the rate of crystallization. By the end of the treatment process, the crystallization rate is controlled more by thermodynamics than kinetics (Ribereau-Gayon et al. 2006). According to the rate of crystallization kinetics, the degree of supersaturation, the particle size of KHT and concentration, agitation, temperature, and contact time are all important factors (Dunsford and Boulton 1981).

\subsubsection{Crystallization of CaT}

Not all of the above is necessarily true for CaT, which is considerably less soluble than KHT. It does not crystallize at the same rate as KHT and cannot be cold stabilized. CaT spontaneous nucleation takes much longer and usually occurs in wine after several years (Ribereau-Gayon et al. 2006). Other methods of stabilization such as electrodialysis and ion exchange must be used to ensure CaT crystal stability.

\subsubsection{Different Process Variations of Cold Stabilization}

Based on crystallization kinetics, variations of the cold stabilization technology have been developed. One way is the described above seeding of KHT in the standard batch stainless steel tanks. This reduces the amount of time it takes for wine to become stabilized from a week to only a few days, and allows for slightly higher temperatures to be maintained, therefore using less energy and time (Ribereau-Gayon et al. 2006). Seeding with KHT is fairly popular in the industry because of these benefits. 
Another innovation is rapid cold stabilization (Figure 6). This technology takes advantage of the contact process with KHT crystals. The process is the same as the standard cold treatment stabilization except wine flows through a column containing KHT crystals first then ends up in a holding tank for further precipitation. The purpose of this is to remove any impurities and inhibitors to increase the ease and speed of KHT crystallization (Rodriguez-Clemente et al. 1988). This rapid cold stabilization reduces the amount of processing time, wine loss, and energy consumption needed to stabilize the wine (Roget 2012). This process has only been done at the pilot scale and has not been widely adopted yet. The slow adoption for new cold stabilization technology is most likely due to the capital needed for new equipment and that the KHT in the crystal column must be cleaned and regenerated quite often (Rodriguez-Clemente et al. 1988). The KHT crystal surfaces in the absorption column must be cleaned after each run to maintain its effectiveness.

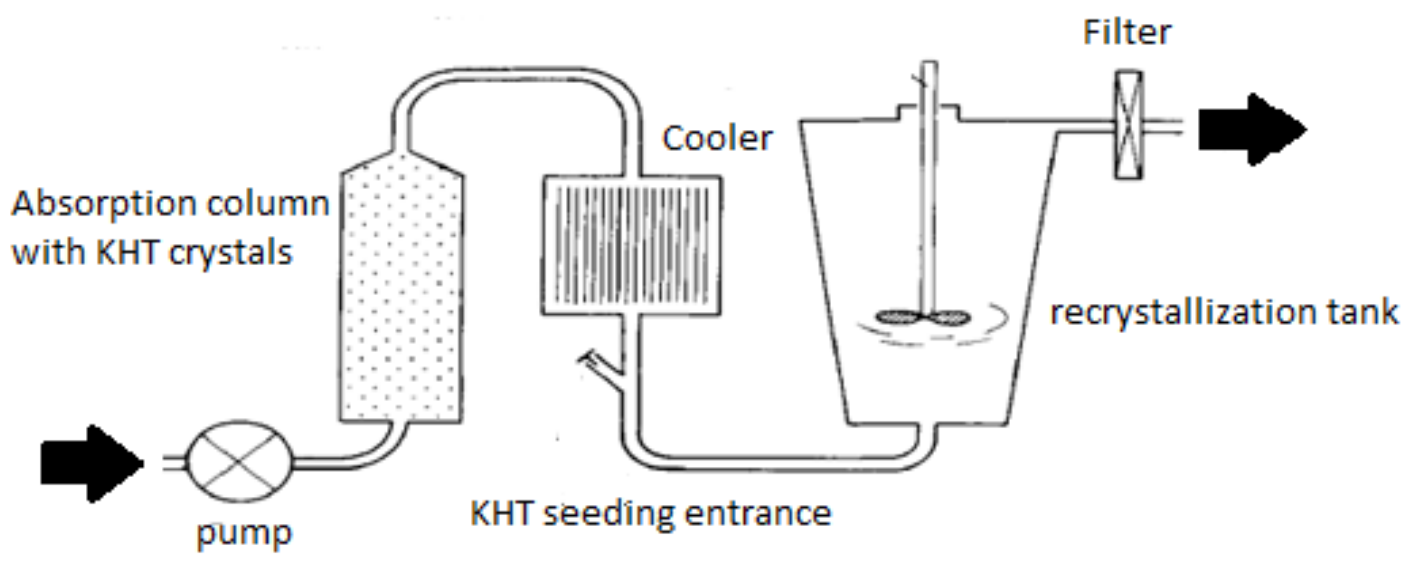

Figure 6. Continuous contact system stabilization process. Adapted from Rodriguez-Clemente et al. 1988. 
Cold stabilization of wine has been used for a long time and is somewhat regarded as tradition. Tradition in the wine industry is very important to consumers and producers, but more than that, varying the treatment of wine could affect its sensory evaluation and chemical composition. With this being the original method of tartrate stabilization, the wine produced by cold stabilization is accepted everywhere. For newer methods, like certain additives and ion exchange, acceptance by the International Organization of Vine and Wine (OIV) has been slow. Due to OIV controlling sales and protocol of wine internationally, cold stabilization is still the most common and accepted method. Not solely due to the OIV regulations, but sensory evaluations have proven that electrodialysis, ion exchange, and additive treated wines (Bosso et al. 2010) have different flavor (Lasanta et al. 2013), mouthfeel (Coulter 2013), aroma (Gómez Benítez et al. 2003), and color (Walker et al. 2004) compared to cold stabilized wine.

\subsubsection{Issues with Cold Stabilization}

While cold stabilization is widely accepted and most commonly used, there are many factors that make it seem outdated. The main issues being the process time, energy consumption, and waste removal. Even if seeding with KHT, the process time is still more than 48 hours, and the average time for standard cold stabilization is a week (Ribereau-Gayon et al. 2006). For every batch of wine, a tank with refrigeration is occupied for an extended period of time which results in a potential loss in production.

The other problem with the long process time is the amount of energy required for these thousand liter tanks of wine to be kept at around $0{ }^{\circ} \mathrm{C}$ for a week. The calculated total energy consumption for cold stabilization was found to be around $10 \mathrm{Wh}$ per $\mathrm{L}$ (Low et al. 2008), while electrodialysis was found to be between 1 and $2 \mathrm{Wh}$ per $\mathrm{L}$ 
(Bories et al. 2011). The importance of using less energy intensive processes is growing as energy costs rise due to the depletion of non- renewable energy sources.

Another issue cold stabilization creates is the use of water and caustics needed to remove KHT crystals on the walls of the holding tank and to clean the diatomaceous earth filter. About $3 \mathrm{~g}$ of caustics per L of liquid waste effluent is produced (Low et al. 2008), and 0.17 L of water per L of wine is used for cleaning purposes (Bories et al. 2011). The product and waste stream could also be centrifuged in order to collect the KHT crystal to either reuse them or sell as a byproduct. The caustics and diatomaceous earth in the waste stream must be treated and removed appropriately (Low et al. 2008).

\subsection{Alternative Stabilization Technology}

Cold treatment is not the only way to stabilize wine. Another way it is done is by using additives besides KHT, such as metatartaric acid, carboxymethyl cellulose, and yeast mannoproteins. However, some people frown upon additives because it is adding additional components to the wine that are not naturally occurring. Due to those opinions, the use of alternative stabilization technology such as ion exchange and electrodialysis are also viable options. These technologies remove the components that contribute to tartrate instability.

\subsubsection{Additives used for tartrate stability}

Metatartaric acid (MTA), carboxymethyl cellulose (CMC), and yeast mannoproteins are mixed with the wine for at least 48 hours at around $16{ }^{\circ} \mathrm{C}$ to fully dissolve and integrate with wine (Coulter 2013). If the wine filtered too soon, the additives will lose their colloid protective effects. All of these additives are used as crystallization inhibitors. Also, these additives do not negatively affect any of the sensory 
attributes (Lasanta et al. 2013). The main reason for studying the use of additives for tartrate stabilization is because of its reduced processing time, energy requirement, and price (Lasanta and Gómez 2012).

\subsubsection{Metatartaric acid (MTA)}

MTA (Figure 7) is a polyester derived from esterification of tartaric acid when heated (Ribereau-Gayon et al. 2006). It opposes the growth of KHT crystals by blocking the crystal building process. The problem with MTA is that it is fairly unstable and will slowly hydrolyze back to tartaric acid. This phenomenon is highly dependent on the temperature at which the wine is stored. Wine at $0{ }^{\circ} \mathrm{C}$ can stay stable for several years, where wines at $25^{\circ} \mathrm{C}$ only last about a month (Zoecklein et al. 1990). Another problem is that this additive is not permitted to be used in America, but is commonly used in Europe (Galpin 2006). A favorable characteristic about MTA is its ablility to prevent CaT instability.

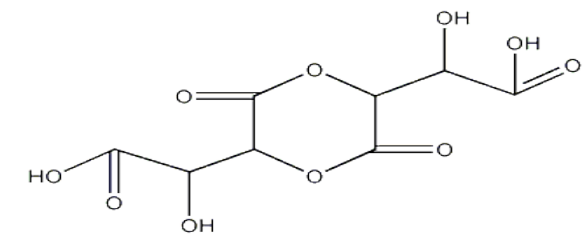

Figure 7. Metatartaric acid structure, not in polymer form.

\subsubsection{Carboxymethycellulose (CMC)}

In addition to the previous additives, $\mathrm{CMC}$ can be used as an additive that inhibits crystallization. CMC (Figure 8) is a polymer of cellulose rings substituted by carboxymethyl organic acid chemical groups often saturated by sodium (Claus et al. 2014). CMC reduces crystal growth rate significantly and is just as stable as metatartaric acid but is able to withstand higher temperatures and remain stable (Ribereau-Gayon et 
al. 2006). Another positive aspect is that $\mathrm{CMC}$ requires relatively low concentrations of about $2 \mathrm{mg} / \mathrm{L}$. However it can only be used for white wines, as there are complications in red wine because of the $\mathrm{CMC}$ reacting with the polyphenols and generating turbidity and a color change (Claus et al. 2014). Also, CMC is unable to prevent CaT crystallization due to its crystallized surface being different from KHT (Coulter 2013). The use of CMC for stabilization was recently approved by the OIV and FDA in 2014.

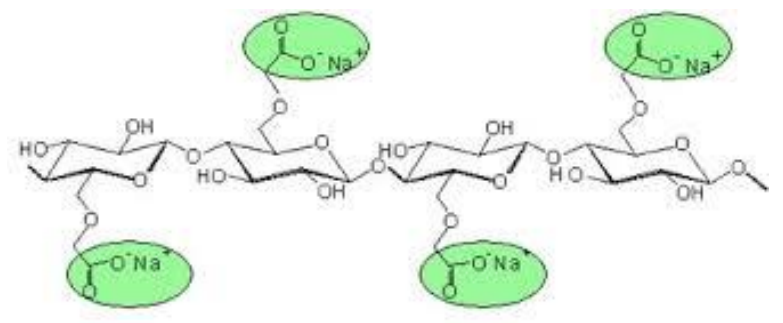

Figure 8. Carboxymethyl Cellulose chair structure.

\subsubsection{Mannoproteins}

Another additive that is commonly used are yeast mannoproteins. Mannoproteins are natural occurring glycoproteins with 15-90\% mannose found in the yeast, Saccharomyces cerevisiae, from the lees in the barrels used for aging wines. It acts as a protective colloid that inhibits tartrate crystallization (Lasanta and Gómez 2012). One difference between mannoproteins and metatartaric acid is that the mannoproteins are able to keep wines stable at higher storage temperatures unlike metatartaric acid (Ribereau-Gayon et al. 2006). However, mannoproteins are not very stable at temperatures below $0{ }^{\circ} \mathrm{C}$, where MTA is very stable. Another possible downside is that concentrations of more than $100 \mathrm{mg} / \mathrm{L}$ are needed to reach stabilization and the mannoproteins are unable to stabilize CaT crystallization. This additive is permitted for use by all countries for tartrate stabilization. 


\subsubsection{Ion Exchange Resin Process for Wine Stabilization}

Ion exchange technology is widely used today in many aspects of industry. It is mainly used for purification and extraction purposes. The basic nature of how ion exchange resin works is by exchanging ions from a mobile electrolyte and solid ion exchange material (Inamuddin and Luqman 2012). There are two types of ion exchangers, cation/acidic and anion/basic. Cation exchangers usually have sulfate functional groups and exchange positively charged ions with the electrolyte. Anion exchangers usually have tetrammonium functional groups and exchange negatively charged ions with the electrolyte (Inamuddin and Luqman 2012). The resin, the solid ion exchange material usually made of a polysterene, varies with bead and pore size depending on the mobile electrolyte solution. The ions exchanged from the electrolyte are held by the resin and are eluted after the process is done (Figure 9). The resin is then recharged to be basic or acidic accordingly.

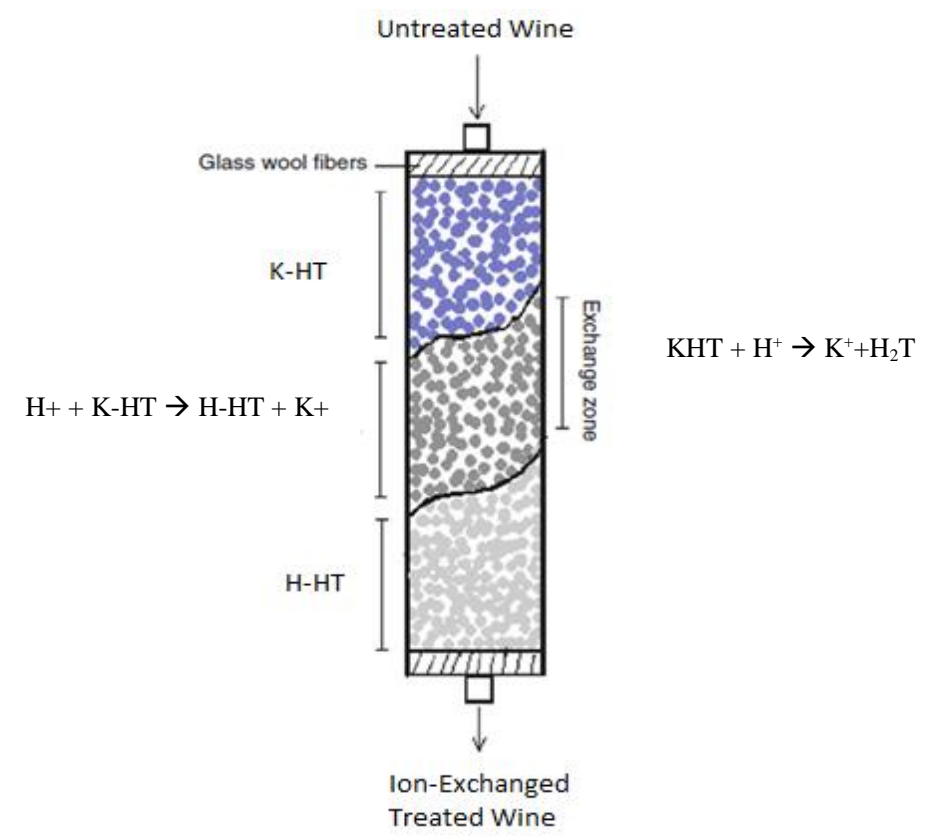

Figure 9. Diagram of a cationic exchanger used to stabilize wine by exchanging potassium for hydrogen ions. Adapted from Inamuddin and Luqman 2012. 
Cation exchangers are used to stabilize wine. Anion exchangers are not allowed by the OIV due to negative effects in the physiochemical composition and sensory evaluations (Mira et al. 2006; Lasanta et al. 2013). For cation exchangers, cations such as $\mathrm{Na}, \mathrm{K}, \mathrm{Mg}$, and $\mathrm{Ca}$ are exchanged for $\mathrm{H}+$ or $\mathrm{Na}+$, meaning tartaric acid concentration is not affected. Reducing potassium and calcium is the key to providing stability because without a supersaturated solution of KHT or CaT, crystal formation will not spontaneously occur (Benítez et al. 2002). A side effect that occurs due to H+ increase in wine is that the $\mathrm{pH}$ is often lowered by at most 0.3 after cation exchange, resulting in an increase in acidity (Walker et al. 2004). When the ion exchange resin is made with $\mathrm{Na}$, the sodium levels increase in the wine which can affect the sensory attributes of the wine. However, for cation exchange, no significant difference in sensory evaluations was determined when compared to cold treated wine (Mira et al. 2006). It is well known that $\mathrm{pH}$ values correspond to the color of wine, therefore ion exchange resins affect the color of wine which has lower hue and higher intensity values (Walker et al. 2004; Lasanta et al. 2013). The most important feature of cation exchange resins are that they provide great tartrate stability relatively quickly (Lasanta et al. 2013).

Even though this method seems to work well in terms of stability and sensory characteristics, cation exchange is still very new to the wine industry and was only recently permitted for use in 2012 by the OIV. Many are still cautious to use this technology because it will either acidify the wine or add sodium to the wine, which many believe affects the sensory characteristics of the wine (Walker et al. 2004; Lasanta et al. 2013). 


\subsubsection{Electrodialysis Process for Wine Stabilization}

Electrodialysis is a technology used to stabilize wine by using ion selective membranes and electrodes to create an electric potential to separate and extract cations and anions from the wine solution (Lasanta and Gómez 2012). Anionic and cationic membranes are alternatingly placed between the two electrodes with a spacing of between 300 and $700 \mu \mathrm{m}$. Wine and electrolyte solution, an aqueous sulfuric acid solution, in a parallel flow pass in ionic membrane separated channels at 1 Volt potential per cell (Figure 10).

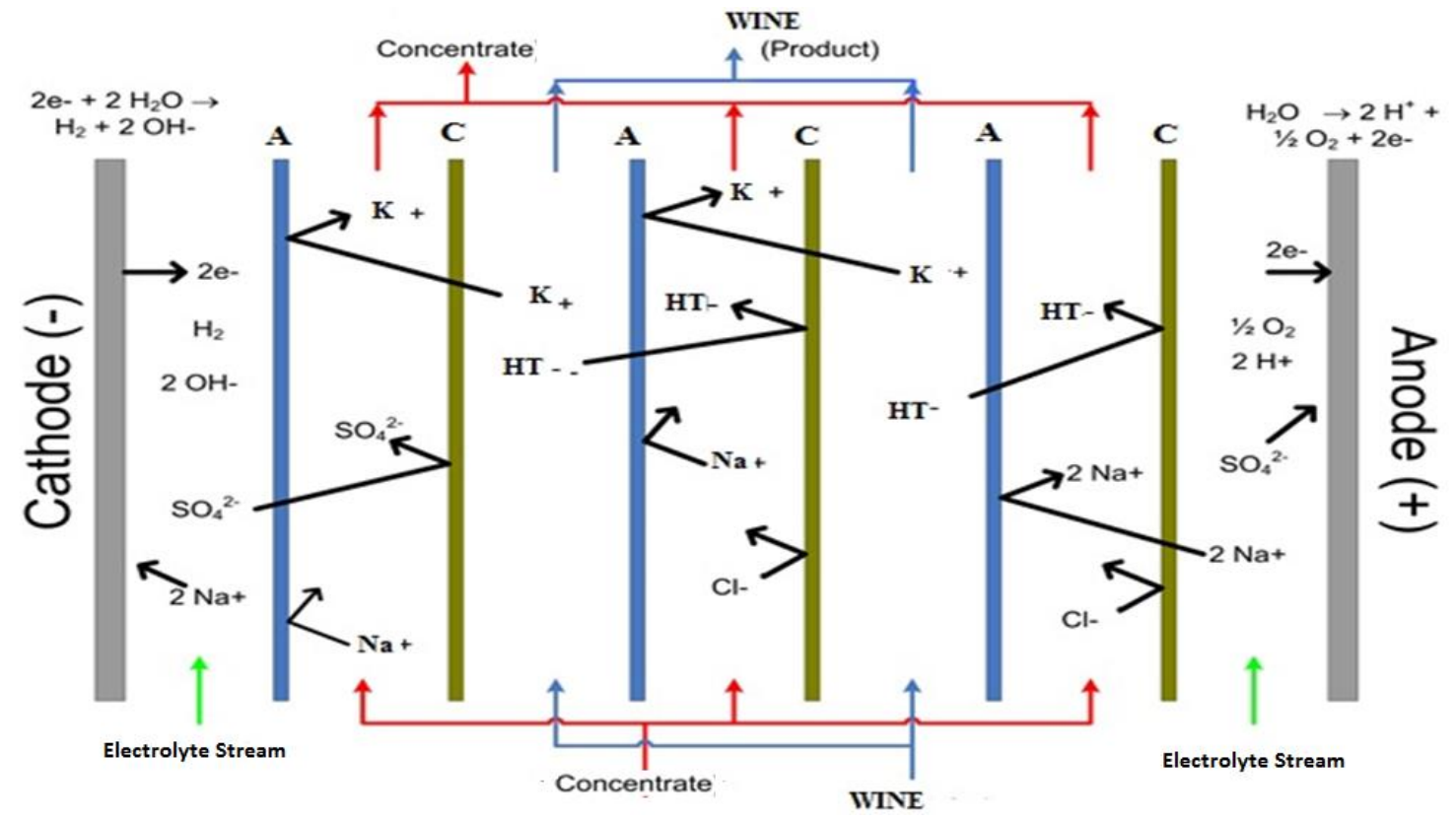

Figure 10. Electrodialysis system diagram for wine stabilization. $\mathrm{A}$ is the anionic membrane and $\mathrm{C}$ is the cationic membrane. Adapted from Lasanta and Gómez 2012

The anode attracts negative ions, like tartaric acid, while the cathode attracts positive ions, like potassium and calcium. However, cations cannot pass through anionic membranes, and anions cannot pass through cationic membranes. The ions extracted out of the wine pass through the designated membranes where they are concentrated into the electrolyte stream (Figure 10). 


\subsubsection{Energy, Water, and Waste Management}

Electrodialysis processing time and energy consumption needed to achieve tartrate stability is lower than cold stabilization (Low et al. 2008). According to one study, an electrodialysis system with reverse osmosis requires $0.05 \mathrm{~L}$ of water per L of wine and 2.1 Wh per L of wine (Bories et al. 2011). However, another study reported that electrodialysis required $0.2 \mathrm{~L}$ of water per $\mathrm{L}$ of wine and $8.0 \mathrm{Wh}$ per $\mathrm{L}$ of wine (Low et al. 2008). The majority of the water is used to transport the concentrated electrolyte (brine) in the system. The electrolyte system is made up of $\mathrm{NaCl}$ and sulfuric acid in order to provide a conductive medium and to lower the pH (Lasanta and Gómez 2012).

Estimated water usage was between 0.015 and $0.019 \mathrm{~L}$ of water/L of wine and the energy consumption was estimated to be between 10 and $17 \mathrm{Wh} / \mathrm{L}$ of wine for the cold treatment method (Bories et al., 2011). The water used in cold stabilization is for cleaning the tank and filters from KHT crystal deposits. The significantly larger energy consumption of cold stabilization relative to electrodialysis is due to bringing large volumes of wine to freezing temperatures for a prolonged period of time.

For both methods, a waste stream must be treated. In the case of electrodialysis, the brine stream contains concentrated acids and salts. In the case of cold stabilization, caustics are used to remove tartrate crystals from tanks. However for cold stabilization, the KHT crystals can be either reused or sold as cream of tartar with further processing, where elctrodialysis has no economical use for its byproducts (Low et al. 2008).

The water and energy resource usage for cold stabilization and electrodialysis vary from study to study. However, the general consensus is that cold stabilization 
requires a considerable amount more energy output but relatively less water than

electrodialysis (Table 1).

Table 1. Comparison of water and energy usage for cold stabilization and electrodialysis. (Pacific Gas and Electric Company 2007; Low et al. 2008; Bories et al. 2011)

\begin{tabular}{lll}
\hline Water and Electrical Usage & Cold Stabilization & Electrodialysis \\
\hline $\begin{array}{l}\text { Water (L of water per L of } \\
\text { wine produced) }\end{array}$ & $0.015-0.019$ & $0.05-0.2$ \\
$\begin{array}{l}\text { Electrical (Wh per L of wine } \\
\text { produced) }\end{array}$ & $10-17$ & $2.1-8.0$ \\
\hline
\end{tabular}

2.4.3.2 Deionization Degree and Stability

Electrodialysis is different from cold stabilization in the way it achieves stabilization. Electrodialysis mainly removes potassium, calcium, sulphates, and tartaric acid, while cold stabilization primarily removes potassium and tartaric acid. Also worth noting is that anions, malic, lactic, and acetic acid do not change significantly because their concentrations are so small compared to tartaric and sulphuric acids (Gonçalves et al. 2003). Even though electrodialysis removes more components from the wine, the reason why it can be seen as beneficial is because it removes calcium. Calcium is an important factor in the contribution to $\mathrm{CaT}_{2}$ crystals, which cause instability and form after a long time period compared to KHT. Cold stabilization does not remove calcium and therefore does not prevent this phenomenon. Another positive for electrodialysis is that it is able to control the amount of stability precisely, where cold stabilization cannot because crystallization rates are difficult to control. The way electrodialysis controls stability of the wine is by the deionization degree, which is defined by Soares (2009) as

$$
\text { Deionization Degree }(\%)=\frac{\text { Initial Conductivity-Final conductivity }}{\text { Initial conductivity }} * 100 \text {. }
$$

Wine treated at various deionization degrees resulted in different degrees of stabilization. A trend was found that the higher the deionization degree for three different types of 
wine, the more potassium, calcium, and tartaric acid was removed (Gonçalves et al. 2003). With less of these major components that contribute to crystallization and instability, it can be inferred that the wine is inherently more stable. Another way to show that the wine became more stable as the degree of deionization increased, was determined by its correlation with the saturation temperature (Soares et al. 2009). Therefore, electrodialysis can achieve a specific degree of stability and also ensure stability quickly, something cold stabilization cannot do. The type of wine affects process time and the degree of deionization needed to reach stability, but a rough estimate is that wines need to be at approximately $20 \%$ deionization degree (Gómez Benítez et al. 2003).

To test the effects of varying degrees of deionization on tartrate stability, four different wines were tested, white, rose, red, and fortified (Soares et al. 2009). The freezer test and saturation temperature were used to determine stability. Soares et al. (2009) concluded that different types of wines have different inherent stability properties, where white and rose are more unstable than red and fortified wines because they have less colloidal protection. The trend is clear that the higher the degree of deionization the more stable the wine is, because there were no precipitates in the freezer test and the saturation temperature followed an inverse relationship (Soares et al. 2009). For white wines with a deionization degree of $0 \%$, the saturation temperature was $19{ }^{\circ} \mathrm{C}$, and with a deionization degree of $30 \%$ it was $6.2{ }^{\circ} \mathrm{C}$. For Rose wines with a deionization degree of $0 \%$, the saturation temperature was $20{ }^{\circ} \mathrm{C}$, and with a deionization degree of $30 \%$ it was $2.7^{\circ} \mathrm{C}$. 


\subsubsection{Sensory Characteristics}

Flavor and aroma of wine are perhaps more critical for wine quality than stability. If a method results in unsatisfactory sensory evaluations, then the specific stabilization process is not viable. When wine is treated with electrodialysis, there were no significant differences in color, aroma, and flavor when compared with cold stabilized wine (Gonçalves et al. 2003). However, there have been contradicting studies that state there is a slight loss in aroma and flavor when treated with electrodialysis relative to cold stabilization, but still at an acceptable level (Gómez Benítez et al. 2003). Overall, the sensory evaluations for wine treated by electrodialysis are satisfactory and are backed up by the fact that the OIV has accepted it as a practice.

\subsection{Economics of Various Wine Stabilization Technologies}

The cost of each technology is the operating cost which includes energy, water, chemicals, labor, wine loss, and maintenance. However, each economic review does not cover all of the same components in its operating cost estimation. Standard cold stabilization cost of dollars per liter of wine produced is compared to electrodialysis, ion exchange, CMC, MTA, and Mannoproteins (Table 2).

Table 2. Cost of stabilizing a liter of wine for various stabilization technologies. (Agrovein 2012; Bories et al. 2011; Gomez Benitez et al. 2003; Lasanta and Gomez 2012; Low et al. 2008; Pacific Gas and Electric Company 2007)

\begin{tabular}{ll}
\hline Stabilization Technology & $\mathbf{\$} / \mathbf{L}$ of wine \\
\hline Cold Stabilization & $0.01-0.02$ \\
Electrodialysis & $0.01-0.05$ \\
Ion-Exchange & $0.001-0.003$ \\
CMC & $0.006-0.008$ \\
MTA & $0.0008-0.001$ \\
Mannoproteins & 0.03 \\
\hline
\end{tabular}

The economic benefits of electrodialysis are not agreed upon. On one hand, it is seen as less expensive because of its energy and time savings compared to cold 
stabilization (Pacific Gas and Electric Company 2007; Bories et al. 2011). One company's economic evaluation even reported that cold stabilization costs about 0.012 dollars per liter, while electrodialysis was about 0.0098 dollars per liter (Agrovin 2012). On the other hand, electrodialysis uses more water than cold stabilization, more energy than theoretically predicted, and the capital cost of the equipment is more than twice as expensive as the already established cold stabilization tanks (Low et al. 2008). Two of the more thorough economic reviews were done on various tartrate stabilization methods, and cold stabilization was determined to be the more economical than electrodialysis in both. The estimated cost of cold stabilization was between 0.01 and 0.02 dollars per liter and for electrodialysis it was between 0.012 and 0.05 dollars per liter (Gómez Benítez et al. 2003; Low et al. 2008).

Ion exchange is relatively new to the wine stabilization process but it has been verified to work and is very cost effective compared to cold stabilization. There are very low electricity and water costs. Most of the cost comes from chemical materials and waste management. The throughput of stabilized wine is also equivalent with other stabilization methods with at least $7000 \mathrm{~L}$ per hour. Ion exchange is at least 10 fold cheaper than cold stabilization with a cost of 0.001 to 0.003 dollars per liter compared to 0.01 to 0.02 dollars per liter (Agrovin 2012; Lasanta and Gómez 2012). Even though, ion exchange has such a large economic advantage, wineries are slow to adapt and cautious of anything that could potentially degrade the quality and sensory attributes of the wine. Some studies have shown there are not significant differences in sensory characteristics, however it is true that either the acidity will increase if using $\mathrm{H}+$ resins or the sodium concentration will increase if using Na resins (Mira et al. 2006). 
CMC has a relatively low cost of about between 0.006 to 0.008 dollars per liter of wine (Agrovin 2012; Lasanta and Gómez 2012). The reason for the economic advantage is that the cost is only the additive itself, and not any other operating costs. Also, the concentration of CMC needed to stabilize wine is relatively low. However, it can only be used for white wines. MTA is the cheapest process to stabilize wine at 0.0008 to 0.001 dollars per liter (Agrovin 2012; Lasanta and Gómez 2012). MTA is produced by heating and polymerizing KHT. Even though it is inexpensive, it does not produce stable wine at low temperatures and is also not allowed for use in the United States.

Mannoproteins cost per liter of wine produced was found to be more expensive than regular cold stabilization at around 0.03 dollars per liter (Lasanta and Gómez 2012). This is mainly due to the high concentrations needed to stabilize wine.

\subsection{Electrolysis Process}

The electrolysis process is very similar to electrodialysis. It makes use of two electrodes, one anode and one cathode, with a running electric current through an aqueous solution (Figure 11).

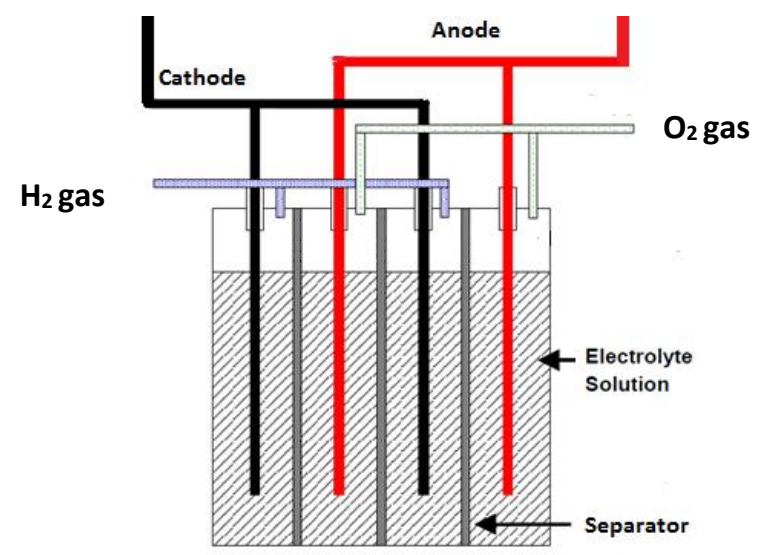

Figure 11. Conventional electrolyzer configuration of water. Adapted from Grimes 2008.

Electrolysis is commonly used to electrolyze water into hydrogen gas and oxygen by the following equation from Gimes (2008) $\mathrm{H}_{2} \mathrm{O}+$ electrical energy $\rightarrow \mathrm{H}_{2}(\mathrm{~g})+1 / 2 \mathrm{O}_{2}$ 
(g). Pure water is typically not used in electrolysis because it is a poor ionic conductor, so some electrolytes are usually added (Grimes et al. 2008). Hydrogen, which is a positive ion, is reduced at the cathode to form hydrogen gas. The hydrogen can then be used to create more energy. Water is oxidized at the anode to produce oxygen gas. There is also a separator between the anode and cathode that helps prevent mixing of evolved hydrogen and oxygen gases, but still allows the passage of electrolyte solution. The separator, usually a polymer, must allow the passing of liquid solution to have current and electrons flow to complete the circuit, but have small enough pores to not allow the evolved gas bubbles to pass.

Electrolysis is very similar to electrodialysis except for the reactants and products used. One difference is electrodialysis prevents the electrodes from coming into direct contact with any species other than the electrolyte with its ionic membrane. This helps it from developing any build up or scaling on the surface of the electrodes, which could potentially affect its performance and efficiency. In order to overcome this problem when using electrolysis, a method that repulses any attached species on the electrodes was used (Tanaka and Tatsuya 2008).

\subsubsection{Potential Application of Electrolysis for Wine Stabilization}

The method of removing mineral content from waste water by electrolysis has slowly become a more popular way to treat water. The minerals are usually cations like $\mathrm{K}^{+}$and $\mathrm{Ca}^{2+}$ that are attracted to the cathode at the same time as $\mathrm{H}^{+}$. The minerals attach to the electrode and form a scale on the surface, therefore leading to clean water. The deposition of the minerals on the electrode builds up on the surface and are removed quickly by reversing the polarity of the electrodes at $20 \mathrm{~V}$ and $0.5 \mathrm{~A}$ (Tanaka and Tatsuya 
2008). The positive cations that are attached to the surface will be repelled off the surface of the electrode if it is polarized positive. The scale precipitates thus fall off the electrodes with ease into water that is now mineral rich and discharged as waste (Figure 12 and 13).

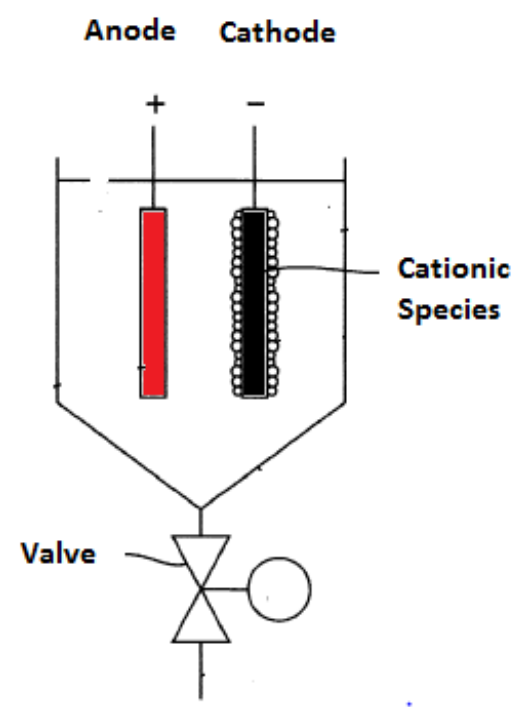

Figure 12. Attracting of cationic species with a cathode electrode. Adapted from Tanka and Tatsuya 2008

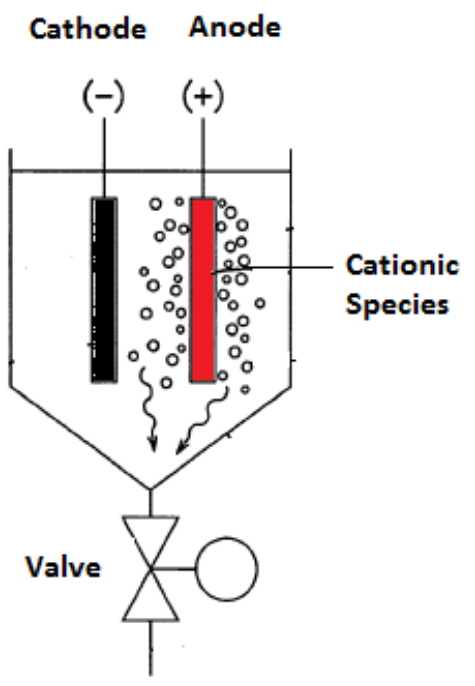

Figure 13. Repulsion of cationic species by changing the polarity of the electrodes. Adapted from Tanaka and Tatsuya 2008

This method of extracting minerals from wastewater can be applied to removing certain minerals from wine as well, because wine is made of mostly water (Tanaka and Tatsuya 2008). While these examples of electrolysis are done with water, there is already an application of electrolysis use with wine. Currently, it is only used to artificially control the aging process of wine. The aging process is replicated by having microoxidation occurring in the wine by applying a low current with electrolysis. The oxygen generation rate can be directly controlled by the amount of current passed, which is what causes the chemical changes in the aging process. At low currents of $6144 \mu \mathrm{A}$ and voltage of around $2 \mathrm{~V}$, species like polyphenols, ethanol, and sulfates are attracted to the surface of the electrodes (Fell et al. 2007). 
If run at higher currents, the same species attracted in electrodialysis are believed to be attracted to the electrodes for electrolysis, which would mainly be $\mathrm{K}, \mathrm{Ca}$, tartaric acid, and sulfates. Therefore, it can be inferred that using an electrolysis process on wine could potentially be used to stabilize wine. Possibly the process could be just as effective as electrodialysis but would use less water due to the electrolysis process not needing a continuous electrolyte and concentrate stream. 
CHAPTER 3

MATERIALS AND METHODS

\subsection{Materials}

Table 3. Summary of materials used.

\begin{tabular}{|c|c|c|c|}
\hline Materials & Source & Address & Purpose \\
\hline $\begin{array}{l}\text { Potassium } \\
\text { Bitartrate (KHT) }\end{array}$ & Spectrum & Gardena, CA & $\begin{array}{l}\text { To seed wine in } \\
\text { conductivity test. }\end{array}$ \\
\hline $0.1 \mathrm{M} \mathrm{NaOH}$ & FisherScience & Hanover Park, IL & $\begin{array}{l}\text { For Titration of } \\
\text { wine. }\end{array}$ \\
\hline $\begin{array}{l}\text { Nitric Acid } 70 \% \\
\text { Trace metals }\end{array}$ & FisherScience & Hanover Park, IL & $\begin{array}{l}\text { To make ICP } \\
\text { samples and clean } \\
\text { graphite. }\end{array}$ \\
\hline $\begin{array}{l}\mathrm{pH} \text { buffers }(3.00, \\
4.00,7.38,9.18)\end{array}$ & Ricca Chemicals & Arlington, TX & $\begin{array}{l}\text { To calibrate } \mathrm{pH} \\
\text { meter. }\end{array}$ \\
\hline $\begin{array}{l}\text { ICP Calibration } \\
\text { standard } 34 \\
\text { (K,Ca,Mg,Na } 5000 \\
\text { ppm, 5\% nitric acid } \\
\text { solution) }\end{array}$ & Inorganic Ventures & Christiansburg, VA & $\begin{array}{l}\text { To make ICP } \\
\text { standards. }\end{array}$ \\
\hline $\begin{array}{l}\text { Ethanol } \\
\text { (Histological } \\
\text { Grade) }\end{array}$ & FisherScience & Hanover Park, IL & $\begin{array}{l}\text { To make ICP } \\
\text { standards. }\end{array}$ \\
\hline $\begin{array}{l}\text { COD standard } \\
\text { range vials (20-900 } \\
\text { mg) }\end{array}$ & Bioscience, Inc. & Allentown, PA & $\begin{array}{l}\text { To measure organic } \\
\text { compounds in } \\
\text { solution. }\end{array}$ \\
\hline $\begin{array}{l}\text { Potassium Acid } \\
\text { Phthalate }\end{array}$ & Spectrum & Gardena, CA & $\begin{array}{l}\text { To make standard } \\
\text { solutions for COD } \\
\text { analysis. }\end{array}$ \\
\hline $\begin{array}{l}\text { Untreated } \\
\text { Chardonnay Wine } \\
\text { '14 }\end{array}$ & Cellar 360 & Paso Robles, CA & $\begin{array}{l}\text { To treat with } \\
\text { electrolysis }\end{array}$ \\
\hline $\begin{array}{l}\text { Cold Treated } \\
\text { Chardonnay Wine } \\
\text { '14 }\end{array}$ & Cellar 360 & Paso Robles, CA & $\begin{array}{l}\text { To compare with } \\
\text { electrolyzed wine }\end{array}$ \\
\hline $\begin{array}{l}\text { Deionized Water } \\
\text { (DI) }\end{array}$ & $\begin{array}{l}\text { Cal Poly San Luis } \\
\text { Obispo }\end{array}$ & $\begin{array}{l}\text { San Luis Obispo, } \\
\text { CA }\end{array}$ & $\begin{array}{l}\text { To wash equipment } \\
\text { and make } \\
\text { standards. }\end{array}$ \\
\hline
\end{tabular}


Table 4. Summary of equipment used.

\begin{tabular}{|c|c|c|c|}
\hline Equipment & Source & Address & Purpose \\
\hline Digital Multimeter & BK Precision & Yorba Linda, CA & $\begin{array}{l}\text { To record the } \\
\text { voltage and current } \\
\text { continuously. }\end{array}$ \\
\hline Cuvette & $\begin{array}{l}\text { BrandTech } \\
\text { Scientific, Inc. }\end{array}$ & Essex, CT & $\begin{array}{l}\text { To use for } \\
\text { colorimetry } \\
\text { readings. }\end{array}$ \\
\hline $\begin{array}{l}\text { Spectrophotometer } \\
\text { (Gensys 20) }\end{array}$ & ThermoScientific & Waltham, MA & $\begin{array}{l}\text { To measure the } \\
\text { absorbance for } \\
\text { colorimetry. }\end{array}$ \\
\hline $\begin{array}{l}\text { Graphite Electrodes } \\
\text { Grade: GM-10 }\end{array}$ & Graphite Store & Buffalo Grove, IL & $\begin{array}{l}\text { To attract ions to } \\
\text { its surface during } \\
\text { electrolysis }\end{array}$ \\
\hline $\begin{array}{l}\text { Low Voltage } \\
\text { AC/DC Power } \\
\text { Supply (SF-9584B) }\end{array}$ & PASCO & Roseville, CA & $\begin{array}{l}\text { To provide a set } \\
\text { voltage and current } \\
\text { to the electrodes }\end{array}$ \\
\hline $\begin{array}{l}\text { Peristaltic Pump } \\
\text { Model 77200-62 }\end{array}$ & Cole-Parmer & Chicago, IL & $\begin{array}{l}\text { To pump wine } \\
\text { continuously at a } \\
\text { set flow rate }\end{array}$ \\
\hline $\begin{array}{l}\text { pH/Conductivity } \\
\text { Meter } \\
\text { Model: } \\
\text { OrionstarA215 }\end{array}$ & ThermoScientific & Waltham, MA & $\begin{array}{l}\text { To record the } \mathrm{pH} \\
\text { and conductivity of } \\
\text { wine. }\end{array}$ \\
\hline $5 \mathrm{~mL}$ Pipette & ThermoScientific & Waltham, MA & $\begin{array}{l}\text { To accurately } \\
\text { measure volumes. }\end{array}$ \\
\hline COD reactor & Bioscience, Inc. & Allentown, PA & $\begin{array}{l}\text { To measure organic } \\
\text { compounds in } \\
\text { solution. }\end{array}$ \\
\hline $\begin{array}{l}\text { Adjustable Power } \\
\text { Resistor } \\
\text { Model: AVT100-50 }\end{array}$ & $\begin{array}{l}\text { Vishay Huntington } \\
\text { Electric Inc. }\end{array}$ & Shelton, CT & To control current. \\
\hline $\begin{array}{l}\text { Inductively Coupled } \\
\text { Plasma (ICP) } \\
\text { Model: Ultima } 2\end{array}$ & HORIBA & New Jersey, NJ & $\begin{array}{l}\text { To determine metal } \\
\text { ion concentrations } \\
\text { in wine. }\end{array}$ \\
\hline
\end{tabular}




\subsection{Methods}

\subsubsection{Electrolytic Process}

Untreated white wine was treated for tartrate stabilization under electrolysis at room temperature. The electrolysis system was set up with a graphite anode and cathode that were $2 \mathrm{~cm}$ apart, which were connected to the power supply, digital multimeter, and variable resistor (Figure 14). The reaction flask was then filled with $500 \mathrm{ml}$ of untreated white wine. A peristaltic pump was used to pump the wine from the top, where it was submerged in the wine, through the bottom up at a flow rate of $0.25 \mathrm{~L} / \mathrm{min}$ to be well mixed. A mercury in glass thermometer was placed on the side of the reaction flask to record the temperature throughout the experiment. Voltage and current were monitored and recorded by a digital multimeter. Voltage was kept constant at 24.5 volts and the current was controlled and varied to $0.2,0.3,0.4$, and 0.5 Amps by the power supply and variable resistor. The process time was varied at 1,2 and 3 hours (Table 5).

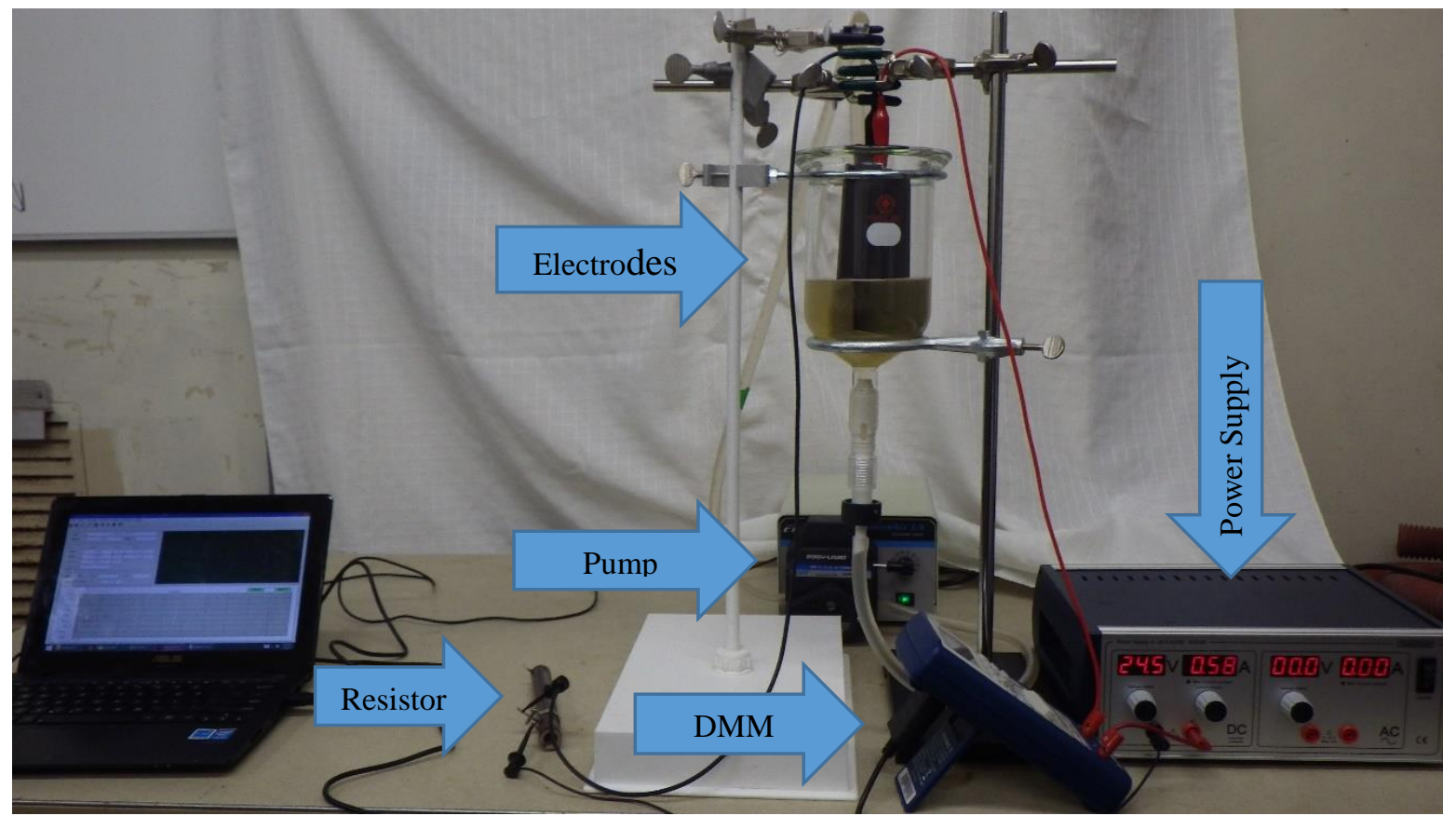

Figure 14. Experimental set-up for electrolysis treatment of wine. 


\subsubsection{Preliminary Experimental Process}

Preliminary experiments were carried out on 1 liter of Cal Poly 2010 Chardonnay Wine to determine a range for the voltage, current, and time needed to see any significant changes in the stability and composition of the wine when treated with an electrolytic process. The first test was run at 2.5 Volts and 0.01 Amps for 4 hours, because the assumption was that $\mathrm{H}_{2} \mathrm{O}$ would out compete the desirable cations at higher voltages and current. Another test was done at maximum possible voltage and current setting to test for any significant changes at 24.5 volts and 2.6 Amps. Afterwards, subsequent tests $(1.0 \mathrm{~A}, 2.5 \mathrm{hrs} ; 0.5 \mathrm{~A}, 1$ and $4.5 \mathrm{hrs} ; 3.6 \mathrm{~A}, 10 \mathrm{mins})$ were run at $24.5 \mathrm{~V}$ to determine a range for time and current for the experimental process to achieve both wine stability and quality (Table 5).

The constants in our study were the distance between the electrodes, the size and composition of the electrodes, and the flow rate of the wine. The reason these variables were kept constant was because the belief was that the voltage and current were the most important factors in achieving stability. Also, the trend for these constants was fairly well known, the closer the electrodes the less distance the particles need to travel and thus increasing the charge flow rate (Georgiev 2007). The larger the surface area of the electrode, the more area particles can interact with the electrode (Das et al. 2014). Preliminary experiments were also carried out on the washing sequence of the electrodes. After reversing the polarities of the electrodes and placing them into DI water, the voltage and current was set to the maximum parameters and ran for 15 minutes. According to Tanaka and Tatsuya (2008), the scaling on the electrodes were meant to be repulsed off the surface within 5 minutes, however this was not the case in our study. 
Therefore, the reverse polarity sequence was run until the scaling was detached from the surface and that was determined to be at most 60 minutes, depending on the amount of scaling. When all precipitates were visibly off the electrode, it was thought to be clean and not retain any particles.

Table 5. Experimental parameters: treatment number, time, current, and charge values.

\begin{tabular}{llll}
\hline $\begin{array}{l}\text { Treatment } \\
\text { Number }\end{array}$ & Time (hr) & $\begin{array}{l}\text { Current } \\
\text { (Amps) }\end{array}$ & $\begin{array}{l}\text { Charge } \\
\text { (Coulombs) }\end{array}$ \\
\hline 1 & 1 & 0.2 & 0.2 \\
2 & 1 & 0.3 & 0.3 \\
3 & 1 & 0.4 & 0.4 \\
4 & 1 & 0.5 & 0.5 \\
5 & 2 & 0.2 & 0.4 \\
6 & 2 & 0.3 & 0.6 \\
7 & 2 & 0.4 & 0.8 \\
8 & 2 & 0.5 & 1.0 \\
9 & 3 & 0.2 & 0.6 \\
10 & 3 & 0.3 & 0.9 \\
11 & 3 & 0.4 & 1.2 \\
12 & 3 & 0.5 & 1.5 \\
13 (Cold & & & \\
Treated) & & & \\
\hline
\end{tabular}

\subsubsection{Untreated and Treated Wine Analysis}

Wine Sample-All untreated wine samples were obtained from Cellar 360 on December 2014. The 36 wine samples were all Chardonnay wines but from 3 different tanks.

Therefore, the treatments were blocked for each tank to reduce known variability in the initial compositions of the different tanks or batches. This lead to 12 samples per tank or batch. A pair of cold stabilized wines were taken from each of the same tanks, however, the compositional make up of each tank was likely different than the wine taken initially because of the common practice to top off and mix wines in the tanks. 
Temperature-A mercury thermometer was placed in the reaction vessel and the temperature was recorded every 5 minutes from the start of every experiment until the end.

Tartaric Acid Concentration-A $50 \mathrm{~mL}$ sample of untreated wine, the electrolysis treated wine, and the cold treated wine were sent to ETSLABS for tartaric acid analysis follow their method. Liquid chromatography and mass spectrometry were used. Liquid chromatography ran under the conditions of $10 \mu \mathrm{L}$ injection volume, column specifications of Allure Organic Acids ( $250 \times 4.6 \mathrm{~mm}) 5 \mu \mathrm{m}, 60 \AA$, mobile phase of $0.5 \%$ Formic acid in water, flow rate of $0.7 \mathrm{~mL} / \mathrm{min}$, temperature of $50^{\circ} \mathrm{C}$, and isocratic mode. The internal standard was Tartaric-2,3-d2 Acid. The mass spectrometry ran under the source type of electrospray ionization with negative polarity. Samples and standards were all diluted 1:50 with DI water and internal standard was added. A linear calibration curve was established corresponding to 0.5 to $10 \mathrm{~g} / \mathrm{L}$ equiv. in sample.

Titratable Acidity-Following the Chemical analysis of grapes and wine: techniques and concepts (Illand 2004), a $10 \mathrm{~mL}$ sample of untreated wine, electrolysis treated wine, and cold treated wine each were placed into separate flasks to be degassed. The solutions were heated to the boiling point and then abruptly removed from heating and cooled to room temperature. The $\mathrm{pH}$ meter was calibrated before operating. In the $10 \mathrm{~mL}$ of degassed wine sample, additional DI water was added to the beaker to cover the probe. The $0.1 M \mathrm{NaOH}$ solution was titrated into the beakers with the degassed wine until the $\mathrm{pH}$ was about 8.2 while being constantly mixed. The volume of titrate was recorded and used to calculated the titratable acidity. 
pH-A $20 \mathrm{~mL}$ sample of untreated wine and electro-treated wine were placed into separate beakers and mixed with a magnetic stir bar for each treatment combination. The cold treated wine sample from the same batch was also analyzed. The $\mathrm{pH}$ meter was calibrated in 3.0, 4.0, 7.38, and 9.18 buffer solution before operating. The $\mathrm{pH}$ measurements were taken and recorded once the value stabilized.

Color Analysis-Following the color analysis method for wine (OIV 2009) a $3 \mathrm{~mL}$ sample of wine was used to fill a $10 \mathrm{~mm}$ quartz cuvette. The spectrophotometer was adjusted to $0 \%$ absorbance with DI water at 420,520, and $620 \mathrm{~nm}$ wavelengths. The samples were then placed in the spectrophotometer and absorbance readings at 420, 520, and $620 \mathrm{~nm}$ wavelengths were determined. At $420 \mathrm{~nm}$, the yellow spectrum is absorbed. At $520 \mathrm{~nm}$, the red spectrum is absorbed. This color analysis was done for the untreated wine, electrolysis treated wine, and cold treated wine. Hue, a description of the shade of the color, was measured as $\frac{A_{420}}{A_{520}}$. Intensity, the amount of saturation of the color, was measured as $\mathrm{A}_{420}+\mathrm{A}_{520}+\mathrm{A}_{620}$.

Ion-Coupled Plasma for Determining Mineral Composition -Analysis of mineral elements, potassium, calcium, magnesium, and sodium in wine samples followed the ICP-AES method (OIV 2013). ICP was performed to determine the potassium, sodium, magnesium, and calcium concentrations in the untreated wine, the electrolysis treated wine and the cold treated wine. The wine samples were prepared by making a 1:5 dilution with $1 \%$ nitric acid solution. The standards were created by using the ICP Calibration standard solution which contains a mixture of all four metal ions, $\mathrm{K}, \mathrm{Ca}, \mathrm{Mg}$, and $\mathrm{Na}$. First, the $100 \mathrm{~mL}$ volumetric flasks were soaked in $10 \%$ nitric acid solution for at least 12 hours, then dilutions were made accordingly to create $1,2,5,10,20,50,100,200$ ppm 
and blank standard solutions made of $2.5 \%$ ethanol and $1 \%$ nitric acid. A calibration step was done every time for the ICP prior to any analysis using the standards created. All samples were measured in triplicates. ICP was run at $1.3 \mathrm{~kW}$, plasma gas flow at $15 \mathrm{~L} / \mathrm{min}$, auxiliary gas flow of $1.5 \mathrm{~L} / \mathrm{min}$, nebulizer pressure: $200 \mathrm{kPa}$, stabilization period of 20 seconds, measurement time per replicate of 5 seconds, pump speed of $15 \mathrm{rpm}$, and rinsing time of 30 seconds.

Conductivity Test for Tartrate Stability Analysis-Following the Zoecklein et al. (1990) method, $80 \mathrm{~mL}$ samples of wine were maintained at $\approx 0{ }^{\circ} \mathrm{C}$ throughout the test by using an ice water bath. The initial conductivity of both treated, untreated, and cold treated wine were measured using the conductivity meter at $\approx 0{ }^{\circ} \mathrm{C}$ while stirring the solution with a magnetic stir bar. After the initial conductivity was measured, $1 \mathrm{~g}$ of KHT powder was added into the solutions while mixing. The conductivity was recorded every five minutes with the conductivity meter until the value stabilized or at a maximum of 35 minutes, to yield the final conductivity value. The difference between the final conductivity and the initial conductivity needed to be within $5 \%$ to be considered stable.

\subsubsection{Analysis of Wash Water}

Water Wash-After removing the treated wine, the electrodes were placed back into the reaction flask, but instead filled with $600 \mathrm{ml}$ of deionized water. The polarities of the graphite electrodes were reversed and the process was primarily based on Tanaka and Tatsuya (2008). This was run at 24.5 Volts and at the maximum current for 60 minutes at $2 \mathrm{~cm}$ apart. The electrolysis system was then shut off and removed from the beaker for the water sample to be collected for further analysis. The water samples were mixtures of precipitates so nitric acid was added to create a $1 \%$ nitric acid in water solution in order 
to obtain a homogeneous sample. The graphite electrodes were inspected for residual contaminates on the surface. Then the graphite electrodes were immersed and mixed in $500 \mathrm{~mL}$ of $2 \%$ nitric acid solution for 15 minutes to potentially remove any remaining precipitates. Water and acid samples were then analyzed by ICP and Chemical Oxygen Demand (COD).

Chemical Oxygen Demand (COD) -Following the Chemical Oxygen Demand Methods (Bioscience 2008) standards of 100, 250, 500 ppm were made with Potassium Acid Phthalate and DI water. Each standard had $2.5 \mathrm{~mL}$ pipette into COD vials. Then $2.5 \mathrm{~mL}$ of each sample of wash water were pipette into COD vials. The vials were then shaken and placed into a heating block at $150{ }^{\circ} \mathrm{C}$ for 2 hours. After the heat treatment, the vials were all cooled to room temperature by placing them in a water bath. Samples and standards were transferred into cuvettes to be analyzed by a spectrophotometer at $600 \mathrm{~nm}$ wavelength and their absorbance readings were determined. A calibration curve was created with the standards, and the sample concentrations were then calculated using the calibration curve.

\subsubsection{Statistical Method}

A total of 36 samples were used for the one factor 10 level experiment. The design of the experiment was a Randomized Complete Block Design with 3 blocks. ANOVA with Tukey comparisons was used to find significant differences between the charge treatments for all measured variables following the estimation method, restricted maximum likelihood (REML) and an overall significance level of $90 \%$ and individually at $99 \%$. A regression analysis was also done to determine linear correlations between 
charges with 10 levels and all measured variables. All analyses were performed with JMP (JMP, Pro 11, SAS, Cary, NC).

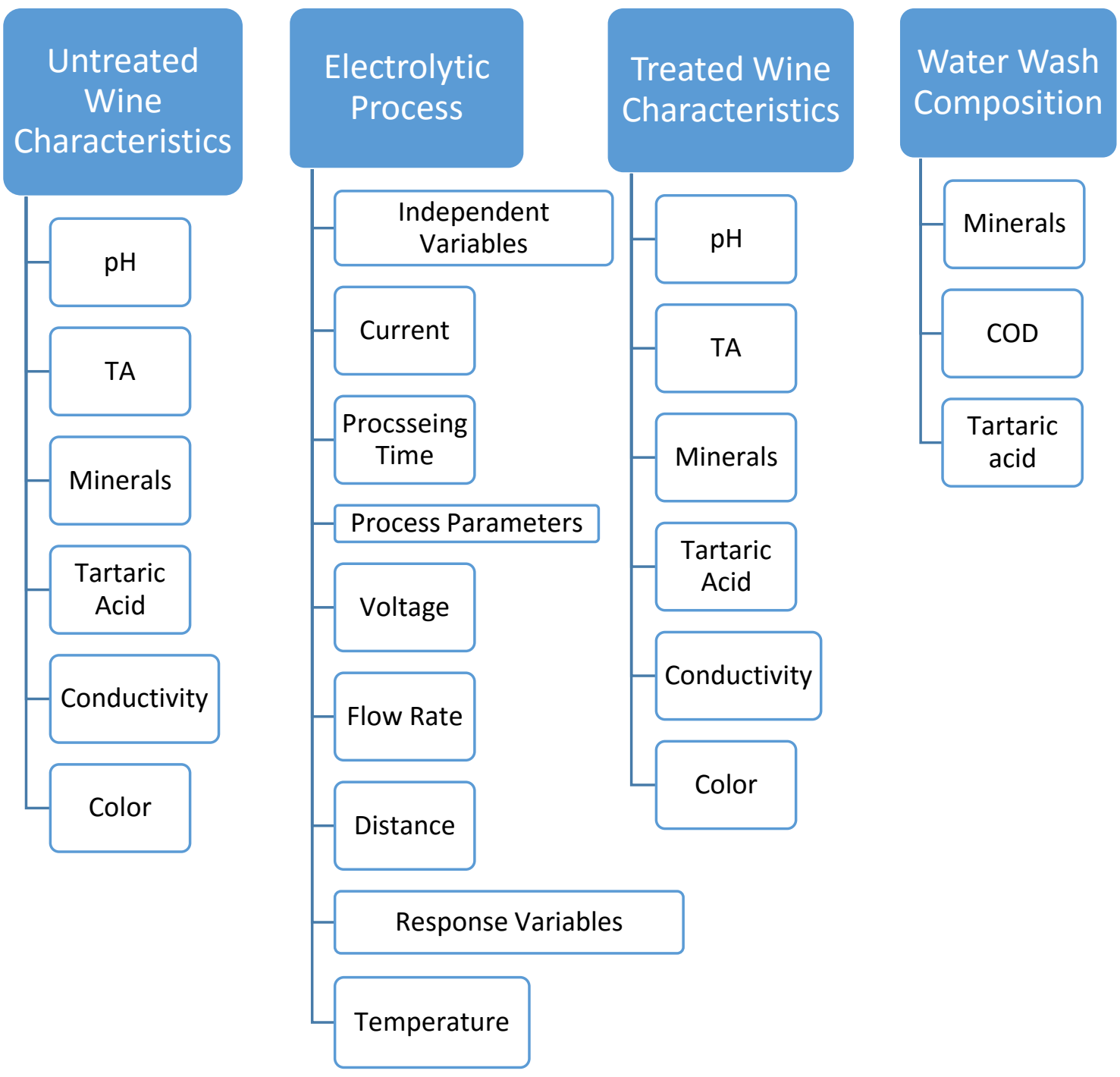

Figure 15. Flowchart of the overall experimental process and variables analyzed. 


\section{CHAPTER 4}

\section{RESULTS AND DISCUSSION}

\subsection{Determination of Analysis for the Electrolytic Process}

The preliminary test showed that with voltages of less than $4.0 \mathrm{~V}$ and currents of less than 0.1 Amps for 4 hours no change was observed in the wine's stability, acidity, or the visible surface of the electrodes. At the maximum output, 24.5 Volts and 2.6 Amps for 3 hours, there was a decrease in acidity, potassium and calcium ions, and visible scaling on the electrodes. The electrolytic process at the maximum output was found to be able to stabilize wine through the conductivity test. However, there was an apparent decrease in the quality of the wine based on basic aroma and color observations. These issues were most likely due to the increase in temperature of the wine from room temperature to $67^{\circ} \mathrm{C}$ (Dharmadhikari 2015).

Subsequent experiments were run in order to strike a balance between stability and quality and provided a rough estimate of the voltage, current, and time to run the process (Table 5). Time was set to have three levels, 1, 2, and 3 hours because 1 hour seemed to be the minimum time it took to see any changes and anything past 3 hours was seen as not a viable process. The current was determined to range between 0.5 to $1 \mathrm{Amp}$ per liter of wine. A value of 0.5 Amps was the minimum value where a change occurred in the stability. A value of 1 Amp was the maximum where adverse effects to the wine were first noticed. The power supply limited the options for voltage and current because they are in a direct relationship with each other so that the voltage or current could not vary without the other variable changing as well. At this point, the current looked to be a more important factor and could be varied with a power variable resistor without affecting the voltage, which was kept as a constant at 24.5 Volts. A range of processing 
times and currents were chosen to balance the effectiveness of stabilizing the wine and maintaining the integrity of the quality in the wine (Table 5). However, all analysis was done in terms of electrical charge (coulombs) after determining that different current time combinations with the same amount of charge did not result in any significant differences (Appendices).

In the preliminary experiments, the rise in temperature rate was significant and observed for increasing currents. Even though ampere seconds is equal to coulombs, in this study, processing time and current combination was important to distinguish. Some treatments with the same charges at different processing times and currents led to different final temperatures of the wine. The rate of charge passed, the current, is known to create heat when passed through resistance (Grimes et al. 2008). The increase in temperature affected the color and was known to affect the quality as well (Dharmadhikari 2015). The current and time combination treatments experimental design was used instead of charge because there was a belief that even with the same amount of charge transferred throughout the process, the rate in which it did so could lead to significantly different results. For example, treatment 3 and 5 were both 0.4 coulombs, treatments 6 and 9 were both 0.6 coulombs but different current and times (Table 5). After Tukey analysis of all the response variables for the treatments, it was determined that treatments with the same charge were not significantly different from each other (Appendices). Therefore, the final analysis was done in relation to charge. Also, initially the experiment was carried out to compare all electrolytic treatments to the cold treated wines. However, after analyzing the results it was determined that the cold treated wines 
initial composition was significantly different our study's initial wine so results could not be compared accurately (Appendices).

When running test wash sequences, sequential runs in DI water with the visibly clean plate resulted in some potassium ions leaching out from the electrode into the clean DI water. It was noticed that even with the water wash sequence and an additional nitric acid wash, it was difficult to prevent all leaching of all potassium ions from the electrodes. Though the electrodes were found to retain some of the minerals and possibly other components, it did not significantly affect the electrolytic process in its abilities to stabilize the wine. The cleaning process has the ability to not use any caustics if desired.

\subsection{Effect of Electrolytic Process on Temperature Rise of Wine}

The effect of electrical charge on the temperature of wine during the electrolytic process is shown in Figure 16. Tukey comparisons were done to identify which charge treatments were significantly different from each other in the mean differences of temperature after treatment (Table 6). The $\Delta$ Temperature is the temperature difference between the final electrolytic treated wine and the initial untreated wine for each sample. It appears that as charge increases, the temperature of the wine after electrolytic treatment increases. The trend appears to be increasing linearly (Figure 17). All treatments had an increase in temperature when going through the electrolytic process and were significantly different from the initial temperature of the wine with 99\% confidence (Figure 18). Any values outside the $99 \%$ confidence interval are considered significantly different from the specific charge treatment. All treatments led to an increase in temperature due to the treatment's confidence intervals being significantly different from initial temperature. The cause for the temperature increase was due to the heat created from the current running through the wine, which has a natural resistance (Grimes et al. 
2008). The system was open to the atmosphere and the temperature was not controlled.

Increased temperatures can lead to rapid oxidative browning which often times can alter the flavor and aroma (Dharmadhikari 2015). A cooling jacket could have been used to prevent increases in temperature, however, it was not used in our study because the desire was to not use more energy than necessary.

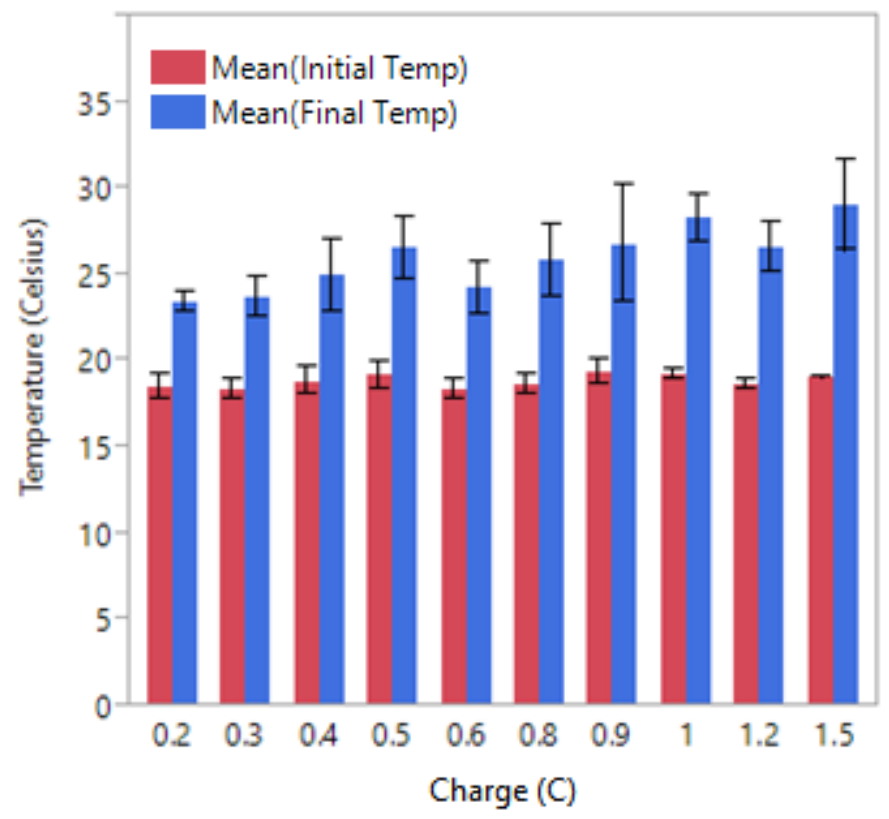

Figure 16. Comparison of the effect of electrical charge (coulombs) on the intial and final temperatures for before and after electrolytic treatment at one standard deviation.

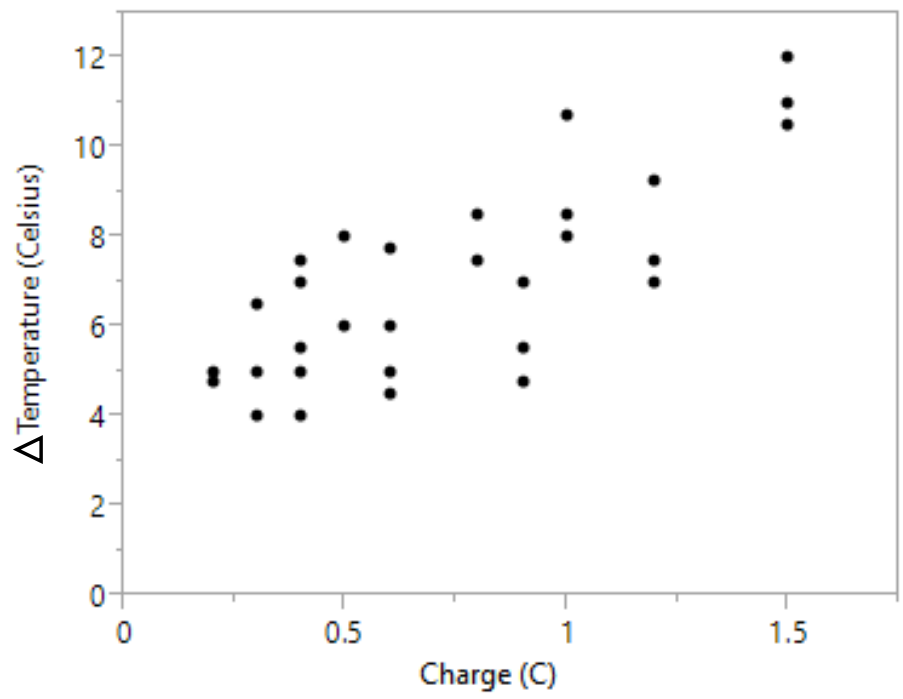

Figure 17. The effect of electrical charge (coulombs) on the temperature differences between the electrolytically treated and initial wines. 


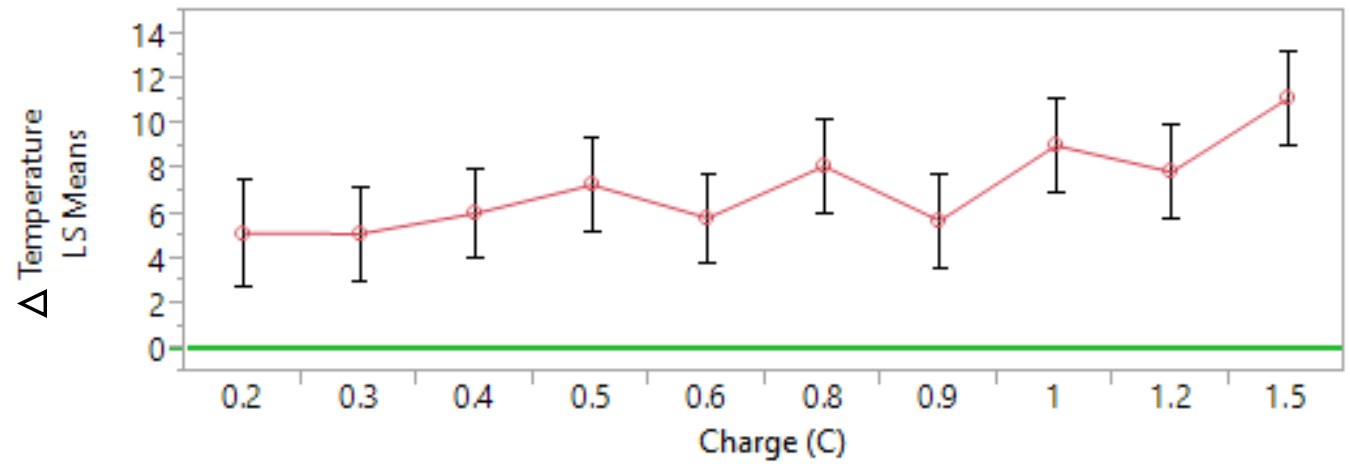

Figure 18. $\Delta$ Temperature least square means for different charges with $99 \%$ confidence intervals.

Table 6. Tukey comparison of charge treatments for mean differences in temperature.

\begin{tabular}{llllr}
\hline Charge & & & & $\Delta$ Least Sq Mean \\
\hline 1.5 & A & & & 11.166667 \\
1 & A & B & & 9.083333 \\
0.8 & A & B & C & 8.166667 \\
1.2 & A & B & C & 7.916667 \\
0.5 & & B & C & 7.333333 \\
0.4 & & B & C & 6.083333 \\
0.6 & & & C & 5.875000 \\
0.9 & & B & C & 5.750000 \\
0.2 & & B & C & 5.198058 \\
0.3 & & & C & 5.166667 \\
\hline
\end{tabular}

\subsection{Effect of Electrolytic Process on Tartaric Acid Reduction in Wine}

The effects of increasing charge on tartaric acid concentration is shown in Figure 19. Unlike $\Delta$ Temperature, the $\Delta$ Tartaric acid is the tartaric acid difference between initial untreated wine and the final electrolytic treated for each sample, which equals to the decrease of tartaric acid concentration. There is a statistically significant $(\mathrm{p}<0.0001)$ positive correlation between charge (Figure 20) and the amount of tartaric acid removed from the wine. As charge increases so does the amount of tartaric acid removed. The $\mathrm{R}^{2}$ value of 0.9 reinforces the fact that the linear correlation is strong and a good fit. In Figure 21, the mean tartaric acid concentration differences for each treatment with 99\% confidence intervals were compared with the baseline initial, where the tartaric acid difference between untreated wines was 0 . All treatments except $0.2,0.3$, and 0.4 coulombs were significantly different from the initial wine with $99 \%$ confidence (Figure 
21). The Tukey comparisons for all treatments were done in Table 7 to show any significant differences between any charge treatments for mean differences in tartaric acid. The importance of reduction in tartaric acid concentrations is because tartaric acid is directly related to all crystallization instabilities with the formation of KHT. The tartaric acid was removed via the anode of the electrolytic system, due to its electrostatic properties (Lasanta and Gómez 2012). When analyzing the electrodes wash water, tartaric acid was not found in a high enough concentration to be detectable for any treatment. Therefore, tartaric acid was most likely oxidized by losing two hydrogens and four electrons at the anode and becoming dioxosuccinic acid according to previous literature (Song et al. 2012).

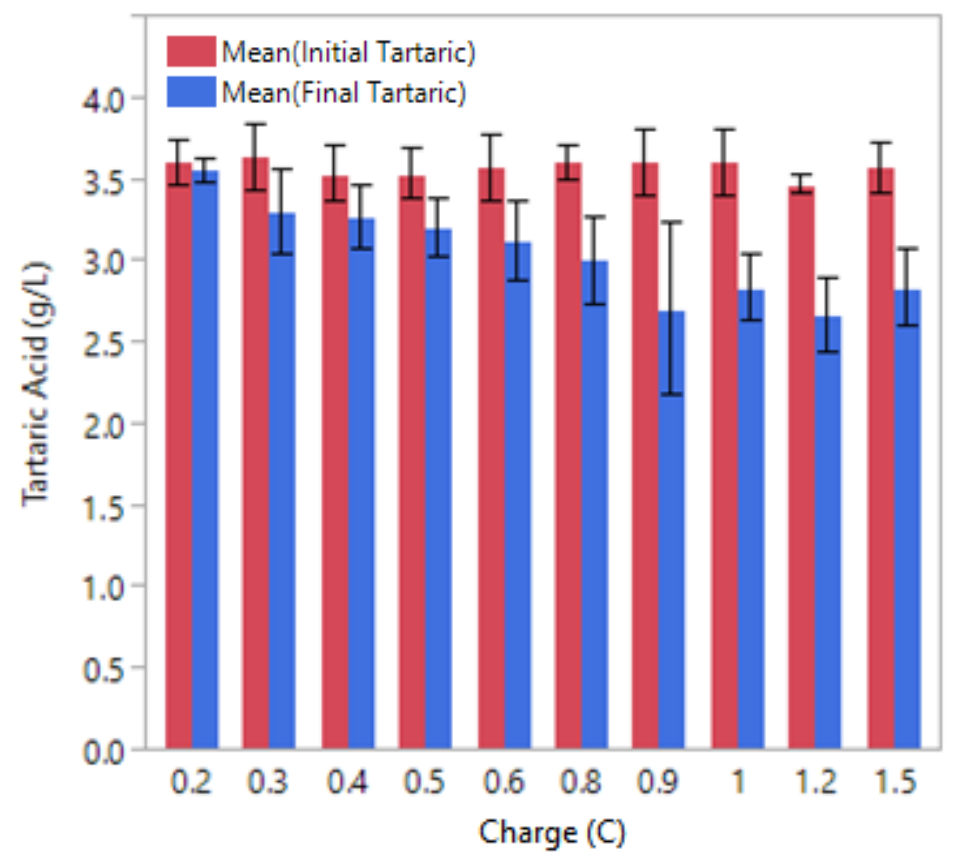

Figure 19. Comparison of the effect of electrical charge (coulombs) on the intial and final tartaric acid concentration for before and after electrolytic treatment at one standard deviation. 


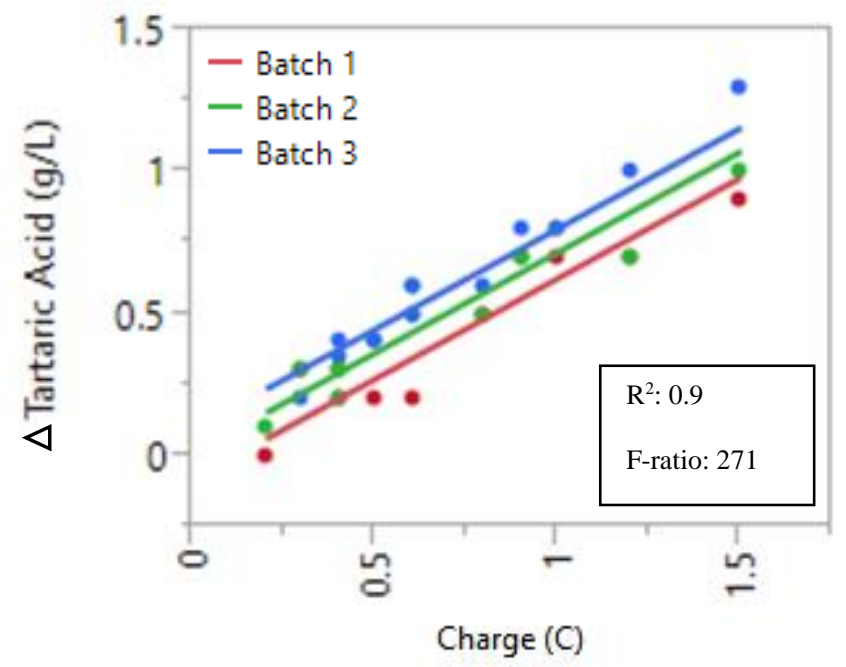

Figure 20. The effect of electrical charge (coulombs) on tartaric acid concentration differences between the initial and electrolytically treated wine.

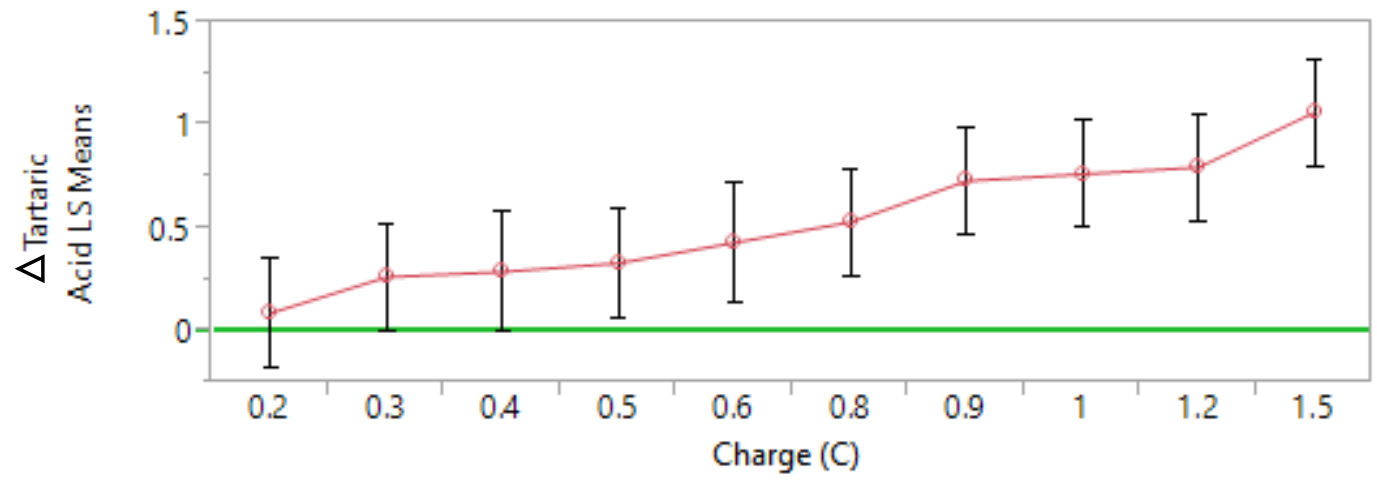

Figure 21. $\Delta$ Tartaric acid least square means for different charge treatments with $99 \%$ confidence interval.

Table 7. Tukey comparison of charge treatments for mean differences in tartaric acid concentration.

\begin{tabular}{|c|c|c|c|c|c|}
\hline Charge & & & & & $\Delta$ Least Sq Mean \\
\hline 1.5 & A & & & & 1.0666667 \\
\hline 1.2 & A & B & & & 0.8000000 \\
\hline 1 & A & B & & & 0.7666667 \\
\hline 0.9 & & B & & & 0.7333333 \\
\hline 0.8 & & B & C & & 0.5333333 \\
\hline 0.6 & & & C & & 0.4333333 \\
\hline 0.5 & & & C & D & 0.3333333 \\
\hline 0.4 & & & C & D & 0.2916667 \\
\hline 0.3 & & & C & D & 0.2666667 \\
\hline 0.2 & & & & D & 0.0910354 \\
\hline
\end{tabular}




\subsection{Effect of the Electrolytic Process on Titratable Acidity Concentration in Wine}

The effect of increasing charge on titratable acidity is shown in Figure 7. The $\Delta$ Titratable acidity is the titratable acidity difference between the initial untreated wine and the final electrolytic treated for each sample, which equals to the decrease of titratable acidity. There does not appear to be a defined linear correlation between charge and the amount of decrease in titratable acidity (Figure 8). Also, in Figure 9, the mean titratable acidity concentration differences for each treatment with $99 \%$ confidence intervals were compared with the baseline initial, where the titratable acidity difference between untreated and treated wines was 0 . With $99 \%$ confidence, all treatment groups showed a significant decrease from the initial titratable acidity concentration, except for 0.2, 0.3, and 0.5 coulombs (Figure 9) which had no significant difference from its initial concentration. The Tukey comparisons for all treatments were done in Table 8 and showed no significant differences between any of the charge treatments.

The decrease in titratable acidity has been observed with electrodialysis treated wines (Bories et al. 2011). The most likely reason for the decrease in titratable acidity is the removal of tartaric acid in the wine, which makes up most of the wine's acidity. Furthermore, the focus was only on tartaric acid because it had been shown in previous studies that malic, lactic, and acetic acids were not significantly affected by electrodialysis or cold treatment (Gómez Benítez et al. 2003). Therefore, it can be inferred that a decrease in tartaric acid concentration is directly related to a decrease in titratable acidity. However, in our study the trend for titratable acidity did not follow the same trend as that of tartaric acid. The belief was that the tartaric acid was converted into dioxosuccinic acid, which is still measureable by titratable acidity (Song et al. 2012). Thus, the total acid content in the wine did not change as initially predicted. 


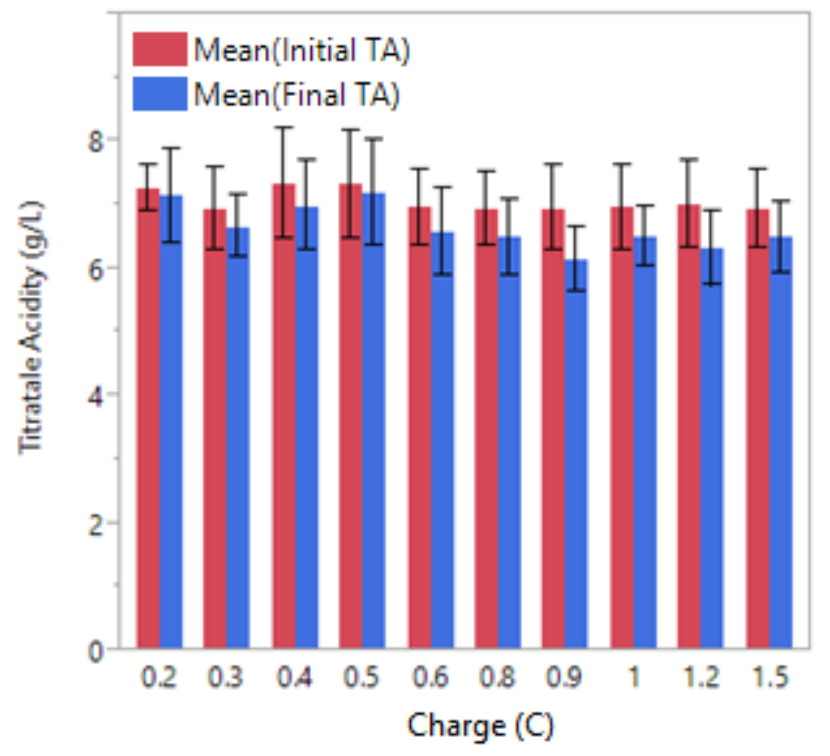

Figure 22. Comparison of the effect of electrical charge (coulombs) on the initial and final titratable acidity concentration for before and after electrolytic treatment at one standard deviation

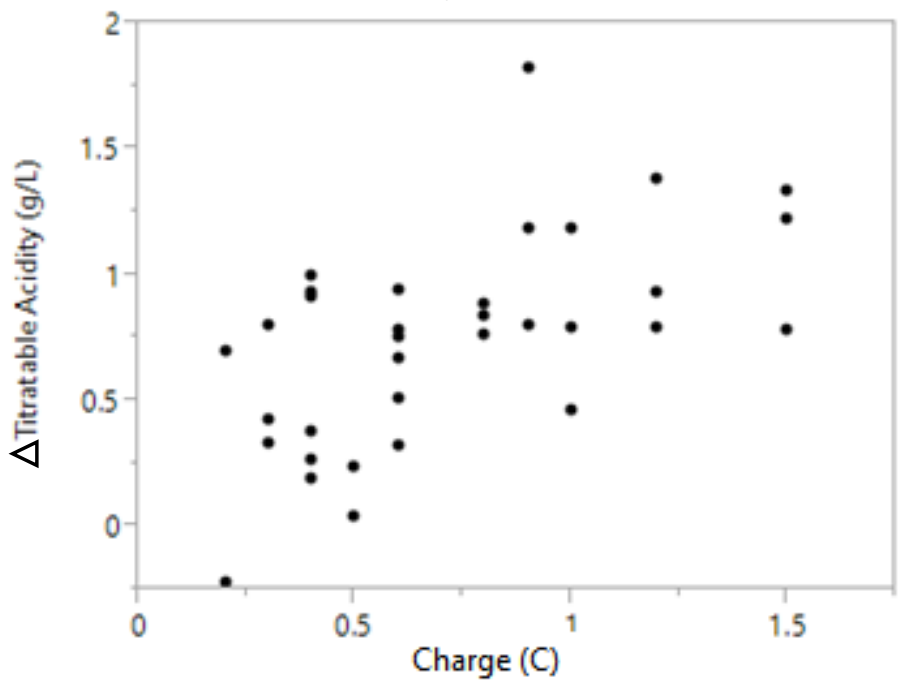

Figure 23. The effect of electrical charge (coulombs) on titratable acidity concentration differences between the initial and electrolytically treated wine. 


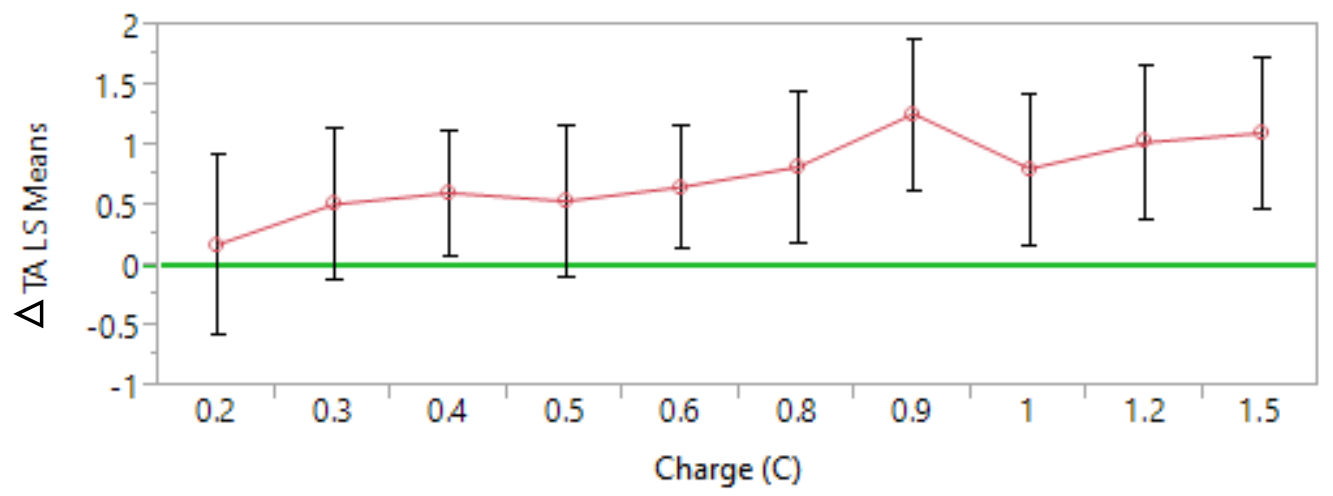

Figure 24. $\Delta$ Titratable acidity least square means for different charge treatments with $99 \%$ confidence interval.

Table 8. Tukey comparisons of charge treatments for mean differences in titratable acidity.

\begin{tabular}{llr}
\hline Charge & & $\Delta$ Least Sq Mean \\
\hline 0.9 & A & 1.2703870 \\
1.5 & A & 1.1112604 \\
1.2 & A & 1.0332000 \\
0.8 & A & 0.8274222 \\
1 & A & 0.8140889 \\
0.6 & A & 0.6618747 \\
0.4 & A & 0.6130327 \\
0.5 & A & 0.5444889 \\
0.3 & A & 0.5185185 \\
0.2 & A & 0.1839267 \\
\hline
\end{tabular}

\subsection{Effect of Electrolytic Process on Wine pH}

The effect of increasing charge on $\mathrm{pH}$ is shown in Figure 10. The $\Delta \mathrm{pH}$ is the $\mathrm{pH}$ difference between the initial untreated wine and the final electrolytic treated for each sample, which equals to the decrease of $\mathrm{pH}$. There is no statistically significant correlation between charge and differences in $\mathrm{pH}$ (Figure 11). In Figure 12, the mean $\mathrm{pH}$ differences for each treatment with $99 \%$ confidence intervals were compared with the baseline initial, where the $\mathrm{pH}$ difference between untreated and treated wines was 0 . All electrolytic treated wines were not significantly different from the initial wine $\mathrm{pH}$ because all treatment confidence intervals included the baseline value (Figure 12). The Tukey comparisons for all treatments were done in Table 9 to show any significant 
differences between charge treatments. There was no statistically significant difference between any of the charge treatments for mean differences in $\mathrm{pH}$ (Table 9).

Typically in past studies, the $\mathrm{pH}$ tends to drop slightly between 0.1 and 0.2 for the electrodialysis treated wines (STARS 2011). Cold stabilized wines also drop in $\mathrm{pH}$ to a larger degree than electrodialysis due to the increase in tartaric acid crystals added for the cold stability process. The disruption to the tartrate equilibrium causes $\mathrm{H}_{2} \mathrm{~T}$ to convert to $\mathrm{HT}^{-}$release an $\mathrm{H}^{+}$, which increases the acidity (Lasanta and Gómez 2012). However, in this study, the $\mathrm{pH}$ change would more likely be the opposite of previous literature values. If any changes in $\mathrm{pH}$ were to happen it would most likely increase or have no change in $\mathrm{pH}$ due to the sole reduction or conversion of tartaric acid with no external addition of any $\mathrm{H}+$ or acids.

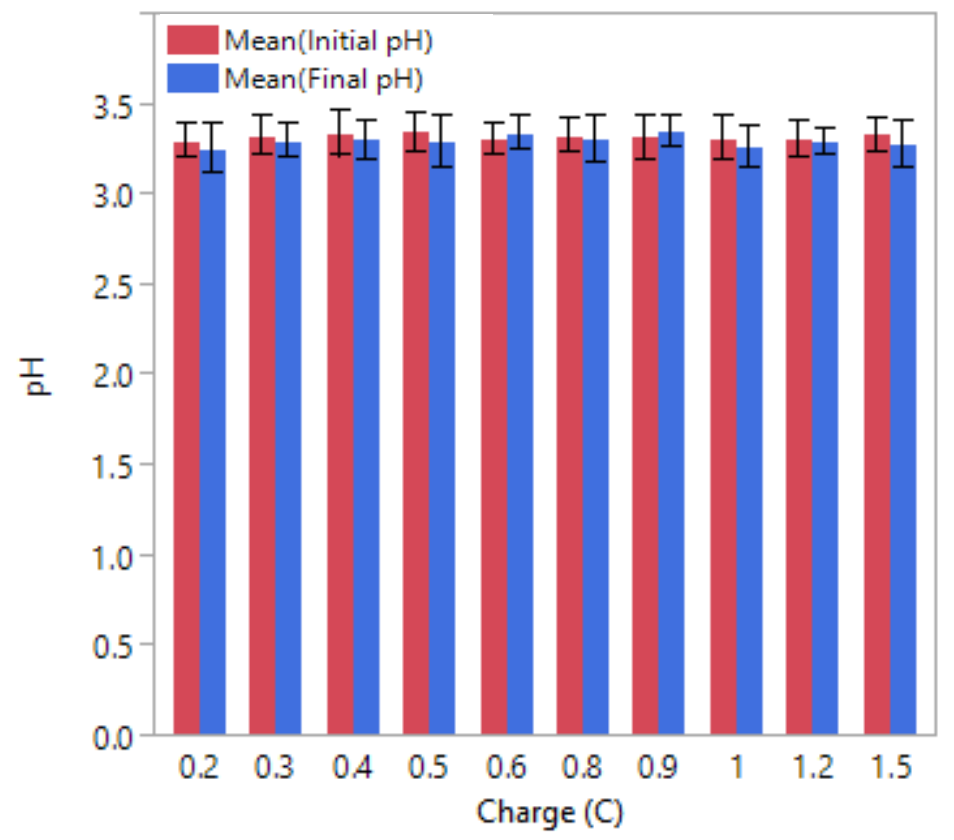

Figure 25. Comparison of the effect of electrical charge (coulombs) on the intial and final pH for before and after electrolytic treatment at one standard deviation. 


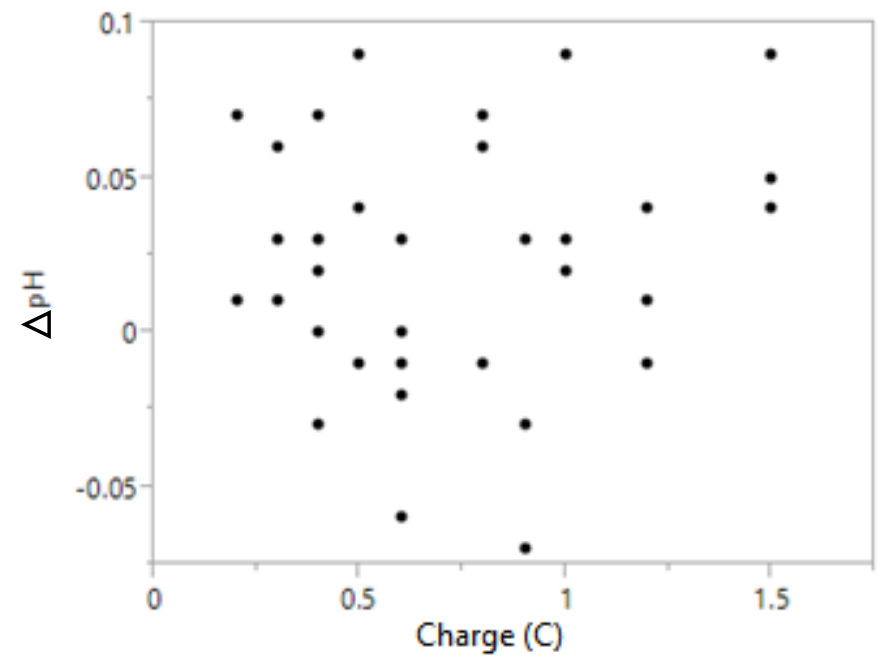

Figure 26. The effect of electrical charge (coulombs) on pH differences between the initial and electrolytically treated wine.

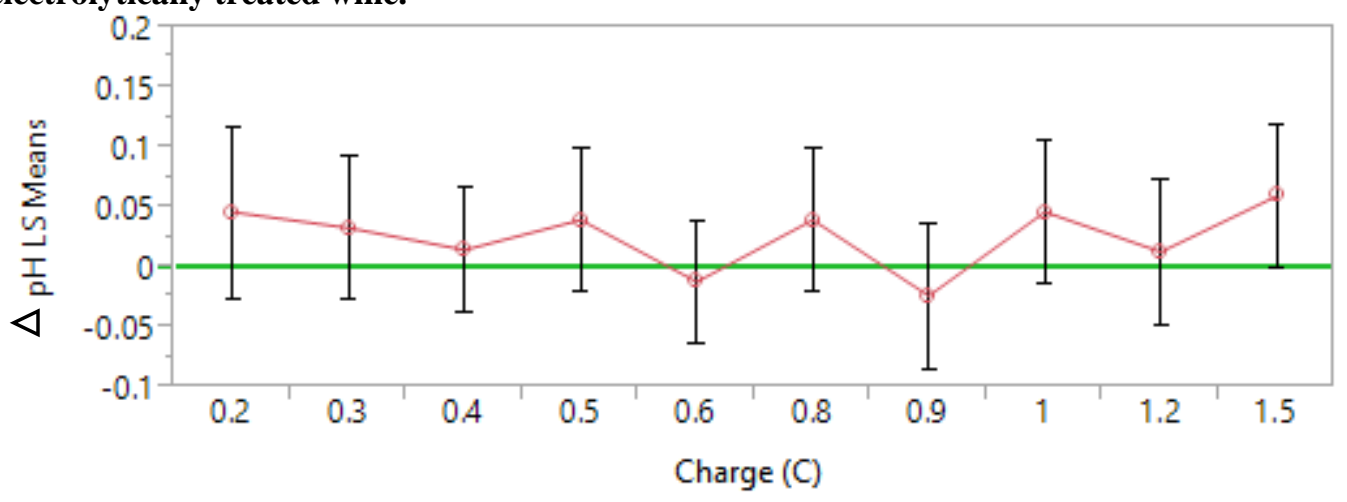

Figure 27. $\Delta \mathrm{pH}$ least square means for different charge treatments with $99 \%$ confidence interval.

Table 9. Tukey comparison of charge treatments for mean differences in $\mathrm{pH}$.

\begin{tabular}{llr}
\hline Charge & & $\boldsymbol{\Delta L}$ Least Sq Mean \\
\hline 1.5 & A & 0.0600000 \\
1 & A & 0.0466667 \\
0.2 & A & 0.0464918 \\
0.5 & A & 0.0400000 \\
0.8 & A & 0.0400000 \\
0.3 & A & 0.0333333 \\
0.4 & A & 0.0150000 \\
1.2 & A & 0.0133333 \\
0.6 & A & -0.0116667 \\
0.9 & A & -0.0233333 \\
\hline
\end{tabular}




\subsection{Effect of Electrolytic Process on the Color of Wine}

\subsubsection{Effect of Electrolytic Process on the Hue Value of Wine}

The effect of increasing charge on hue is shown in Figure 28. The $\Delta$ Hue is the hue difference between the initial untreated wine and the final electrolytic treated for each sample, which equals to the decrease of hue. There does not appear to be a significant linear correlation between charge and the difference in hue after treatment (Figure 29). In Figure 30, the mean hue differences for each treatment with 99\% confidence intervals were compared with the baseline initial, no differences. All electrolytic treated wines were all significantly different from the initial wine's hue except for 0.2 coulomb treatment (Figure 30). The Tukey comparisons for all treatments were done in Table 10 to show any significant differences between charge treatments for hue. None of the hue of the cold stabilized wines were significantly different from each other (Table 10).

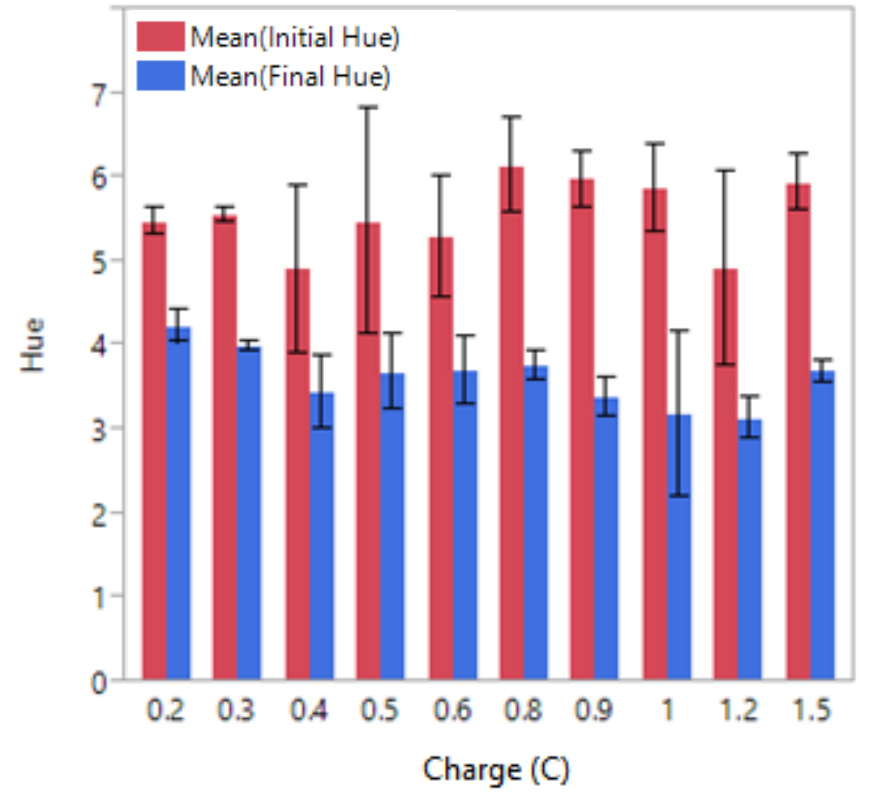

Figure 28. Comparison of the effect of electrical charge (coulombs) on the intial hue for before and after electrolytic treatment at one standard deviation. 


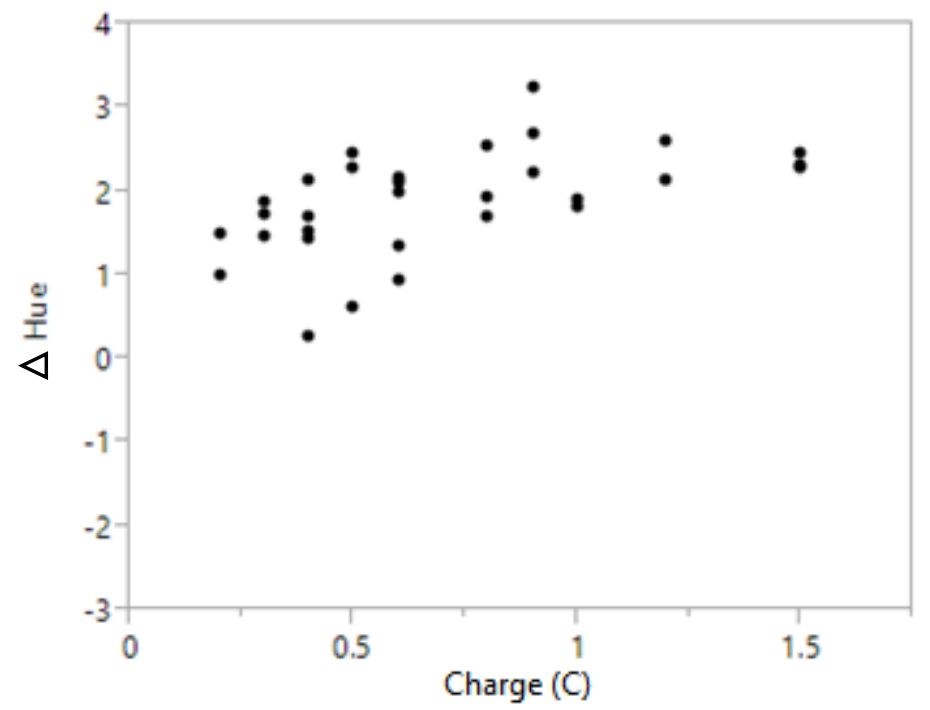

Figure 29. The effect of electrical charge (coulombs) on hue differences between the initial and electrolytic treated wine.

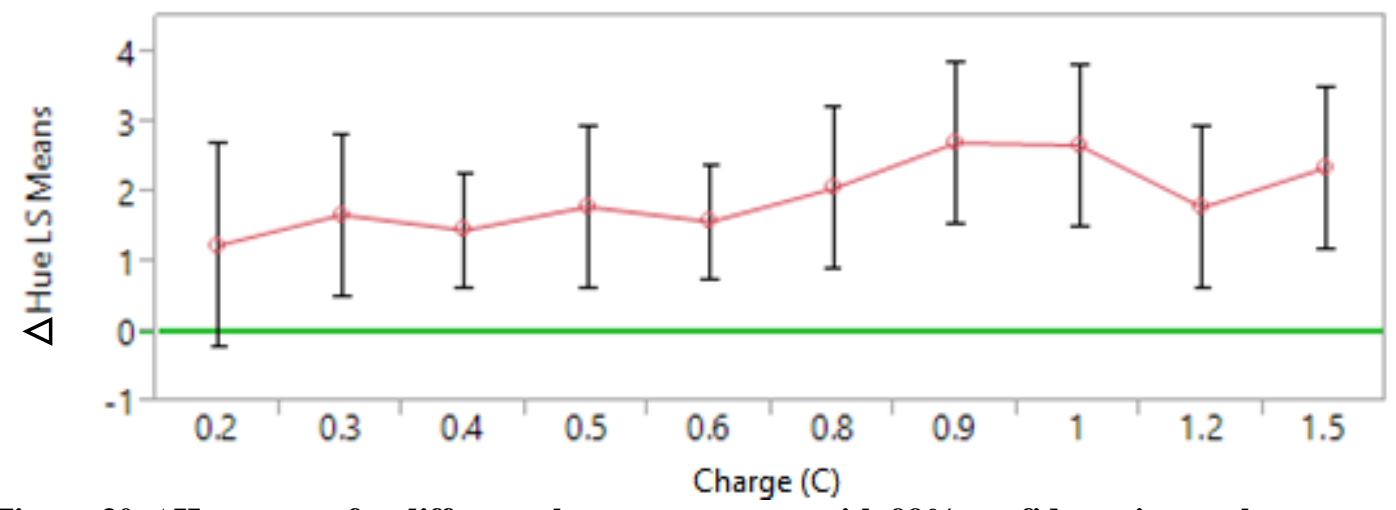

Figure 30. $\Delta$ Hue means for different charge treatments with $99 \%$ confidence interval.

Table 10. Tukey comparison of charge treatments for mean differences in hue.

\begin{tabular}{llc}
\hline Charge & & $\Delta$ Least Sq Mean \\
\hline 0.9 & A & 2.7073520 \\
1 & A & 2.6706177 \\
1.5 & A & 2.3496803 \\
0.8 & A & 2.0551387 \\
0.5 & A & 1.7923988 \\
1.2 & A & 1.7841057 \\
0.3 & A & 1.6798353 \\
0.6 & A & 1.5800383 \\
0.4 & A & 1.4566261 \\
0.2 & A & 1.2428445 \\
\hline
\end{tabular}




\subsubsection{Effect of Electrolytic Process on the Intensity Value of Wine}

The effect of increasing charge on intensity is shown in Figure 31 . The $\Delta$ Intensity is the intensity difference between the initial untreated wine and the final electrolytic treated for each sample, which equals to the decrease of intensity. There is no linear correlation between charge and the difference in intensity after treatment (Figure 32). In Figure 33, the mean intensity differences for each treatment with $99 \%$ confidence intervals were compared with the baseline initial, where the intensity difference between untreated and treated wines was 0 . None of the treatments were significantly different from the initial wine's intensity because all treatment confidence intervals contained the baseline value (Figure 33). The Tukey comparisons for all treatments were done in Table 11 to show any significant differences between charge treatments. There was no statistically significant difference between any of the charge groups with each other (Table 11)

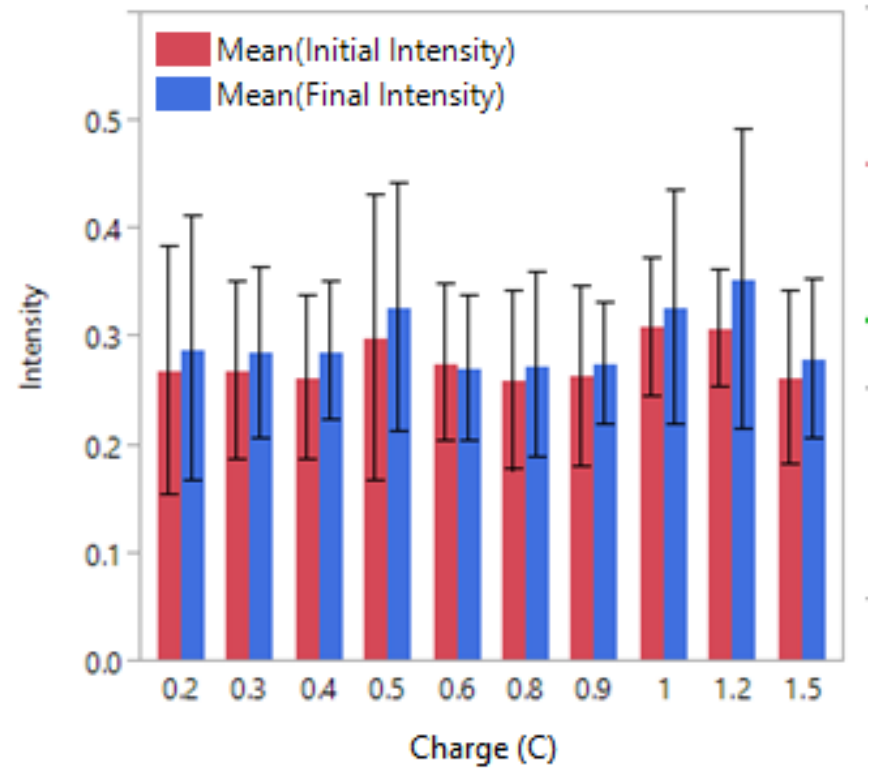

Figure 31. Comparison of the effect of electrical charge (coulombs) on the intial and final Intensity for before and after electrolytic treatment at one standard deviation. 


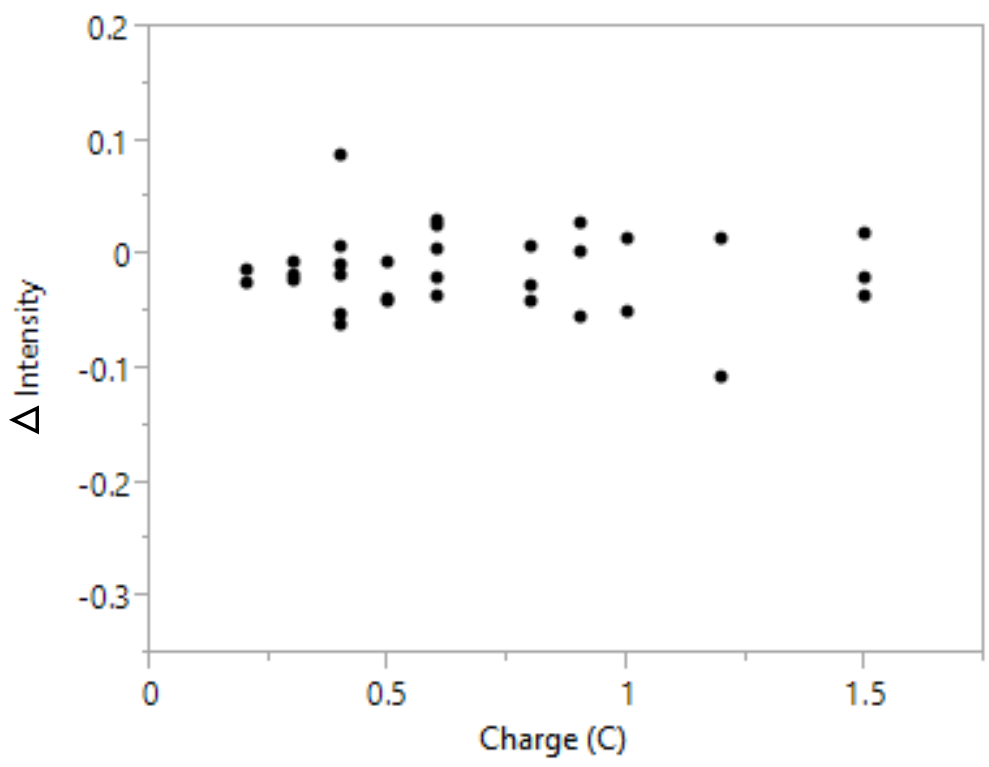

Figure 32. The effect of electrical charge (coulombs) on intensity differences between the initial and electrolytic treated wine.

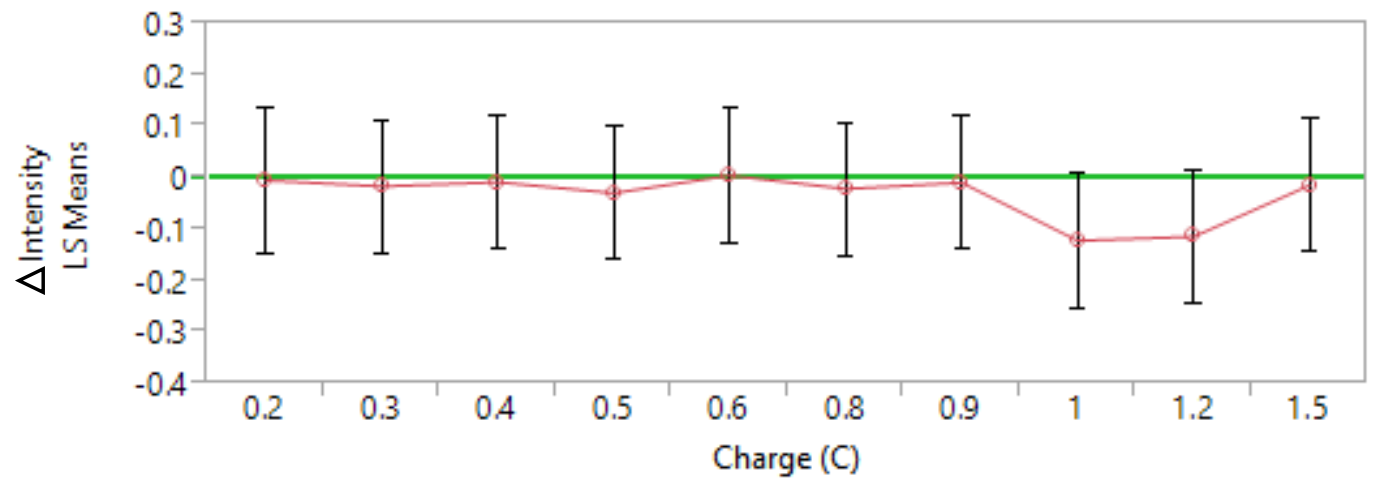

Figure 33. $\Delta$ Intensity least square means for different charge treatments with $99 \%$ confidence interval.

Table 11. Tukey comparison of charge treatments for mean differences in intensity.

\begin{tabular}{llc}
\hline Charge & & $\boldsymbol{\Delta L e a s t ~ S q ~ M e a n ~}$ \\
\hline 0.6 & A & 0.0058333 \\
0.2 & A & -0.0036697 \\
0.4 & A & -0.0071667 \\
0.9 & A & -0.0080000 \\
1.5 & A & -0.0130000 \\
0.3 & A & -0.0153333 \\
0.8 & A & -0.0200000 \\
0.5 & A & -0.0283333 \\
1.2 & A & -0.1130000 \\
1 & A & -0.1210000 \\
\hline
\end{tabular}


Typically, there has been no significant change in the color after cold or electrodialysis treatment (Santos et al. 2002; Bories et al. 2011). The intensity results agreed with previous literature findings of having no significant differences between the initial and treated wine. However, it was a little surprising that in our study there were significant differences in the hue of the wine after treatment when compared to the initial. A possibility is that polyphenols were oxidized at the anode, which commonly leads to browning (Oliveira et al. 2011). The lower hue values agreed with the browning assumption. In addition, the electrolytic process increases the temperature and exposure to oxygen are known to lead to rapid oxidative browning, which typically negatively affects sensory characteristics (Dharmadhikari 2015). A darker hue does not necessarily mean the flavor of the wine has decreased in any sense, because darker white wines are known and expected to be more mature than pale white wines (Parr et al. 2003).

Through personal observation, it was noted that color of the wine appeared to be darker after electrolytic treatment (Figure 34). According to previous findings, no noticeable changes in color were found until the wine was heated past $50{ }^{\circ} \mathrm{C}$ (Dias et al. 2012). In this study, the hue decreased for all electrolytic treatments which were all below $50{ }^{\circ} \mathrm{C}$. Another potential cause of the wine hue becoming darker in appearance may be due to the graphite electrodes shedding some of its carbon into the wine solution.

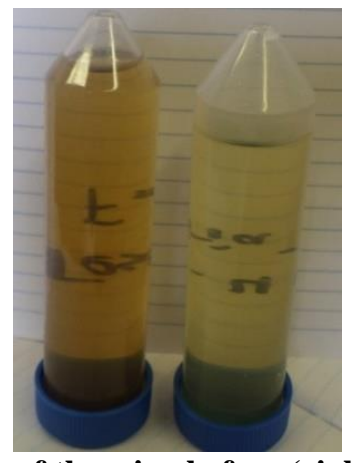

Figure 34. Observational color changes of the wine before (right) and after (left) electrolytic treatment. 


\subsection{Effect of the Electrolytic Process on Mineral (K, Ca, Mg, Na) Reduction in Wine}

The effect of increasing charge on mineral composition is shown in Figure 35, 36, 37, 38. There is an increasing trend for $\mathrm{K}, \mathrm{Mg}$, and Ca removal with increasing charge (Figure 39, 40, 41). However, for sodium there was no correlation with charge (Figure 42). At certain charges, the $\mathrm{K}, \mathrm{Mg}$, and $\mathrm{Ca}$ were significantly different from its initial mineral composition (Figure 43, 44, 45). Again, sodium was not found to have any significant differences in concentration compared to its initial after electrolytic treatment (Figure 46).

The effect of charge on the mean potassium concentration before and after electrolytic treatment is shown in Figure 35 . The $\Delta$ Potassium was the potassium concentration difference between the initial untreated wine and the final electrolytic treated for each sample, which equals to the decrease of potassium. There is a lack of linear correlation between charge and the difference in potassium after treatment (Figure 39), but the data suggests that as charge increases so does the removal of potassium. In Figure 43, the mean potassium differences for each treatment with $99 \%$ confidence intervals were compared with the baseline initial, where the potassium difference between untreated wines was 0 . All electrolytic treatments except $0.2,0.3,0.4$, and 0.6 coulombs removed enough potassium to significantly differentiate it from the initial concentration (Figure 43). The Tukey comparisons for all treatments were done in Table 12 to show any significant differences between electrolytic treated groups for potassium.

The effect of charge on the mean calcium concentration before and after electrolytic treatment is shown in Figure 36 . The $\Delta$ Calcium was the calcium concentration difference between the initial untreated wine and the final electrolytic treated for each sample, which equals to the decrease of calcium. There appears to be a 
linear correlation between charge and the difference in calcium after treatment (Figure 40), whereas charge increased so did the removal of calcium. In Figure 44, the mean calcium differences for each treatment with 99\% confidence intervals were compared with the baseline initial, where the calcium difference between untreated wines was 0 . All treatments except $0.2,0.3,0.4,0.6$ coulombs were significantly different from the initial (Figure 44). The electrolytic treatments effect on calcium was similar to that of potassium. The Tukey comparisons for all treatments were done in Table 13 to show any significant differences between electrolytic treated groups for calcium.

The effect of charge on the mean magnesium concentration before and after electrolytic treatment is shown in Figure 37. The $\Delta$ Magnesium was the magnesium concentration difference between the initial untreated wine and the final electrolytic treated for each sample, which equals to the decrease of magnesium. There is a lack of linear correlation between charge and the difference in magnesium after treatment (Figure 41), but it appeared that as charge increased so did the removal of magnesium. In Figure 45, the mean magnesium differences for each treatment with $99 \%$ confidence intervals were compared with the baseline initial, where the magnesium difference between untreated wines was 0 . Treatments with 0.8 or more coulombs were significantly different from initial by decreasing the magnesium concentration (Figure 45). The Tukey comparisons for all treatments were done in Table 14 to show any significant differences between electrolytic treated groups for magnesium.

The effect of charge on the mean sodium concentration before and after electrolytic treatment is shown in Figure 38 . The $\Delta$ Sodium was the sodium concentration difference between the initial untreated wine and the final electrolytic treated for each 
sample, which equals to the decrease of sodium. There is a lack of correlation between charge and the difference in sodium after treatment (Figure 42). In Figure 46, the mean sodium differences for each treatment with $99 \%$ confidence intervals were compared with the baseline initial, where the sodium difference between untreated wines was 0 . All treatments were not different from the initial because the confidence intervals included the baseline value of 0 (Figure 46). The Tukey comparisons for all treatments were done in Table 15 to show any significant differences between electrolytic treated groups for sodium. There was no significant differences between any of the charge groups for sodium concentration.

These results were comparable to the data previously reported in the literature for electrodialysis treated wines, where $\mathrm{K}, \mathrm{Mg}$, and $\mathrm{Ca}$ were reduced and $\mathrm{Na}$ had no significant reduction (Santos et al. 2002; Bories et al. 2011; Lasanta and Gómez 2012). The percent reduction of $\mathrm{Ca}$ was often larger than $\mathrm{K}$ after our electrolytic treatment by about 3 times, even though the actual mass of Ca removed was less than that of $\mathrm{K}$. While the concentration of $\mathrm{K}$ is almost 10 times more than $\mathrm{Ca}$, calcium is more electronegative than potassium and is more mobile in solution. Verification and confirmation of these minerals being removed onto the electrodes was performed by analyzing the wash water for these minerals. Unlike electrodialysis, cold treated wines are known to only reduce $\mathrm{K}$ and not Mg and $\mathrm{Ca}$ (Lasanta and Gómez 2012). These minerals have not been officially linked to significantly affecting the sensory characteristics, except for sodium, which contributes to a salty flavor (Cabello-Pasini et al. 2013). In our study, Na was not significantly affected so it can be assumed that there were not changes in flavor due to sodium content. 


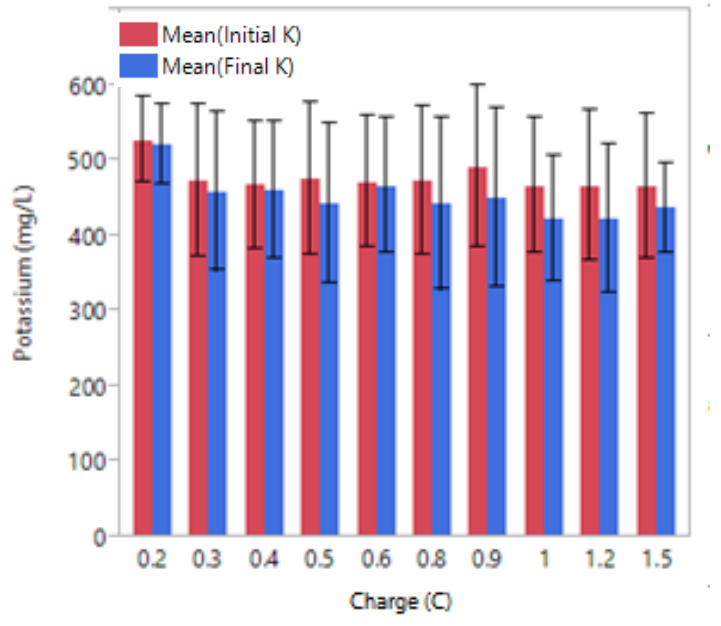

Figure 35. Comparison of the effect of electrical charge (coulombs) on the initial and final potassium concentration for before and after electrolytic treatment at one standard deviation.

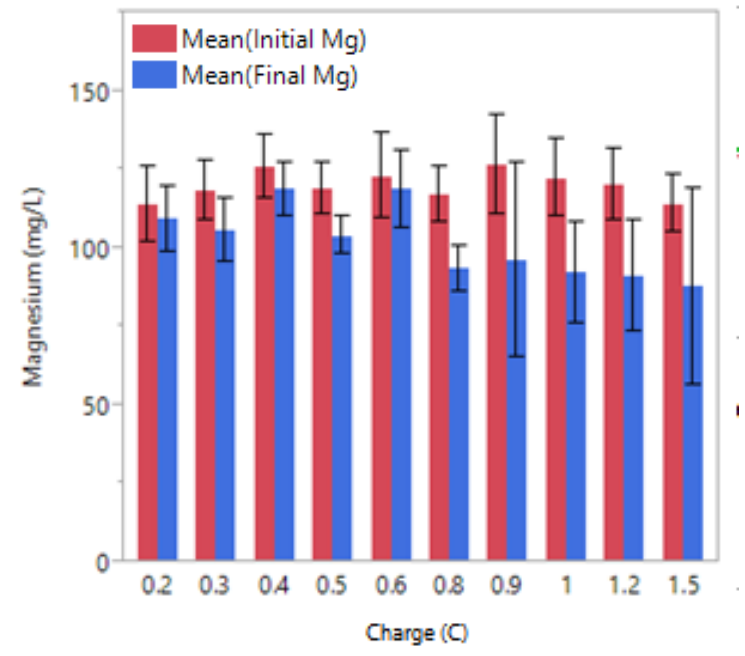

Figure 37. Comparison of the effect of electrical charge (coulombs) on the intial and final magnesium concentration for before and after electrolytic treatment at one standard deviation.

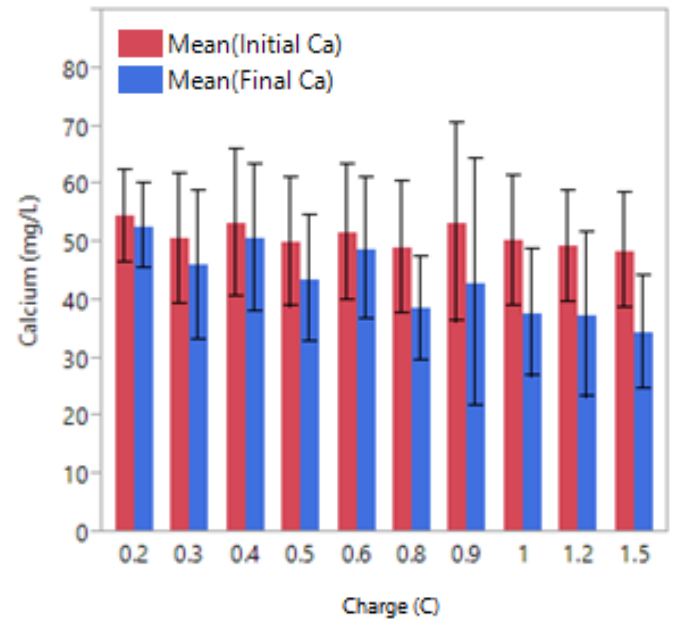

Figure 36. Comparison of the effect of electrical charge (coulombs) on the initial and final calcium concentration for before and after electrolytic treatment at one standard deviation.

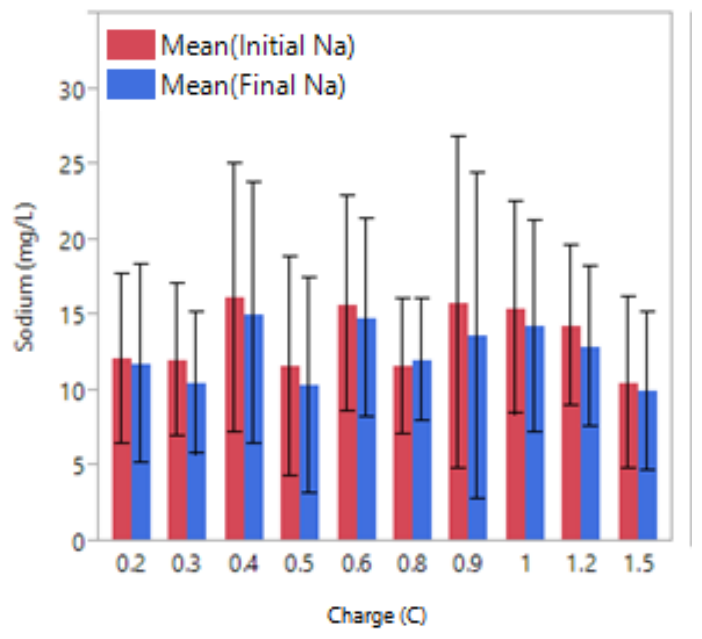

Figure 38. Comparison of the effect of electrical charge (coulombs) on the intial and final sodium concentration for before and after electrolytic treatment at one standard deviation. 


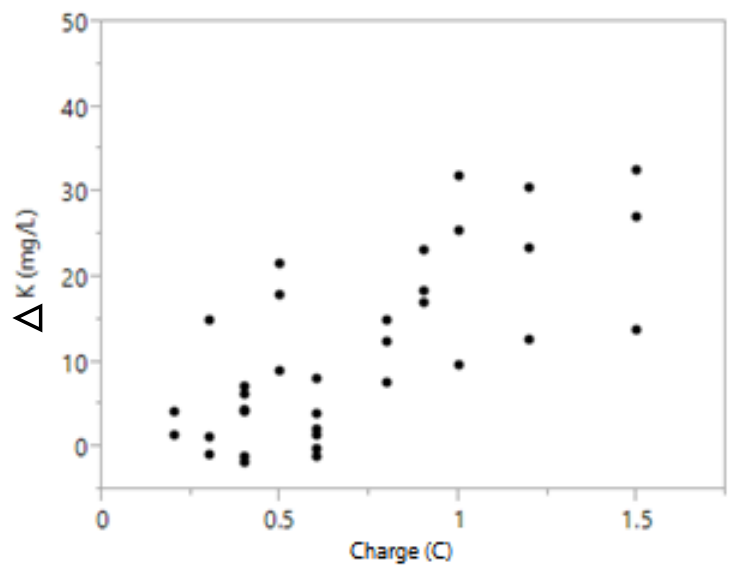

Figure 39. The effect of electrical charge (coulombs) on $\mathrm{K}$ differences between the initial and electrolytically treated wine.

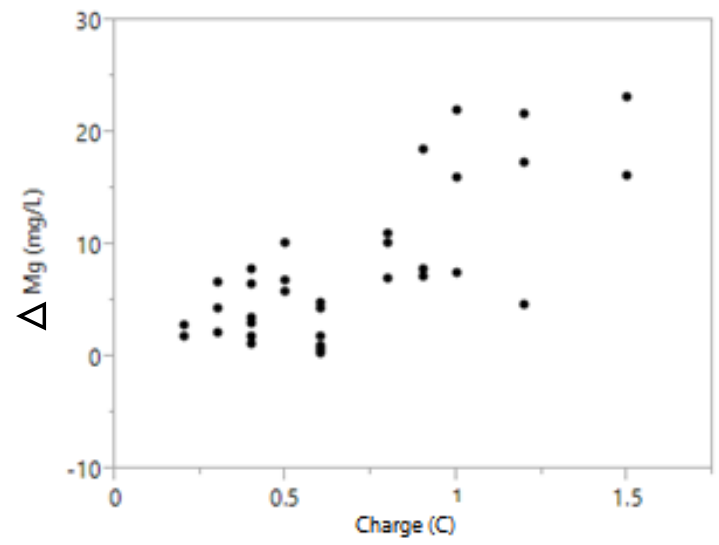

Figure 41. The effect of electrical charge (coulombs) on Mg differences between the initial and electrolytically treated wine.

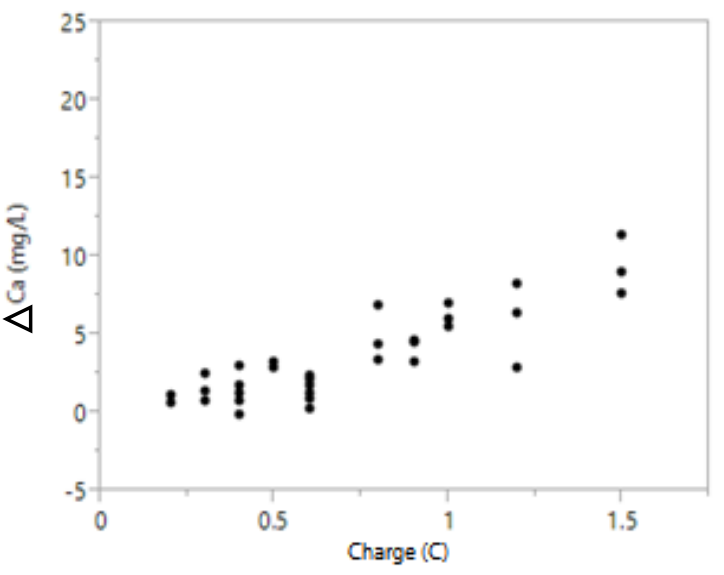

Figure 40 The effect of electrical charge (coulombs) on Ca differences between the initial and electrolytically treated wine

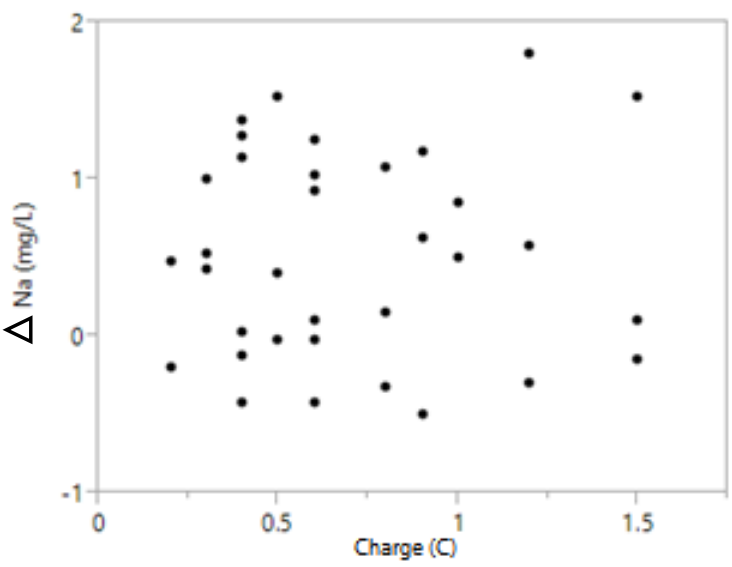

Figure 42. The effect of electrical charge (coulombs) on $\mathrm{Na}$ differences between the initial and electrolytically treated wine. 


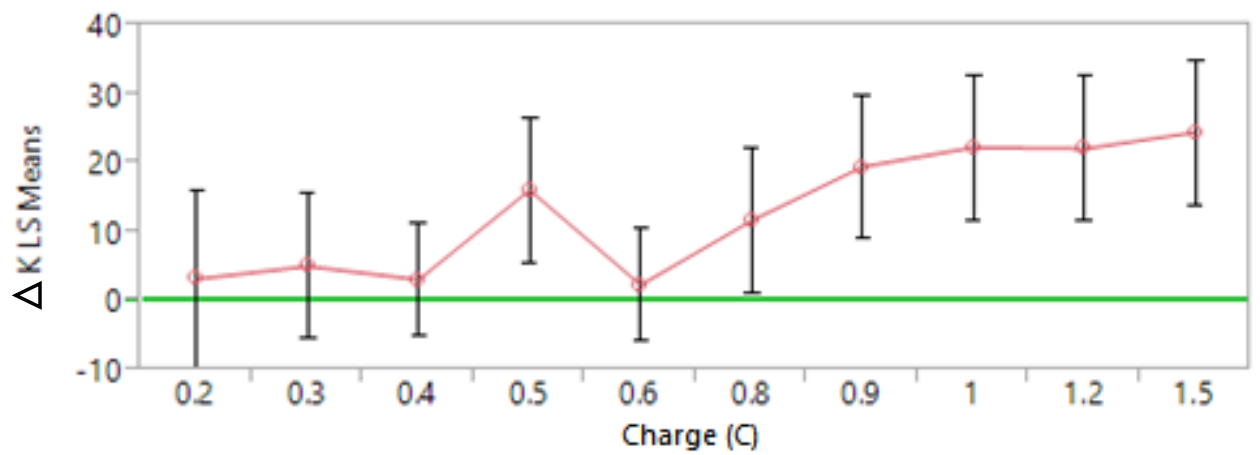

Figure 43. $\Delta \mathrm{K}$ means for different charge treatments with $99 \%$ confidence interval.

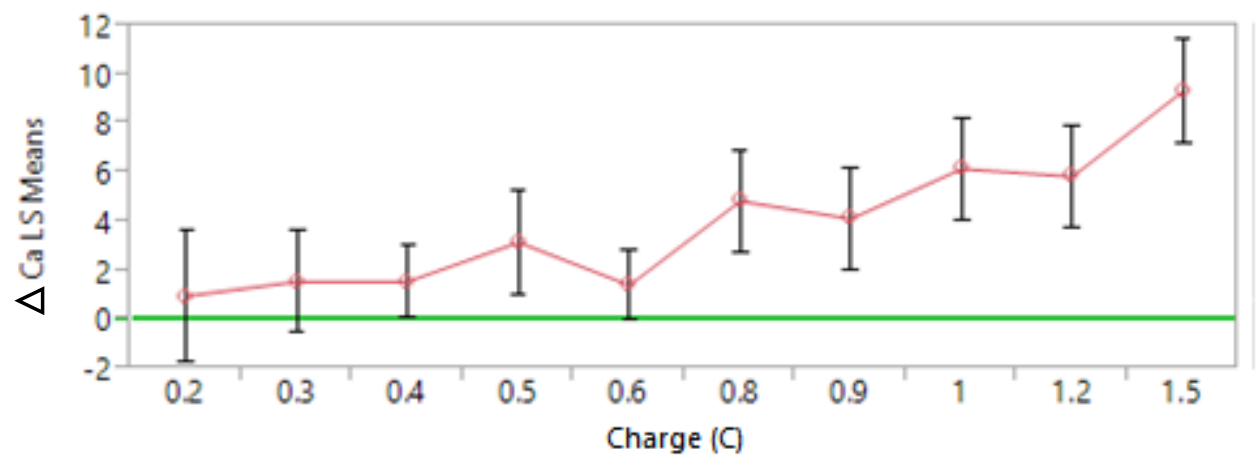

Figure 44. $\triangle$ Ca means for different charge treatments with $99 \%$ confidence interval.

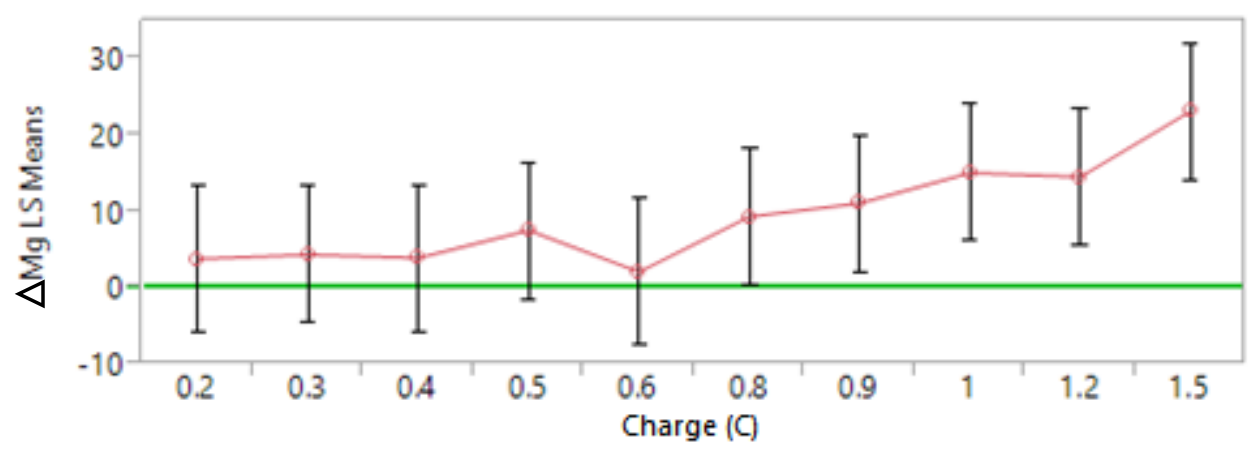

Figure 45. $\Delta \mathrm{Mg}$ means for different charge treatments with $99 \%$ confidence interval.

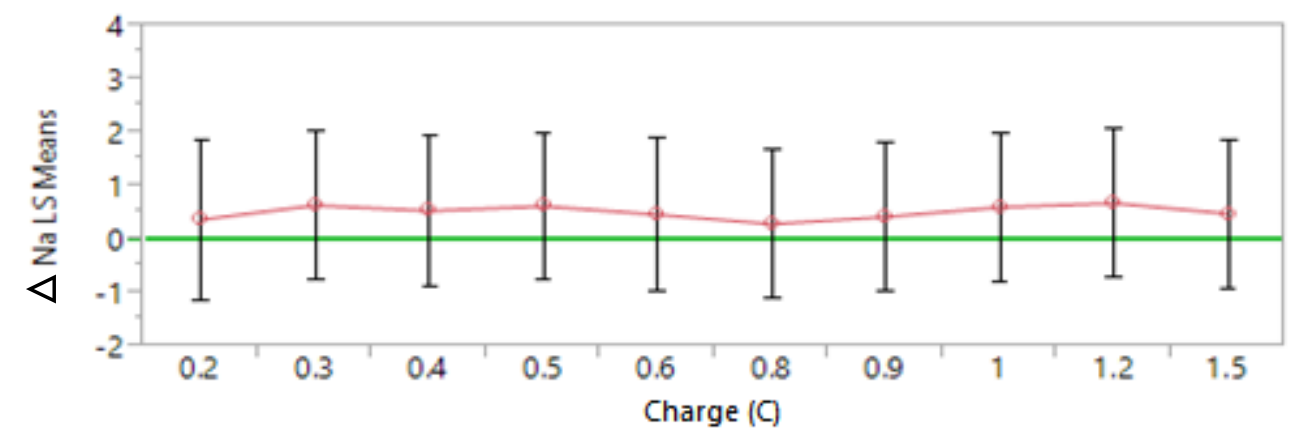

Figure 46. $\triangle \mathrm{Na}$ means for different charge treatments with $99 \%$ confidence interval. 
Table 12. Tukey comparison of charge treatments for mean differences in potassium

\begin{tabular}{|c|c|c|c|}
\hline Charge & & & $\Delta$ Least Sq Mean \\
\hline 1.5 & $A$ & & 24.516667 \\
\hline 1 & A & & 22.341667 \\
\hline 1.2 & A & & 22.150000 \\
\hline 0.9 & A & B & 19.508333 \\
\hline 0.5 & A & B & 16.175000 \\
\hline 0.8 & A & B & 11.716667 \\
\hline 0.3 & A & B & 5.116667 \\
\hline 0.2 & A & B & 3.262563 \\
\hline 0.4 & & B & 3.170833 \\
\hline 0.6 & & B & 2.387500 \\
\hline
\end{tabular}

Table 13. Tukey comparison of charge treatments for mean difference in calcium

\begin{tabular}{|c|c|c|c|c|c|}
\hline Charge & & & & & $\Delta$ Least Sq Mean \\
\hline 1.5 & A & & & & 9.3500000 \\
\hline 1 & A & B & & & 6.1750000 \\
\hline 1.2 & A & B & C & & 5.8666667 \\
\hline 0.8 & A & B & C & D & 4.8583333 \\
\hline 0.9 & & B & C & D & 4.1416667 \\
\hline 0.5 & & B & C & D & 3.1750000 \\
\hline 0.4 & & & & D & 1.5791667 \\
\hline 0.3 & & & C & D & 1.5750000 \\
\hline 0.6 & & & & D & 1.4291667 \\
\hline 0.2 & & & C & D & 0.9804230 \\
\hline
\end{tabular}

Table 14. Tukey comparison of charge treatments for mean differences in magnesium

\begin{tabular}{|c|c|c|c|c|c|}
\hline Charge & & & & & $\Delta$ Least Sq Mean \\
\hline 1.5 & A & & & & 23.233333 \\
\hline 1 & A & B & & & 15.141667 \\
\hline 1.2 & A & B & C & & 14.608333 \\
\hline 0.9 & A & B & C & D & 11.141667 \\
\hline 0.8 & & B & C & D & 9.400000 \\
\hline 0.5 & & $B$ & C & D & 7.591667 \\
\hline 0.3 & & B & C & D & 4.391667 \\
\hline 0.4 & & & C & D & 3.977083 \\
\hline 0.2 & & B & C & D & 3.849462 \\
\hline 0.6 & & & & D & 2.133333 \\
\hline
\end{tabular}

Table 15. Tukey comparison of charge treatments for mean differences in sodium

\begin{tabular}{llr}
\hline Charge & & DLeast Sq Mean \\
\hline 1.2 & A & 0.69166667 \\
0.3 & A & 0.65000000 \\
0.5 & A & 0.63333333 \\
1 & A & 0.61666667 \\
0.4 & A & 0.54375000 \\
1.5 & A & 0.49166667 \\
0.6 & A & 0.47500000 \\
0.9 & A & 0.43333333 \\
0.2 & A & 0.38054796 \\
0.8 & A & 0.30000000 \\
\hline
\end{tabular}

\subsection{Comparing Stability Percent Differences of Initial Untreated Wine to Electrolytic Wine}

The average initial conductivity percent differences and the average final conductivity percent differences for all treatments were compared in Figure 47 to the 5\% conductivity difference stability standard. All mean differences in percent conductivity were lower for wines after electrolytic treatment and cold treatment than the initial. Any percent difference of conductivity that is below the $5 \%$ standard stability line is considered to be tartaric acid stabilized (Figure 47). The lower the mean percent difference in conductivity the more stable the wine is. Looking at the results, it suggests that there could potentially be a trend that more charge leads to lower percent differences 
in the final wine, making them more stable. Anything above 0.6 coulombs appears to be more stable than treatments that are 0.6 coulombs and below (Figure 47).

The conductivity measures the ions in wine solution, mainly potassium ions. Potassium plays an important role in creating the instability and crystallization of KHT, therefore by measuring the conductivity the stability of the wine can be determined (Zoecklein et al. 1990; Bories et al. 2011). With this fact, conductivity was assumed to have followed the same trend as potassium. However, the trend for conductivity did not clearly follow the potassium trend where the conductivity should decrease at a similar rate to potassium decrease. According to previous studies, electrodialyzed wines have shown to stabilize wines and achieved greater stability the longer it was processed (Soares et al. 2009; Lasanta and Gómez 2012). Therefore, it was quite surprising that there were no statistically significant results found. An explanation is that our study focuses purely on the difference in percentages, which had large variability due to using different batches of wine and a relatively small sample size. Our study only concentrated on the percent difference and not whether or not the stability tests concluded a change in stability status. Overall, our study had all but two originally unstable wines become stable after electrolytic treatment according to the $5 \%$ standard. The expectations were to see a similar trend in stability like electrodialysis treated wines as charge increased. The results were pointed in that same direction, but were not as conclusive. 


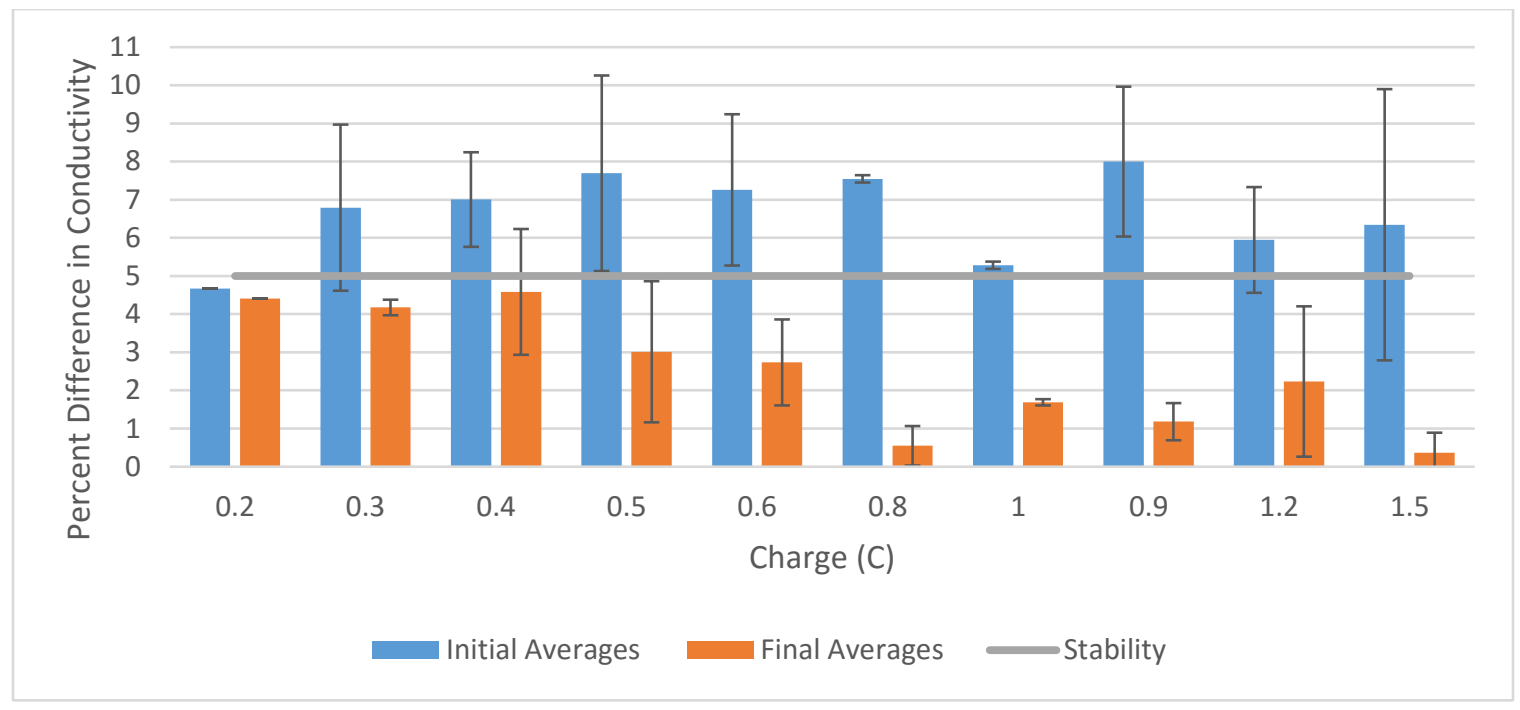

Figure 47. Average initial conductivity percent differences and the average final conductivity percent differences for all treatments within one standard deviation compared with the $5 \%$ difference stability standard.

\subsection{Effect of Electrolytic Process on Chemical Oxygen Demand (COD) Reduction}

The effect of increasing charge on differences in COD is shown in Figure 48. The $\triangle \mathrm{COD}$ was the amount of COD found in the wash water after cleaning the plates from the electrolytic process for each treatment run, which equates to the decrease of COD. There was no statistically significant linear correlation between charge and removal of COD (Figure 48). In Figure 49, the mean COD concentration differences for each treatment with 99\% confidence intervals were compared with the baseline initial, where the COD difference between untreated and treated wines was 0 . There was a statistically significant decrease in COD matter after treatment, and all treatments were different from the initial (Figure 49). According to Table 16, the Tukey comparisons for the charge treatment groups were not significantly different from each other. 


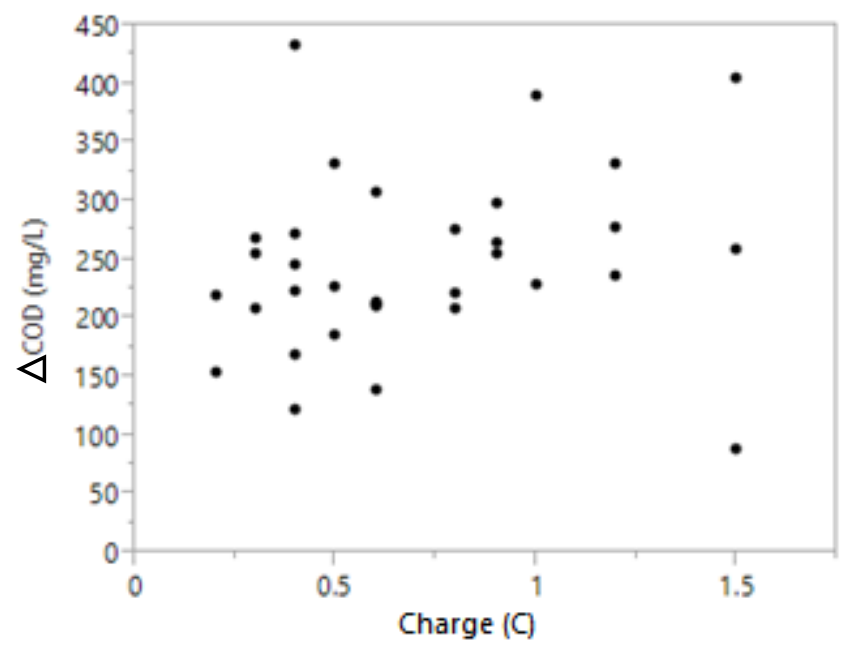

Figure 48. The effect of electrical charge (coulombs) on COD differences between the initial and electrolytic treated wine.

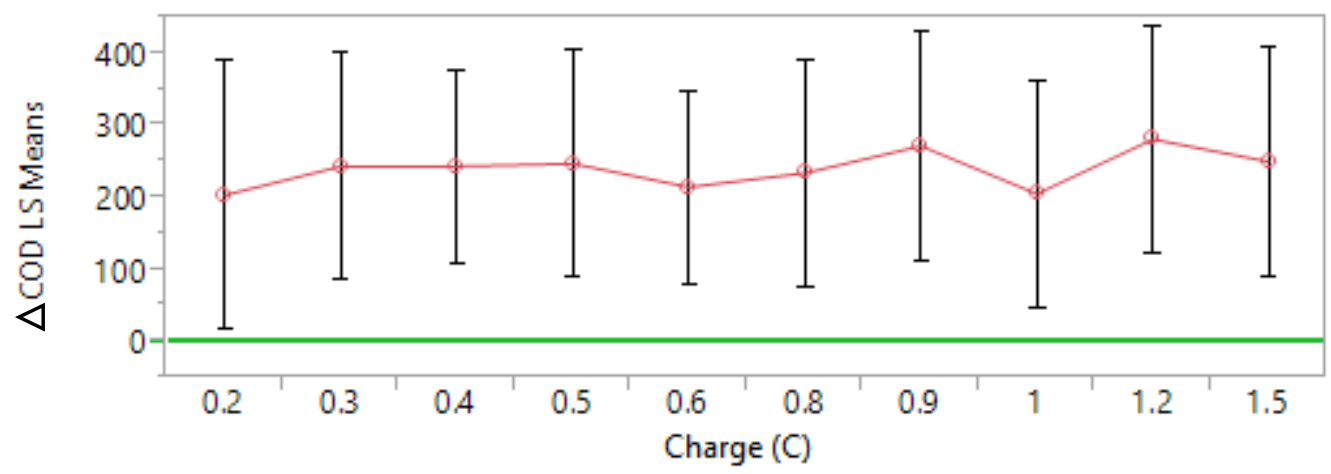

Figure 49. $\triangle$ COD means for different charge treatments with $99 \%$ confidence interval.

Table 16. Tukey comparison of charge treatments for mean differences in COD.

\begin{tabular}{llr}
\hline Charge & & DLeast Sq Mean \\
\hline 1.2 & A & 281.75923 \\
0.9 & A & 272.47680 \\
1.5 & A & 250.72230 \\
0.5 & A & 248.10127 \\
0.3 & A & 244.32410 \\
0.4 & A & 244.21300 \\
0.8 & A & 234.91650 \\
0.6 & A & 215.43982 \\
1 & A & 206.18520 \\
0.2 & A & 204.42423 \\
\hline
\end{tabular}

The COD was only measured for the wash water because the concentrations of the initial wine and final wine were extremely high and had to be diluted to samples at 1:500 to be measured properly, but this led to inaccuracies. Many of the final wine COD 
values were found to be greater than the initial wine COD values, which is not possible and gave the impression that analyzing the COD of the wine using this method was unreliable. According to the conservation of mass, the COD in the wash water should equal to the difference in COD between the initial and final wines. A decrease from the initial wine to final wine COD was noticed at times, but it did not equal the COD in the wash water. The calculated COD loss was much larger than the COD found in the wash water or showed an increase in COD after treatment. In the end, the best assumption was that the COD in the wash water was representative and proportional to the actual COD loss of the wine after treatment because of the knowledge that COD values of the wine itself was highly inaccurate and variable.

The decrease of COD through wine stabilization treatment has been noted before by both electrodialysis and cold treated wines at about 1000 ppm (Bories et al. 2011). For electrodialysis, the COD matter was mostly made up of ethanol and KHT. For the cold treated, the COD matter was mostly made up of KHT and Diachometous earth (Bories et al. 2011). In our study, the COD loss was between 200 and 300 ppm which is considerably lower than electrodialysis and cold treated wine COD loss. Most likely the COD of the wash water was an underestimate of the actual COD wash water due to not dissolving all organics and volatile organic loss during the electrolytic process. The main components in the COD matter were believed to be polyphenols, because there was no formation of KHT in this electrolytic process. The visible scaling on only the cathode suggested such a conclusion (Makhotkina and Kilmartin 2009). The fact that there was not significant positive correlation between charge and COD goes against the initial assumption when purely looking at the scaling on the plates (Figure 50 and 51). The 
higher the charge, the more scaling was observed on the electrodes. Perhaps the reason for the lack of correlation was that a lot of the precipitates may not be organic matter, but the minerals.

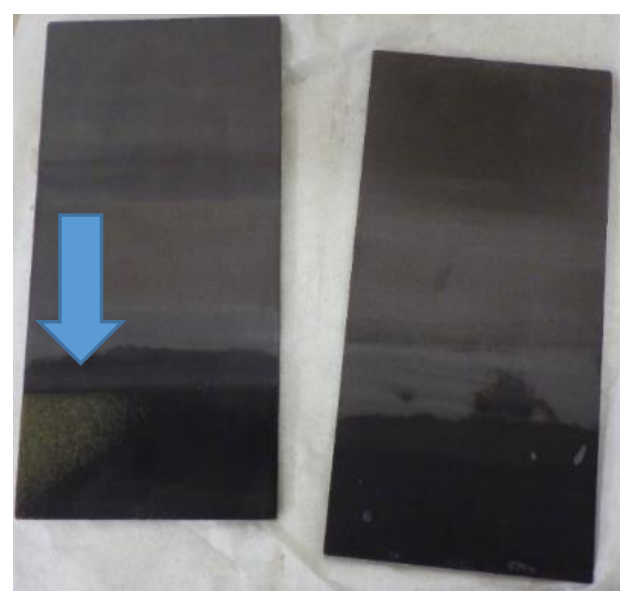

Figure 50. Scaling on cathode for $0.3 \mathrm{C}$

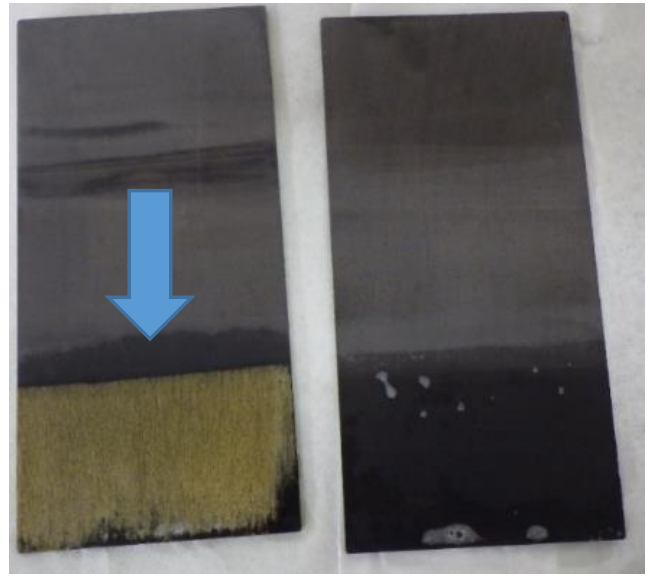

Figure 51 Scaling on cathode for $0.6 \mathrm{C}$ 


\section{CHAPTER 5}

\section{CONCLUSION}

The electrolytic method has proven under certain conditions to effectively reduce wine instability components such as tartaric acid, potassium, and calcium in Chardonnay wine. There is evidence that suggests that as charge increases so does the reduction of these instability components. The conductivity difference results pointed in a similar direction that as charge increased, so did the stability of the wine after electrolytic treatment. The cleaning process of the electrolytic method could potentially use no chemicals to clean by only using the reverse polarity water sequence.

The electrolytic method also affects some other variables of the wine. There was an increase of temperature after the electrolytic treatment and a decrease in titratable acidity, magnesium, hue, and COD. The wine most likely went through oxidative browning during the electrolytic treatment. There were no statistical significant differences for the $\mathrm{pH}$, intensity, and sodium after electrolytic treatment. The electrolytic method is a viable alternative tartrate stability process if developed further.

Now that it is seen to be possible to stabilize wine using this electrolytic method, additional research is needed to determine how the sensory characteristics compare to cold stabilized wines. Sensory is one the most important qualities besides the technical feasibilities. Whether the electrolytic process and temperature change affect the color, flavor, and aroma negatively when compared with cold stabilized wines must be studied. A cooling jacket could be used if the increase in temperature turns out to adversely affect the wine. If the quality of wine is poor, people will not likely purchase the wine. 
On the more technical side, the next step would be to improve the design of the electrolytic method by determining what type of electrodes are most effective because graphite was used purely because it was cost effective and reliable. Improvements should be done for the washing sequence to ensure clean electrodes after every run for maximum efficiency. When those parameters are determined, the optimization of process should be tackled for stability and quality, which according to this study should be around 0.8

coulombs or $\sim 13000 \frac{\mathrm{C}}{\mathrm{m}^{3}}$. Further down the road, the electrolytic system is to be designed in the shape of a pipe to streamline the stabilization and cleaning process.

Once all of the above mention steps have been completed, an economic analysis should be done on the usage of water, energy, material, and labor. If the technology is satisfactory, then the profitability or cost savings becomes extremely important in whether or not it will be commercially viable. There is promise that the electrolytic process will be feasible due to its potential of reduced processing time, water, and energy. 


\section{REFERENCES}

Agrovin. 2012. Wine tartaric stabilization system. http://www.brenn-o-kem.co.za/wpcontent/uploads/2012/11/01-Wine-tartaric-stabilization-FreeK+-System_EN2smallpdf.com-1.pdf. Accessed 2015 September 1.

Benítez JG, Macías VP, Pazo JS, Rodríguez LP. 2002. Industrial development of proton exchange for tartrate stabilization of sherry wines. Eur Food Res Technol 214:418422. doi: 10.1007/s00217-002-0504-3

Bioscience. 2008. Chemical Oxygen Demand System. Allentown: Bioscience

Bories A, Sire Y, Bouissou D, Goulesque S, Moutounet M, Bonneaud D, Lutin F. 2011. Environmental impacts of tartaric stabilisation processes for wines using electrodialysis and cold treatment. S Afr J Enol Vitic 32:174-182.

Bosso A, Salamaso D, De Faveri E, Franceschi D. 2010. The use of carboxymethylcellulose for the tartaric stabilization of white wines in comparison with other oenological additives. J of Grapevine Research 49:95-99.

Cabello-Pasini A, Macías-Carranza V, Siqueiros-Valencia A, Huerta-Díaz MÁ. 2013. Concentrations of calcium, magnesium, potassium, and sodium in wines from Mexico. Am J Enol Vitic 64:280-284. doi: 10.5344/ajev.2012.12080

Claus H, Tenzer S, Sobe M, Schlander M, Konig H, Frohilch J. 2014. Effect of carboxymethyl cellulose on tartrate salt, protein and colour stability of red wine. Aust J Grape Wine Res 20:186-193. doi: 10.1111/ajgw.12070

Coulter A. 2013. Understanding the ABCs of CMCs in stabilisation. Grapegrow Winemaker 598:72.

Das S, Li J, Hui R. 2014. Impact of Electrode Surface / Volume Ratio on Li-ion Battery Performance. COMSOL Conference; Boston, Massachusetts; 2014.

Dharmadhikari M. 2015. Wine aging. Iowa State University. http://www.extension.iastate.edu/wine/w-aging. Accessed 2009 May 20. 
Dias DA, Smith TA, Ghiggino KP, Scollary GR. 2012. The role of light, temperature and wine bottle colour on pigment enhancement in white wine. Food Chem 135:29342941. doi: 10.1016/j.foodchem.2012.07.068

Dunsford P, Boulton R. 1981. The kinetics of potassium bitartrate crystallization from table wines. I. Effect of particle size, particle surface area and agitation. Annual meeting of the American Society of Enologist; Los Angeles, California; 1980.

Fell AJ, Dykes SI, Nicolau L, Kilmartin P. 2007. Electrochemical microoxidation of red wine. Am J Enol Vitic 58:443-450.

Galpin VC. 2006. A comparison of legislation about winemaking additives and processes. [MSc thesis]. Stellenbosch, South Africa: Cape Wine Acadamy. 116 p.

Garcia-Ruiz JM, Alcantara R, Martin J. 1991. Evaluation of wine stability to potassium hydrogen tartrate precipitation. Am. J. Enol. Vitic. 46:336-340.

Georgiev SS. 2007. Optimal distance between current collecting electrodes of the solar cells. Solid State Electron 51:376-380. doi: 10.1016/j.sse.2007.01.021

Gómez Benítez J, Palacios Macías VM, Szekely Gorostiaga P, Veas Lopex R, Perex Rodriguez L. 2003. Comparison of electrodialysis and cold treatment on an industrial scale for tartrate stabilization of sherry wines. J Food Eng 58:373-378. doi: $10.1016 / \mathrm{S} 0260-8774(02) 00421-1$

Gonçalves F, Fernandes C, Cameira dos Santos P, De Pinho MN. 2003. Wine tartaric stabilization by electrodialysis and its assessment by the saturation temperature. $\mathrm{J}$ Food Eng 59:229-235. doi: 10.1016/S0260-8774(02)00462-4

Grimes C, Varghese O, Ranjan S. 2008. Light, water, hydrogen. New York: Springer.

Illand P. 2004. Chemical analysis of grapes and wine: technique and concepts. Australia: Patrick Illand Wine Promotions.

Inamuddin D, Luqman M. 2012. Ion exchange technology I. Theory and materials. New York: Springer. 
Lasanta C, Caro I, Pérez L. 2013. The influence of cation exchange treatment on the final characteristics of red wines. Food Chem 138:1072-1078. doi:

10.1016/j.foodchem.2012.10.095

Lasanta C, Gómez J. 2012. Tartrate stabilization of wines. Trends Food Sci Technol 28:52-59. doi: 10.1016/j.tifs.2012.06.005

Low LL, Neill B, Ford C, Godden J, Gishen M, Colby C. 2008. Economic evaluation of alternative technologies for tartrate stabilisation of wines. Int J Food Sci Technol 43:1202-1216. doi: 10.1111/j.1365-2621.2007.01591.x

MacDonald GM. 2010. Water, climate change, and sustainability in the southwest. Proc Natl Acad Sci 107:21256-21262. doi: 10.1073/pnas.0909651107

Makhotkina O, Kilmartin P. 2009. Uncovering the influence of antioxidants on polyphenol oxidation in wines using an electrochemical method: Cyclic voltammetry. J Electroanal Chem 633:165-174. doi:

10.1016/j.jelechem.2009.05.007

Mira H, Leite P, Ricardo-Da-Silva JM, Curvelo-Garcia AS. 2006. Use of ion exchange resins for tartrate wine stabilization. J. Int. Sci. Vigne Vin 40:223-246.

O'Brien V. 2014. Capitalising on tartrate stabilisation. Grapegrow Winemaker 577:6164.

OIV. 2009. Compendium of international methods of analysis-OIV chromatic characteristics method. OIV-MA-AS2-07B.

OIV. 2013. Analysis of mineral elements in wines using ICP-AES (inductively coupled plasma / atomic emission spectrometry). OIV-MA-AS322-13.

Oliveira CM, Ferreira ACS, De Freitas V, Silva AMS. 2011. Oxidation mechanisms occurring in wines. Food Res Int 44:1115-1126. doi: 10.1016/j.foodres.2011.03.050

Pacific Gas and Electric Company: Wine stabilization through electrodialysis. [Internet] PG\&E; 2007 [Accessed 2010 April 20]. Available from: http://www.baseco.com/Publications/ETCC Report - Wine Stabilization through 
Electrodialysis.pdf.

Parr W V, Geoffrey White K, Heatherbell D. 2003. The nose knows: influence of colour on perception of wine aroma. J Wine Res 14:79-101. doi:

$10.1080 / 09571260410001677969$

Ribereau-Gayon P, Glories Y, Maujean A, Dubourdieu D. 2006. Handbook of enology, 2nd edition. Chichester: John Wiley \& Sons.

Rodriguez-Clemente R, Correa-Gorospe I, De Castro JJ. 1988. A new method for the stabilization of wines with respect to the potassium bitartrate precipitation. Am J Enol Vitic 41:16-20.

Roget W. 2012. Benchmarking a continuous tartrate stabilisation system. Grapegrow Winemaker 585:106-108.

Santos PC Dos, Gonçalves F, De Pinho MN. 2002. Optimisation of the method for determination of the temperature of saturation in wines. Anal Chim Acta 458:257261.

Soares P, Geraldes V, Fernandes C, Santos P, Pinho M. 2009. Wine tartaric stabilization by electrodialysis: Prediction of required deionization degree. Am J Enol Vitic 60:183-188.

Song Y, Song Y, Xu J (2012) Electrochemical determination of tartaric acid at nano gold/nano carbon modified glassy carbon electrode. Russ J Phys Chem A 86:14581464. doi: 10.1134/S0036024412070345

STARS: ED/STARS Advantages.[Internet]. Oenodia; 2011 [Accessed 2014 September 1]. Available from: http://wineindustrynetwork.com/uploads/service/YTNwckxlaDbmWxmI3Re1 ynWB OiEMmM.pdf.

Tanaka H and Tatsuya H, inventors; 2008 May 22. Cooling water circulation apparatus and method of removing scale from cooling water circulation apparatus. U.S. patent $0,115,925$.

Walker T, Morris J, Threlfall R, Main G. 2004. Quality, sensory and cost comparison for $\mathrm{pH}$ reduction of syrah wine using ion exhcnage or tartaric acid. J Food Qual 27:483496. 
Zoecklein B, Fugelsang K, Gump B, Nury F. 1990. Production wine analysis. New York: Chapman \& Hall. 


\section{APPENDIX}

Table 17. Tukey comparison of treatments for mean differences in temperature.

\begin{tabular}{llr}
\hline Treatment & \multicolumn{2}{c}{ DLeast Sq Mean } \\
\hline 12 & $\mathrm{~A}$ & 10.000000 \\
8 & $\mathrm{~A}$ & 9.083333 \\
11 & $\mathrm{~A}$ & 7.916667 \\
10 & $\mathrm{~A}$ & 7.416667 \\
4 & $\mathrm{~A}$ & 7.333333 \\
7 & $\mathrm{~A}$ & 7.166667 \\
3 & $\mathrm{~A}$ & 7.000000 \\
9 & $\mathrm{~A}$ & 6.250000 \\
6 & $\mathrm{~A}$ & 5.500000 \\
2 & $\mathrm{~A}$ & 5.333333 \\
5 & $\mathrm{~A}$ & 5.166667 \\
1 & $\mathrm{~A}$ & 5.042329 \\
\hline
\end{tabular}

Table 18. Tukey comparison of treatments for mean differences in tartaric acid concentration.

\begin{tabular}{|c|c|c|c|c|c|c|}
\hline Treatment & & & & & & $\Delta$ Least Sq Mean \\
\hline 12 & $A$ & & & & & 1.0666667 \\
\hline 11 & $A$ & B & & & & 0.8000000 \\
\hline 8 & $A$ & B & C & & & 0.7666667 \\
\hline 10 & $A$ & B & C & $D$ & & 0.7333333 \\
\hline 13 & & B & C & D & & 0.6777779 \\
\hline 7 & & B & $\mathrm{C}$ & D & $E$ & 0.5333333 \\
\hline 9 & & B & C & D & $E$ & 0.4666667 \\
\hline 6 & & B & C & D & $E$ & 0.4000000 \\
\hline 4 & & & C & D & $E$ & 0.3333333 \\
\hline 3 & & & & D & $E$ & 0.3000000 \\
\hline 5 & & & & & $E$ & 0.2833333 \\
\hline 2 & & & & & $E$ & 0.2666667 \\
\hline 1 & & & & & $E$ & 0.0733312 \\
\hline
\end{tabular}

Table 19. Tukey comparison of treatments for least square mean differences in titratable acidity.

\begin{tabular}{llr}
\hline Treatment & D Least Sq Mean \\
\hline 10 & $\mathrm{~A}$ & 1.2703870 \\
12 & $\mathrm{~A}$ & 1.1112604 \\
11 & $\mathrm{~A}$ & 1.0332000 \\
7 & $\mathrm{~A}$ & 0.8274222 \\
8 & $\mathrm{~A}$ & 0.8140889 \\
3 & $\mathrm{~A}$ & 0.7036877 \\
9 & $\mathrm{~A}$ & 0.6815111 \\
13 & $\mathrm{~A}$ & 0.6618418 \\
6 & $\mathrm{~A}$ & 0.6422382 \\
5 & $\mathrm{~A}$ & 0.5705333 \\
4 & $\mathrm{~A}$ & 0.5444889 \\
2 & $\mathrm{~A}$ & 0.5185185 \\
1 & $\mathrm{~A}$ & 0.1961315 \\
\hline
\end{tabular}

Table 20. Tukey comparison of treatments for mean differences in $\mathbf{p H}$.

\begin{tabular}{llr}
\hline Treatment & DLeast Sq Mean \\
\hline 12 & A & 0.0600000 \\
1 & A & 0.0493687 \\
8 & A & 0.0466667 \\
7 & A & 0.0400000 \\
4 & A & 0.0400000 \\
3 & A & 0.0333333 \\
2 & A & 0.0333333 \\
13 & A & 0.0205556 \\
11 & A & 0.0133333 \\
5 & A & -0.0033333 \\
6 & A & -0.0066667 \\
9 & A & -0.0166667 \\
10 & A & -0.02333333 \\
\hline
\end{tabular}

Table 21. Tukey comparison of treatments for mean differences in hue

\begin{tabular}{llr}
\hline Treatment & & Least Sq Mean \\
\hline 10 & $\mathrm{~A}$ & 2.707352 \\
8 & $\mathrm{~A}$ & 2.670618 \\
12 & $\mathrm{~A}$ & 2.349680 \\
7 & $\mathrm{~A}$ & 2.055139 \\
6 & $\mathrm{~A}$ & 1.828137 \\
4 & $\mathrm{~A}$ & 1.792399 \\
11 & $\mathrm{~A}$ & 1.784106 \\
2 & $\mathrm{~A}$ & 1.679835 \\
3 & $\mathrm{~A}$ & 1.545228 \\
5 & $\mathrm{~A}$ & 1.474256 \\
9 & $\mathrm{~A}$ & 1.331940 \\
1 & $\mathrm{~A}$ & 1.242857 \\
13 & $\mathrm{~B}$ & -1.180463 \\
\hline
\end{tabular}

Table 22. Tukey comparison of treatments for mean differences in intensity

\begin{tabular}{llr}
\hline Treatment & \multicolumn{1}{c}{$\boldsymbol{\Delta L e a s t ~ S q ~ M e a n ~}$} \\
\hline 13 & $\mathrm{~A}$ & 0.0831387 \\
5 & $\mathrm{~A}$ & 0.0143333 \\
6 & $\mathrm{~A}$ & 0.0080000 \\
9 & $\mathrm{~A}$ & 0.0036667 \\
10 & $\mathrm{~A}$ & -0.0080000 \\
12 & $\mathrm{~A}$ & -0.0130000 \\
2 & $\mathrm{~A}$ & -0.0153333 \\
8 & $\mathrm{~A}$ & -0.0180000 \\
1 & $\mathrm{~A}$ & -0.0195000 \\
7 & $\mathrm{~A}$ & -0.0200000 \\
4 & $\mathrm{~A}$ & -0.0283333 \\
3 & $\mathrm{~A}$ & -0.0293333 \\
11 & $\mathrm{~A}$ & -0.0460000 \\
\hline
\end{tabular}


Table 23. Tukey comparison of treatments for mean differences in potassium

\begin{tabular}{llr}
\hline Treatment & & DLeast Sq Mean \\
\hline 13 & A & 27.200666 \\
12 & A & 24.516667 \\
8 & A & 22.341667 \\
11 & A & 22.150000 \\
10 & A & 19.508333 \\
4 & A & 16.175000 \\
7 & A & 11.716667 \\
2 & A & 5.116667 \\
5 & A & 4.975000 \\
1 & A & 3.109735 \\
9 & A & 2.833333 \\
6 & A & 1.941667 \\
3 & A & 1.366667 \\
\hline
\end{tabular}

Table 24. Tukey comparison of treatments for mean difference in calcium

\begin{tabular}{|c|c|c|c|c|}
\hline Treatment & & & & $\Delta$ Least Sq Mean \\
\hline 12 & $A$ & & & 9.350000 \\
\hline 8 & $A$ & B & & 6.175000 \\
\hline 11 & $A$ & B & & 5.866667 \\
\hline 7 & $A$ & B & & 4.858333 \\
\hline 10 & $A$ & B & & 4.141667 \\
\hline 4 & & B & & 3.175000 \\
\hline 3 & & B & & 2.533333 \\
\hline 9 & & B & & 1.583333 \\
\hline 2 & & B & 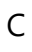 & 1.575000 \\
\hline 6 & & B & $C$ & 1.275000 \\
\hline 1 & & B & & 0.713063 \\
\hline 5 & & B & 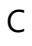 & 0.625000 \\
\hline 13 & & & C & -2.704861 \\
\hline
\end{tabular}

Table 25. Tukey comparison of treatments for mean differences in magnesium

\begin{tabular}{|c|c|c|c|c|c|}
\hline Treatment & & & & & $\Delta$ Least Sq Mean \\
\hline 12 & $A$ & & & & 23.23333 \\
\hline 8 & A & B & & & 15.14167 \\
\hline 11 & $A$ & B & C & & 14.60833 \\
\hline 10 & A & B & C & & 11.14167 \\
\hline 7 & & B & C & & 9.40000 \\
\hline 4 & & B & C & D & 7.59167 \\
\hline 3 & & B & C & D & 5.79167 \\
\hline 2 & & B & C & D & 4.39167 \\
\hline 1 & & B & C & D & 3.57782 \\
\hline 5 & & & C & D & 2.16250 \\
\hline 9 & & & C & $\mathrm{D}$ & 2.14167 \\
\hline 6 & & & C & $\mathrm{D}$ & 2.12500 \\
\hline 13 & & & & $\mathrm{D}$ & -1.89861 \\
\hline
\end{tabular}

Table 26. Tukey comparison of treatments for mean differences in sodium

\begin{tabular}{llrr}
\hline Treatment & & & $\Delta$ Least Sq Mean \\
\hline 3 & A & & 0.891667 \\
6 & A & B & 0.716667 \\
11 & A & B & 0.691667 \\
2 & A & B & 0.650000 \\
4 & A & B & 0.633333 \\
8 & A & B & 0.616667 \\
12 & A & B & 0.491667 \\
10 & A & B & 0.433333 \\
7 & A & B & 0.300000 \\
1 & A & B & 0.269131 \\
9 & A & B & 0.233333 \\
5 & A & B & 0.195833 \\
13 & & B & -2.371542 \\
\hline
\end{tabular}

